SERVIÇO DE PÓS-GRADUAÇÃO DO ICMC-USP

Data de Depósito: $20.02 \cdot 2003$

Assinatura: L n a Paula lampaiór refone

\title{
Estimação não-paramétrica da taxa de falha acumulada de um processo pontual
}

Fabrizio Teixeira Mendes"

Orientador: Prof. Dr. Dorival Leão P. Júnior

Dissertação apresentada ao Instituto de Ciências Matemáticas e de Computação - ICMC-USP, como parte dos requisitos para obtenção do título de Mestre em Ciências de Computação e Matemática Computacional.

USP - São Carlos

Fevereiro/2003

* Este trabalho contou com o apoio financeiro da CAPES. 
A Comissão Julgadora:

Prof. Dr. Dorival Leão Pinto Junior

Prof. Dr. José Galvão Leite

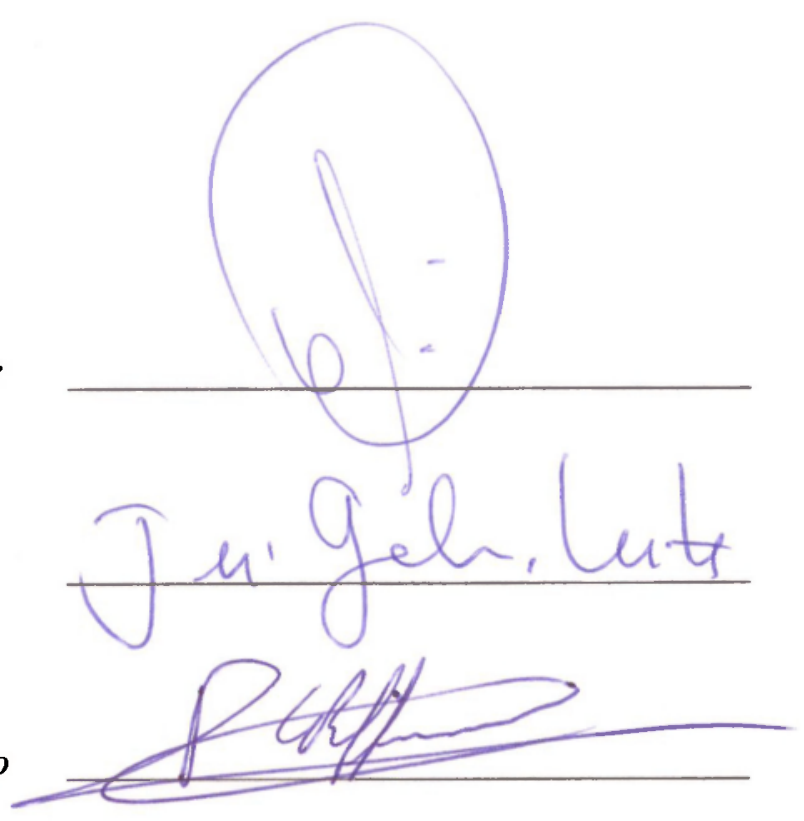


À minha família,

minha namorada Roberta.

.. e a todos os meus amigos, 


\section{Agradecimentos}

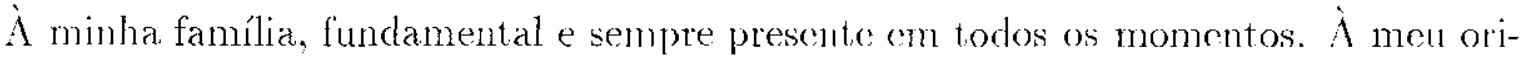
entador Dorival hào pela oportuniclade, amizade, por suld dedicaço e pelo seu empenho na realizaça desse trabalho. Aos professores Jorge Alberto Achear e Joś́ Galvâo Leite participantes da banca de qualificação. Aos professores Marinho Gomes de Audrade e Reiko Aoki pelas disciplinas ministradas e pela comstante ajuda que nos têm dado. $\grave{A}$ meu ex-orientador de iniciaçào científica Sérgio Luis Zaıı. À alguns cx-professores mens

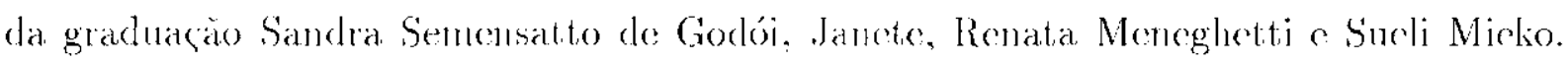
A mens ex-companheiros de graduaçầo è aniga Lívia que já năo está majs aqui. Aos funcionários da biblioteca pelo anxilio que nos têm dado. $\lambda$ todos os funcionários do IC.MC. A todo o pessoal legal do laboratório da Estatística (em ordem alfabética), Aline, Flávia, (iecirlei, Paulo, Ricardo, pela ajuda e pela amizade e a todos os amigos que, de uma forma ou outra contribuiram com um perdacinho para essa concquista. Desculpent-me se por acaso en tiver esçuecido alguén. 


\section{Resumo}

Vários autores ten construído estimadores de Bayes nâo-paramétricos para a funçào de distribuição acumulada. A distribuiçào à priori tem, por exemplo, sido processos de Dirichlet, processos neutral to the right. Yeste trabalho nós estudamos o problema de achar estimadores de Bayes não-paramétricos para a taxa de fallha acumulada de um processo pontuaj baseado no modelo de intensidade multiplicativo de Aalen. Desta forma nós consideramos una classe conjugada de processos de Lévy chamados de processos beta caprescrutamos fómulas para obter um processo posterior. O estimador de Bayes é comparado com dois outros estimadores năo-paramétricos, Kaplan-Meier e Nelson-Aalen e un estinador paramétrico a laxa de falha acumulada de una distribuiçào Weibull. 


\begin{abstract}
Several authors have constructed nonparametric Baycs estimators for a cumulative distributijon function. The prior distribution have, for example, been Dirichlet processes, neutral to the right processes. In this work we studied the problem of finding nonparametric Bayes estimator to cumnlative rate function of the a point processes based in the Aalen's multiplicative intensity model. This form we considered a conjugate class of Lévy processes called beta processes and prescited formulas for obtaining a posterior processes. The Bayes sstimator is compared with two nomparametric estimator, Kaplan-Meier and Nelson-Aalen and one parametric estimator, the cumulative rate function of the Weibull distribution.
\end{abstract}




\section{Sumário}

1 Introdução 1

1.1 Estimadores Empíricos . . . . . . . . . . . . . . . 2

1.1 .1 Estimador de Kaplan- Yeier . . . . . . . . . . . . . . . . . 3

1.1.2 Estimador do Nelson-Aalon . . . . . . . . . . . . . . 5

1.1.3 Análise dos Dados . . . . . . . . . . . . . . . . . . 7

1.2 A cstimaçàio nào-paramétrica . . . . . . . . . . . . . 11

1.2 .1 Modelo Fstalístico . . . . . . . . . . . . . . . . 15

1.2 .2 Estimador do Bayes . . . . . . . . . . . . . . . . . . . 17

2 O Processo Pontual 23

2.1 () Espaco de Trajetórias . . . . . . . . . . . . . . . . . . . 23

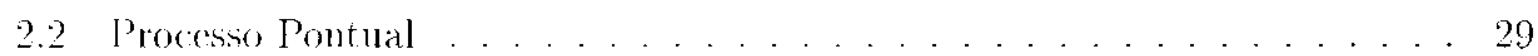

2.3 Decomposição de Doob-Neyer . . . . . . . . . . . . . . . . . 34

2.4 Taxa de Falha do Processo Pontual . . . . . . . . . . . . . . . 40

2.5 Representação do Processo Pontual . . . . . . . . . . . . . . . . 42

3 Processos de Lévy Puro Salto $\quad 45$

3.1 Introdncão . . . . . . . . . . . . . . . . . . . . . 45

3.2 Processos de Lévy Puro Salto . . . . . . . . . . . . . . . . . . 45

3.3 Representação do Processo de Lévy . . . . . . . . . . . . . . . . 52

3.4 Processos Beta . . . . . . . . . . . . . . . . . 54

4 Inferência Bayesiana $\quad 57$

4.1 Introdução . . . . . . . . . . . . . . . . . . . 57

4.2 Estimação Bayesiana Vàs-Paramétrica . . . . . . . . . . . 58

4.3 Estimador Baycsiano Và-Paramétrico para a Taxa de Falha Acumulada . 60 
5 Considerações Finais $\quad 69$

j.1 Conclusoes. . . . . . . . . . . . . . . . . . . . . . 69

5.2 Propostas Finturas . . . . . . . . . . . . . . . . . . 73

6 Apêndice $\quad 75$

6.1 Espaço de Cantor . . . . . . . . . . . . . . . . . . . . 75

6.2 Alguns Resultados titilizados . . . . . . . . . . . . . . . . . . . 77

$\begin{array}{ll}\text { Referências Bibliográficas } & 78\end{array}$ 


\section{Lista de Figuras}

1.1 Gráfico das estimativas da taxa de falha via Nelson-Aalen e Weibull . . . . 9

1.2 Gráfico das estimativas da funçâo de confiabilidade . . . . . . . . . . 11

1.3 Gráfico das estimativas da taxa de falha acumulada . . . . . . . . . . . 11

1.4 Martingale para a taxal acumulada de uma Weibull . . . . . . . . . 14

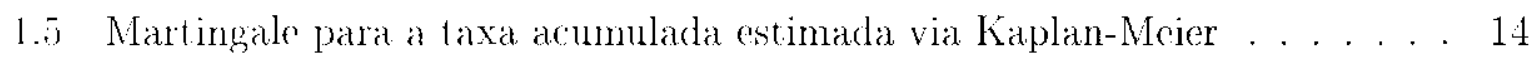

1.6 Gráficos da Taxa de Falha acumulada estimada via Kaplan-Meier, Nelson-

Aalen. Weibull e Bayesiano Empírico . . . . . . . . . . . . . . . 21

4.1 Taxa de Falha Acumulada . . . . . . . . . . . . . . . . . 66

4.2 Funçăo de Confiabilidade . . . . . . . . . . . . . . . . 66

4.3 Taxa de Falha . . . . . . . . . . . . . . . . . . . . . 67

5.1 Taxa de Falha Acumulada . . . . . . . . . . . . . . . . . . 70

5.2 Taxa de Falha . . . . . . . . . . . . . . . . . 73 


\section{Lista de Tabelas}

1.1 Tempos de Vida dos Freios em Teste . . . . . . . . . . . . . . . 2

1.2 Fstimativas da variancia da taxa de falha acumulada via Kaplan-Meier . . 5

1.3 Estimativas da variancia da taxa de falha acumulada via Nelson-Aalen . . 6

1.4 Estimativas da laxa de falha via Kaplan-Meier, Nelson-Aalen e Weibull . . 8

1.j Estimativas da laxa de falha acumulada . . . . . . . . . . . . 10

4.1 Fstimativas da variancia da taxa de falha acumulada via estimador Bayesiano 64

4.2 Estimativas para a taxa de falha, taxa de falha acumulada e função de confiabilidade baseadas no estimador Bayesiano não-paramétrico . . . . . . 60

J.1 Estimativas da variância da taxa de falha acumulada via Kaplan-Meier . . 71

5.2 Estimativas da variância da taxa de falha acumulada via Nelson-Aalen . . 71

5.3 Estimativas da variância da taxa de falha acurrulada via estimador Bayesiano 72 


\section{Capítulo 1}

\section{Introdução}

() estudo da inferência nāo paramétrica permite uma análise mais flexível e geral dos dados, pois ela năo sé prende a nenbum tipo de distribuição paramétrica. A estimação nãoparamétrica possui boas propricdades mesmo para pequenas amostras, essa flexibilidade no entanto, é custosa pois devemos trabalhar sobre um espaço de funçōes. Quando fazemos a análise estatísticia atravós de modelos obtidos por farnílias paramétricas de distribuições, estamos restringindo um espaço de dimensiào infinita à um de dimensão finita cujo objetivo ć geralmente estimar um número finito de parâmetros.

Neste trabalho, consideramos $X_{1}, \ldots, X_{n}$ uma sequiência de variáveis alcatórias positivas independentes e identicamente distribuídas com função distribuição acumulada $F$. Ao denotarmos por $T_{1}, \ldots, T_{n}$ as estatísticas de ordern da amostra aleatória $\left(X_{1}, \ldots, X_{n}\right)$, podemos associar um processo pontual por

$$
N_{t}=\sum_{i-1}^{n} I_{\left\{X_{\imath}<t\right\}}=\sum_{i=1}^{n} I_{\left\{T_{i} \leq \imath\right\}} .
$$

Desde que $N_{t}$ é um processo näo-decrescente, concluímos que $N_{t}$ ó um submartingale. Utilizando a decomposição de Doob-Mcyer, existe um único processo estocástico $\Lambda$ (previsível) taal (1ue

$$
M_{t}=: N_{t}-\Lambda_{t}=N_{t}-\int_{0}^{t} Y_{s} d A_{s}
$$

co um martingale, onde $Y_{s}=n-N_{s-}$ e $A$ é a taxa de falha acumulada. Além disso, a distribuiçäo de probabilidade definida por $N_{t}$ ć unicamente determinada pelo processo $\Lambda_{t}$. Desde que $Y_{s}$ ć obtido diretamente da amostra, vamos analisar alguns estimadores empíricos e utilizar uma abordagem Bayesiana não-paramétrica para estimar a taxa de falha acumulada $A_{t}$. Para estudtá-la utilizando o enfoque Bayesiano não-paramétrico vamos 
definir uma probabilidade inicial sobre o espaço das funções taxas de falha acumulada e calcular o estimador de Bayes através da distribuição à posteriori.

Para ilustrar a aplicação das técnicas descritas acima, vamos considerar um conjunto de tempos de falha obtidos pela EMBRAER. Com objetivo de estudar a performance dos freios do avião ER.J 145, a EMBRAER realizou um estudo com 30 aviōes. Um freio novo (sem defeito) foi colocado em cada aeronave e acompanhado até a sua falha. O tempo de falha (em horas) do freio de cada avião encontra-se abaixo:

\begin{tabular}{|c|c|c|c|c|c|c|c|c|c|}
\hline 19 & 43 & 44 & 148 & 169 & 171 & 205 & 232 & 248 & 250 \\
\hline 263 & 271 & 282 & 290 & 347 & 349 & 398 & 477 & 514 & 595 \\
\hline 603 & 662 & 700 & 701 & 706 & 709 & 710 & 763 & 777 & 869 \\
\hline
\end{tabular}

Tabela 1.1: Tempos de Vida dos Freios em Teste

O objetivo da EMBRAER consiste em estimar a probabilidade de um freio falhar em cada instante de tempo. A modelagem que faremos dos dados será feita scm a presença de censuras, pois esses freios gastam-se muito rapidamente e todos os tempos de falha são observados. Também em nossa análise não iremos considerar a presença de falhas coincidentes(empates).

\subsection{Estimadores Empíricos}

Considere $(\Omega, \Im, P)$ um espaço de probabilidade e $T \geq 0$ uma variável aleatória arbitrária com densidade $f(t)$ e função distribuiçăo $F(t)$. Em confiabilidade temos interesse no estudo das funções de confiabilidade

$$
S(t)=P[T>t]=1-F(t)
$$

taxa de falha

$$
\lambda(t)=\lim _{\Delta t \rightarrow 0} \frac{P[t \leq T<t+\Delta t \mid T>t]}{\Delta t} .
$$

e taxa de falha acumulada

$$
A(t)=\int_{0}^{t} \lambda(s) d s
$$

Desde que $T$ possui densidade temos

$$
\lambda(t)=\lim _{\Delta t \rightarrow 0} \frac{P[t \leq T<t+\Delta t \mid T>t]}{\Delta t}=\lim _{\Delta t \rightarrow 0} \frac{1}{\Delta t} \frac{P[t \leq T<t+\Delta t]}{P(T>t]}=\frac{f(t)}{S(t)}
$$


Assim, a taxa de falha acumulada de Tica da seguinte forma,

$$
\begin{aligned}
A(t) & =\int_{0}^{t} \lambda(s) d s=\int_{0}^{t} \frac{f(s)}{S(s)} d s \\
& =-\int_{0}^{t} \frac{d S(s)}{S(s)}=-\ln \{S(t)\}
\end{aligned}
$$

A função de confiabilidade representa a probabilidade do freio sobreviver mais que o tempo t e a taxa de falha especifica a razão de falha instantânea no tempo $T=t$ darlo que a falha não ocorreu até o tempo t. Para $S(t)$ e $A(t)$ existem estimadores não-paramétricos, () produto limite ou Kaplan-Meier e o estimador de Nelson-Aalen. A fórmula 1.1 foi usada por Nelson(1972) para obter um estimador năo paramétrico para a taxa de falha acumulada. Outra expressäo que derivamos desta fórmula é

$$
S(t)=\exp \{-A(t)\}
$$

\subsubsection{Estimador de Kaplan-Meier}

O estimador de Kaplan-Meier, proposto em 1958, também chamado de produto-limite, é um dos estimadores empíricos mais utilizados em análise de confiabilidade. Ele é uma função escada, constante entre dois tempos de falha consecutivos. Esta função dá umn salto no instante da falha ou morte. Assim, dados os tempos de falha $0=t_{0}<t_{1}<\ldots<t_{n}$, podemos encontrar o estimador de Kaplan-Meier da função de confiabilidado da seguinte forma. Considere a funçăo de confiabilidade até o tempo $t_{k}$

$$
S\left(t_{k}\right)=P\left[T>t_{k}\right]
$$

Vanos mostrar que a função de confiabilidade acima pode ser escrita na forma

$$
S\left(t_{k}\right)=\prod_{j=1}^{k}\left(1-P i t_{j} .\left|\leq T<t_{j}\right| t_{j-1} \mid\right)
$$

para todo $k=1, \cdots, n$.

Para isto, vamos verificar que o resultado é válido pelo método de indução. Para $k=1$ temos

$$
S\left(t_{1}\right)=P\left[T>t_{1}\right]-1-P\left[T \leq t_{1}\right]=1-P\left[t_{0} \leq T<t_{1}\right] .
$$

Suponha que o resultado é válido para $k-1$, vamos mostrar que ele é também válido 
para $k$. Para isto, temos que

$$
\begin{aligned}
S\left(t_{k}\right) & =P\left[T>t_{k}\right]=1-P\left[T \leq t_{k}\right]=1-\left(P\left[\left\{T \leq t_{k-1}\right\} \cup\left\{t_{k} 1 \leq T<t_{k}\right\}\right]\right) \\
& =1-\left(P\left[T \leq t_{k-1}\right]+P\left[t_{k-1} \leq T<t_{k}\right]\right)=1-P\left[T \leq t_{k-1}\right]-P\left[t_{k-1} \leq T<t_{k}\right] \\
& =S\left(t_{k-1}\right)-P\left[T>t_{k-1}\right] P\left[t_{k-1} \leq T<t_{k} \mid T>t_{k-1}\right] \\
& =S\left(t_{k-1}\right)-S\left(t_{k-1}\right) P\left[t_{k-1} \leq T<t_{k} \mid T>t_{k-1}\right] \\
& =S\left(t_{k-1}\right)\left(1-P\left[t_{k-1} \leq T<t_{k} \mid T>t_{k-1}\right]\right) \\
& =\prod_{j=1}^{k}\left(1-P\left[t_{j-1} \leq T<t_{j} \mid t_{j-1}\right]\right) .
\end{aligned}
$$

Portanto o resultado é válido para $k-1, \cdots, n$. Com isso, urn estimador empírico para a função de confiabilidade pode ser dado por

$$
\widehat{S}_{K M}(t)=\prod_{k: t_{k} \leq t}\left(1-\widehat{q}_{k}\right)
$$

onde os $q_{k}$ são dados por

$$
q_{k}=P\left[t_{k-1}<T \leq t_{k} \mid T>t_{k-1}\right]-\frac{P\left[t_{k-1}<T \leq t_{k}\right]}{P\left[T>t_{k-1}\right]}
$$

e podem ser estimados empiricamente da seguinte forma

$$
\widehat{q_{k}}=\frac{d_{k}}{n_{k}}
$$

onde $n_{k}$ é o número de peças que não falharam em $t_{k}$ e $d_{k}$ é o número de falhas ocorridas em $t_{k}$, que vamos considerar igual a 1 (pois admitimos que não há falhas coincidentes). O estimador $\widehat{q}_{k}$ é considerado um estimador empírico para a taxa de falha $\lambda$ no intervalo $\left[\ell_{k-1}, t_{k}\right)$. Assim, o estimador produto-limite ou Kaplan-Mejer para a função de confiabilidade é dado por

$$
\widehat{S}_{K M}(t)=\prod_{k: t_{k} \leq t}\left(1-\widehat{q}_{k}\right)=\prod_{k: t_{k} \leq t}\left(1-\frac{1}{n_{k}}\right)
$$

e um estimador para sua variância por

$$
\widehat{V}\left[\widehat{S}_{K M}(t)\right]=\left[\widehat{S}_{K M}(t)\right]^{2} \sum_{k: t_{k} \leq t} \frac{\widehat{q}_{k}}{n_{k}\left(1-\widehat{q}_{k}\right)} .
$$

Também, podemos usar o estimador de Kaplan-Meier para estimar a taxa de falha acumulada através da aplicaçäo da Equaçăo 1.1,

$$
\widehat{A}_{K M}=-\ln \left\{\widehat{S}_{K M}(t)\right\} .
$$


O estimador da variancia desse estimador é obtido via fórmula de Greenwood e é dado por

$$
\widehat{V}\left[\widehat{A}_{K M}(t)\right]=\sum_{i: t_{i} \leq t} \frac{1}{n_{i}\left(n_{i}-1\right)} .
$$

Para mais detalhes ver Kaplan e Meier [1958, pg. 477] e Ansell e Phillips [1994, pg. 38].

A tabela 5.1 apresenta as estimativas da variância do estimador da taxa de falha acumulada via estimador de Kaplan-Meicr.

\begin{tabular}{|c|c|c|c|}
\hline tempos & variancia & tempos & varianncia \\
\hline \hline ternpos & variancià & tempos & variancia \\
\hline 19 & 0.001149 & 349 & 0.038095 \\
\hline 43 & 0.002381 & 398 & 0.043590 \\
\hline 44 & 0.0003704 & 477 & 0.050000 \\
\hline 148 & 0.005128 & 514 & 0.057576 \\
\hline 169 & 0.0066667 & 595 & 0.0666667 \\
\hline 171 & 0.008333 & 603 & 0.077778 \\
\hline 205 & 0.010145 & 662 & 0.091667 \\
\hline 232 & 0.012121 & 710 & 0.109524 \\
\hline 248 & 0.014286 & 701 & 0.133333 \\
\hline 250 & 0.016667 & 706 & 0.166667 \\
\hline 263 & 0.019298 & 709 & 0.216667 \\
\hline 271 & 0.022222 & 710 & 0.300000 \\
\hline 282 & 0.025490 & 763 & 0.466667 \\
\hline 290 & 0.0129167 & 777 & 0.966667 \\
\hline 347 & 0.033333 & 869 & $*$ \\
\hline
\end{tabular}

Tabcla 1.2: Estimativas da variância da taxa de falha acumulada via Kaplan-Meier

\subsubsection{Estimador de Nelson-Aalen}

Nesta seça vamos apresentar o estimador proposto por Nelson (1972) e estudado por Aalen (1978) para a função taxa de falha acumulada. Como visto anteriormente, o estimador para a taxa de falha $\lambda(t)$ é dado por:

$$
\widehat{\lambda}(t)=\widehat{q}_{i}=\frac{1}{r_{i}}, t_{i-1} \leq t<t_{i}
$$

Assim, um estimador empírico para a taxa de falha acumulada pode ser obtida na forma,

$$
\widehat{A}(t)=\sum_{i: t_{i} \leq t}\left(\frac{1}{n_{i}}\right)
$$

e um estimador para a função de confiabilidade por [Equação 1.1],

$$
\widehat{S}_{N A}(t)=\exp \{-\widehat{A}(t)\} \text {. }
$$


O estimador da taxa de falha acumulada de Velson-Aalen pode ser escrito na forma de uma integral de Stieltjes,

$$
\begin{aligned}
\widehat{A}(t) & =\sum_{k: t_{k} \leq t}\left(\frac{d_{k}}{n_{k}}\right)=\sum_{k: t_{k} \leq t}\left(\frac{1}{n_{k}}\right)=\sum_{k}\left(\frac{N_{t_{k}}-N_{t_{k-}}}{\sum_{i} I_{\left\{t_{i}>t_{k}\right\}}}\right)= \\
& =\sum_{k}\left(\frac{N_{t_{k}}-N_{t_{k-}}}{Y\left(t_{k}\right)}\right)=\int_{0}^{t} \frac{I_{\{Y(s)>0\}}}{Y(s)} d N_{s},
\end{aligned}
$$

onde $N_{t}=\sum_{k} I_{\left\{t_{k}<\imath\right\}}$ é uma função de contagem, que conta o número de falhas até o tempo $t, N_{t-}=\lim _{s \uparrow t} N_{s}, I_{\{.\}}$é uma função indicadora e $Y(t)=\sum_{i} I_{\left\{t_{2}<t\right\}}=n-N_{t--}$. No caso de ocorrer $Y(s)=0$, teremos $\widehat{A}(t)-0$.

A variância do estimador de Nelson-Aalen $\widehat{A}(t)$ é estimada mais adiante na seção 1.2 via decomposição de Doob-Meyer [ver Equação 1.8]. Em estimador para a variància do estimador de Nelson-Aalen é dado por:

$$
\widehat{\operatorname{Var}}[\widehat{A}(t)]=\int_{0}^{t} \frac{J_{s} d N_{s}}{Y_{s}^{2}}=\sum_{k: t_{k} \leq t} \frac{1}{n_{k}^{2}}
$$

As estimativas da variância do estimador de Nelson-Aalen estão na Tabela 1.3

\begin{tabular}{|c|c|c|c|}
\hline tempos & variäncia & tempos & variância \\
\hline \hline 19 & 0.00111 & 349 & 0.03615 \\
\hline 43 & 0.00230 & 398 & 0.04126 \\
\hline 44 & 0.00358 & 477 & 0.04717 \\
\hline 148 & 0.00495 & 514 & 0.05412 \\
\hline 169 & 0.006443 & 595 & 0.06238 \\
\hline 171 & 0.00803 & 603 & 0.07238 \\
\hline 205 & 0.00976 & 662 & 0.08473 \\
\hline 232 & 0.01165 & 700 & 0.10035 \\
\hline 248 & 0.01372 & 701 & 0.12076 \\
\hline 250 & 0.01599 & 706 & 0.14454 \\
\hline 2633 & 0.018 .49 & 709 & 0.18854 \\
\hline 271 & 0.02126 & 710 & 0.25104 \\
\hline 282 & 0.02434 & 76.3 & 0.36215 \\
\hline 290 & 0.02780 & 777 & 0.61215 \\
\hline 347 & 0.03171 & 869 & 1.61215 \\
\hline
\end{tabular}

Tabela 1.3: Estimativas da variância da taxa de falha acumulada via Nelson- $\mathrm{Aalen}$ 


\subsubsection{Análise dos Dados}

Nesta seçäo, vamos aplicar os estimadores de Kaplan-Meier e Nelson-Aalen ao conjunto de dados fornecidos pela EMBRAER. A esse conjunto de dados verificamos, através do papel de probabilidade, que uma distribuição de Weibull com função densidade de probabilidade dada por

$$
f(t)=\frac{b}{a^{b}} t^{b-1} \exp \left[-\left(\frac{t}{a}\right)^{b}\right], a, b>0 .
$$

fica bem ajustada. Suas taxa de falha e taxa de falha acumulada são dadas, respectivamente por:

$$
\begin{gathered}
h(t)=\frac{b}{a^{b}} t^{b-1} \\
H(t)=\left(\frac{t}{a}\right)^{b} .
\end{gathered}
$$

As estimativas de máxima verossimilhança obtidas para os parâmetros dessa distribuição foram, parâmetro de escala $\widehat{a}=462,13$ e parámetro de forma $\widehat{b}=1,6099$. Desde que seu parámetro de forma é próximo de 1, sua taxa de falha ć aproximadamente constante, como pode ser visto na Figura 1.1. A tabela 1.4 fornece as estimativas da taxa de falha $\lambda(t)$ e a tabela 1.5 as estimativas da taxa de falha acumulada $A(t)$ calculadas via Kaplan-Meier, Nelson-Aalen e Weibull. 


\begin{tabular}{|c|c|c|c|}
\hline Tempos & Kaplan-Mcicr & Nelson-Aalen & Weibul1 \\
\hline \hline 19 & 0.033902 & 0.03333 & 0.000497 \\
\hline 43 & 0.035091 & 0.03448 & 0.000818 \\
\hline 44 & 0.036368 & 0.03571 & 0.000830 \\
\hline 148 & 0.037740 & 0.03704 & 0.001739 \\
\hline 169 & 0.039221 & 0.03846 & 0.001886 \\
\hline 171 & 0.040822 & 0.04000 & 0.001899 \\
\hline 205 & 0.042560 & 0.04167 & 0.002121 \\
\hline 232 & 0.044452 & 0.04318 & 0.002288 \\
\hline 248 & 0.046520 & 0.04545 & 0.002383 \\
\hline 250 & 0.048790 & 0.04762 & 0.0023395 \\
\hline 263 & 0.051293 & 0.05000 & 0.002470 \\
\hline 271 & 0.054067 & 0.052633 & 0.002515 \\
\hline 282 & 0.057158 & 0.05556 & 0.002577 \\
\hline 290 & 0.060625 & 0.05882 & 0.002621 \\
\hline 347 & 0.064539 & 0.06250 & 0.002925 \\
\hline 349 & 0.0668993 & 0.066667 & 0.002935 \\
\hline 398 & 0.074108 & 0.07143 & 0.003180 \\
\hline 477 & 0.080043 & 0.076942 & 0.003551 \\
\hline 514 & 0.087011 & 0.08333 & 0.003717 \\
\hline 595 & 0.095310 & 0.09091 & 0.004064 \\
\hline 603 & 0.105361 & 0.10000 & 0.004097 \\
\hline 662 & 0.117783 & 0.11111 & 0.004337 \\
\hline 700 & 0.133531 & 0.12500 & 0.004487 \\
\hline 701 & 0.154151 & 0.14286 & 0.004491 \\
\hline 706 & 0.182322 & 0.16667 & 0.004511 \\
\hline 709 & 0.223144 & 0.20000 & 0.004522 \\
\hline 710 & 0.287682 & 0.25000 & 0.004526 \\
\hline 7633 & 0.405465 & 0.333333 & 0.004729 \\
\hline 777 & 0.693147 & 0.50000 & 0.004782 \\
\hline 869 & $*$ & 1.00000 & 0.005120 \\
\hline
\end{tabular}

Tabela 1.4: Estimativas da taxa de falha via Kaplan-Meier, Nelson-Aalen e Weibull 


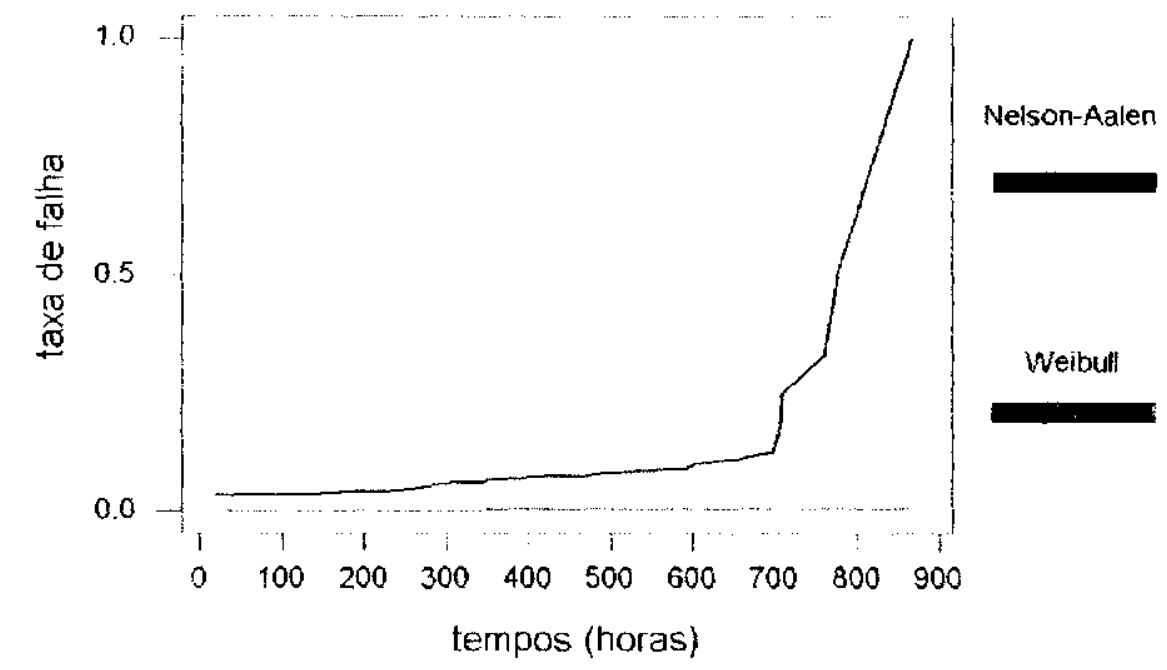

Figura 1.1: (irático das estimativas da taxa de falha via Velson- Aalen e Weibull

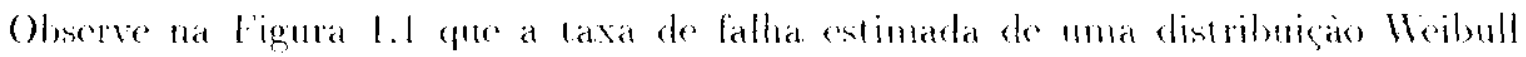

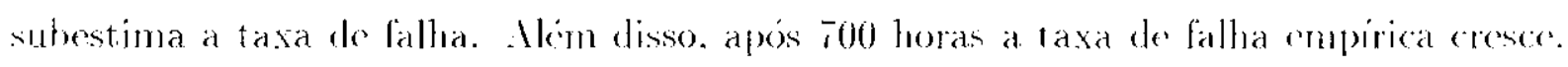
enquanto gue a taxa estimada via distribuiçào de Wribull permanece constante. 


\begin{tabular}{|c|c|c|c|}
\hline 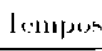 & 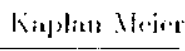 & 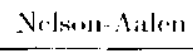 & Wrilull \\
\hline 19 & 0,0000 & 0.123 & $0.00=87$ \\
\hline $1 \% 3$ & $0.06 \times s+4$ & b) KKi & $10121 \times 6$ \\
\hline 4 & $(1.105 .34 ;$ & 0.19353 & $(0.0) 2269$ \\
\hline 1.15 & $0.14: 360$ & 0.11165 & 0.15992 \\
\hline 1699 & i.) $15: 232$ & (1). 129013 & (1). $1:+\times(10)$ \\
\hline $1 ; 1$ & 0.22314 & 0.219003 & 0.20170 \\
\hline 205 & $(1.2 \sin 70$ & (1.2) 2 (ntii) & $0.27(2)=0$ \\
\hline 232 & 0.31916 & 603417 & 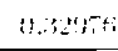 \\
\hline 213 & $0.33666^{-1}$ & 0.34963 & 1.:3671:? \\
\hline $2=11$ & 0.115 .47 & $1,29: 23$ & (1).371:191 \\
\hline $26: 3$ & $0.156 ; \% 6 ;$ & 0.4125 & (1). 4010554 \\
\hline 271 & $0.5168 \%$ & (1. 499888 & 0.4218 \\
\hline $2 *$ & 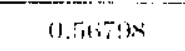 & $0.5 \times 4$ & 0.451 .19 \\
\hline 290 & 0.628661 & 0.611 .26 & $0.1-222$ \\
\hline 317 & $0.6 \% 3931=$ & $0.6 \overline{t i t a t i}$ & 1).63014 \\
\hline $3+9$ & (1) $-71: 11.4$ & $0.71 \times 12$ & 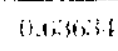 \\
\hline $39 \%$ & $0 . \times 362 \pi$ & $0.81 .1 \times ;$ & 0.78623 \\
\hline $\bar{H}$ & $(1.991629$ & $0.8 \div 17 \times$ & 1.0 .2231 \\
\hline 314 & 1.0093341 & (1) & $1.1 \times t: 79$ \\
\hline 595 & $1.09 \$ 61$ & 1.146602 & 1.56207 \\
\hline (5i):3 & 1. 2010597 & 1.1656112 & $1.53: 342$ \\
\hline (f) & 1.32176 & 1.27713 & $1.7 \times 35$ \\
\hline $7(m)$ & $1.45: 529$ & $1.1021 \%$ & $1,5,2 \pi$ \\
\hline 701 & 1 . firstit & 1.3.twy & 1.9.1. \\
\hline 70k: & 1.89186 & 1.7165 & $1.9782 x$ \\
\hline in: & $2.011: 41$ & 1.9116 & 1.991913 \\
\hline 710 & 2.61259 & $2.11 ; 1 ; i 5$ & $1,3: 36,5 ;$ \\
\hline $86: 3$ & $2.70 \times 15$ & $2.494 \times 3$ & 2.24167 \\
\hline 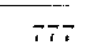 & $3+11124$ & $2.991: 1 * 0$ & $2.3101 \times 24 i$ \\
\hline 86,9 & * & 3409109 & 17634 \\
\hline
\end{tabular}

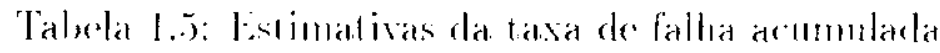

A. liguras 1.2 e 1.3 most ram. respectivanente as estimavas da funcio de confiabilidade

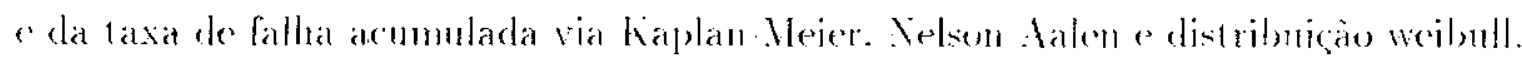




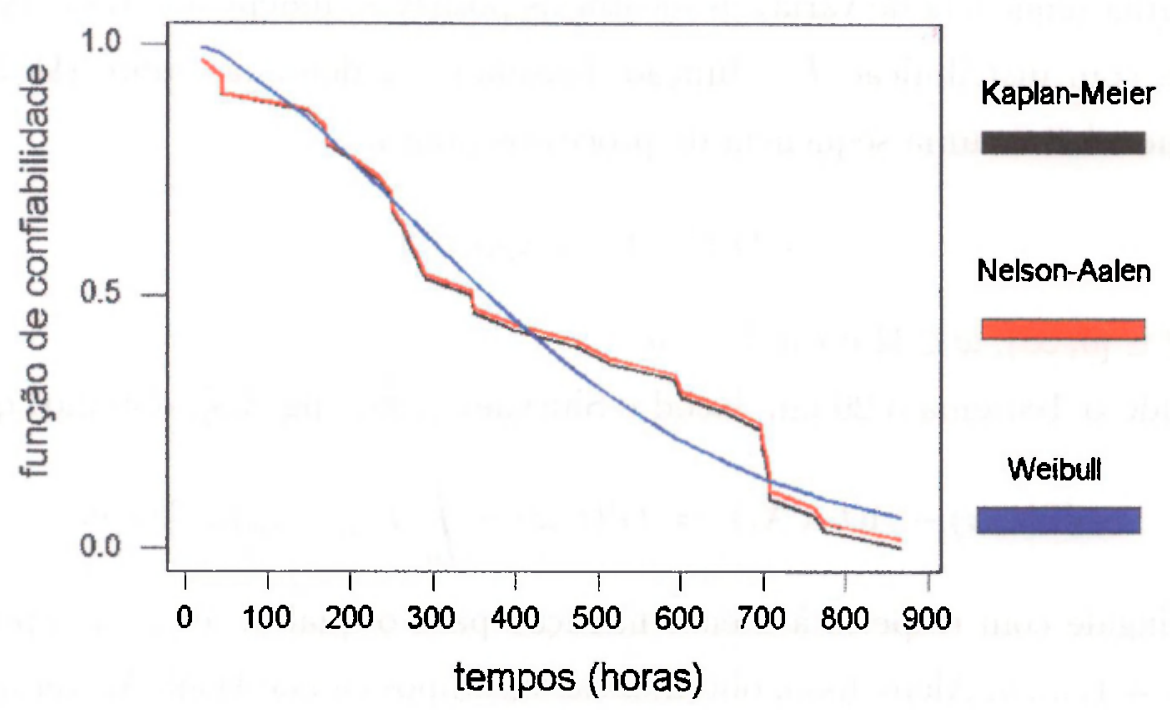

Figura 1.2: Gráfico das estimativas da função de confiabilidade

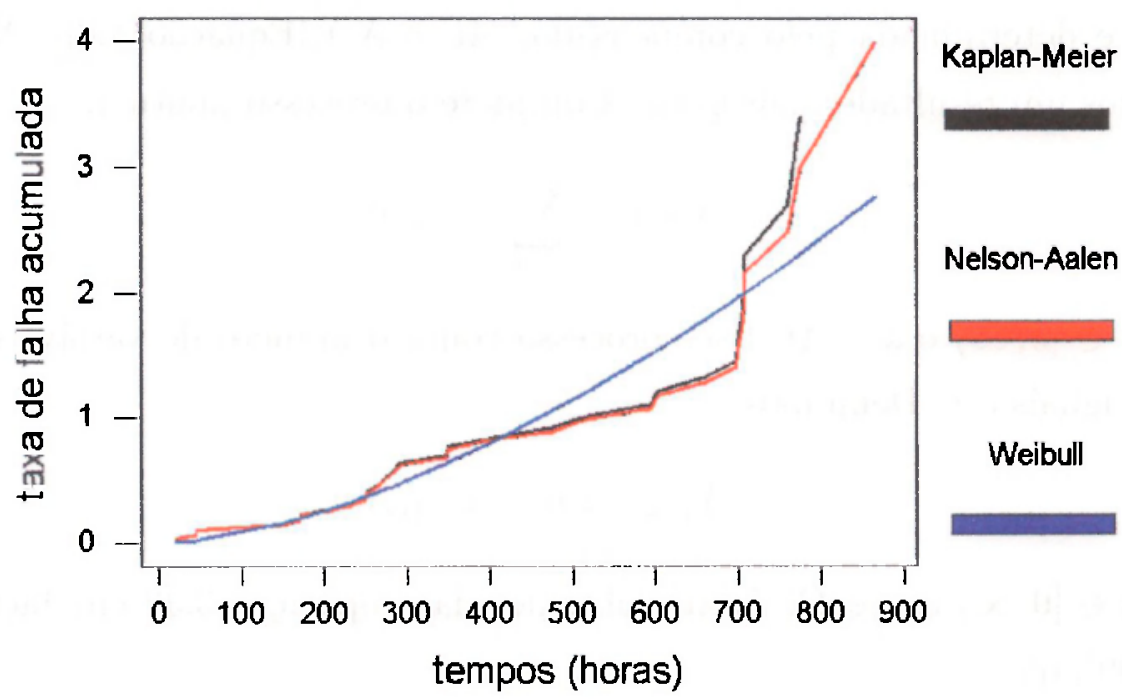

Figura 1.3: Gráfico das estimativas da taxa de falha acumulada

\subsection{A estimação não-paramétrica}

Neste trabalho, ao invés de utilizarmos os estimadores empíricos ou os estimadores paramétricos, vamos propor um estimador não paramétrico para a taxa de falha acumulada

$$
A(t)=\int_{0}^{t} \lambda(s) d s
$$




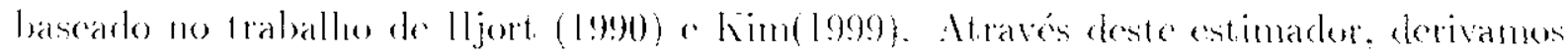

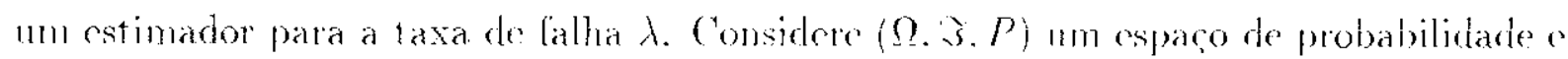

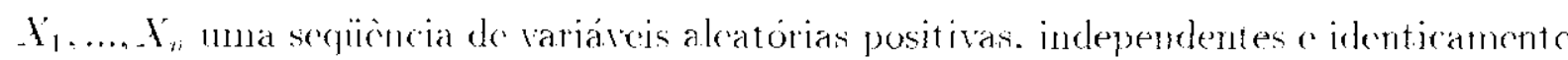

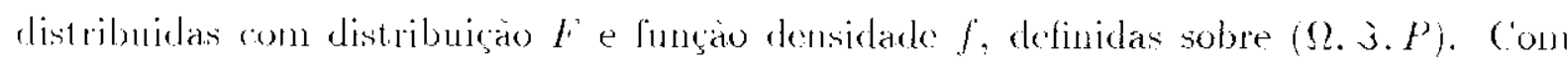
inso, podemos definir una secfüencia de processos pontuais

$$
\left(i(1 . \omega)=L_{\{x, y)}(\omega)\right.
$$

para todo $t \in[0 . \infty)$. w $\in \Omega(i-1 \ldots \ldots$.

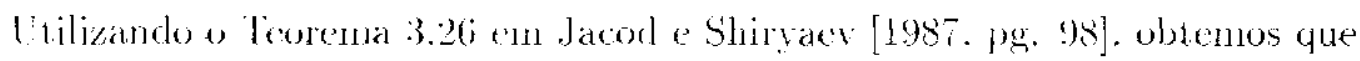

$$
\left.G^{i}(l, \omega)-A\left(1 \wedge X_{i}\right)=G(1, \omega)-\int_{1}^{1} I_{\{x}(\omega)>s\right\}(\omega) \lambda(*) d l \omega
$$

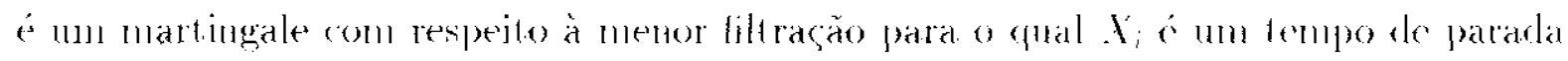
para todo $i=1, \ldots, n$. Além disso obtemos da decomposiçio de Doob-Mever que $.1\left(l \wedge X_{i}\right)$ Ćnnico. Como mostramos na seçào 1.1. a função de contiabilidade é dada por

$$
S(t)=\exp \{-A(t)\}=1-F(t) .
$$

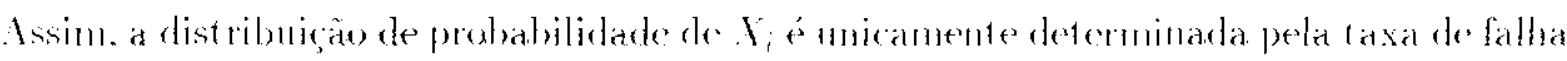
acumulada. Por outro lado. observamos que a distribuiçăo de probabilidade do processo

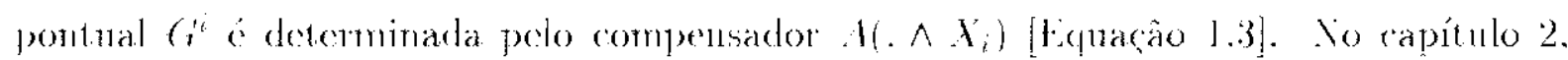
estabelecemos un resultado mais geral. Considere o processo pontual,

$$
N(\omega)-\sum_{i=1}^{\prime \prime}\left(i^{i}(t . \omega)\right.
$$

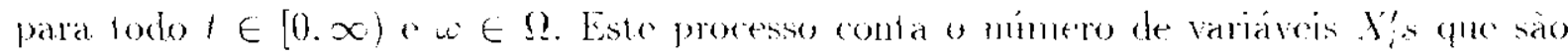
menores ou iguais a $l$. Definimes.

$$
Y_{s}(\omega)=n-Y_{s}(w)
$$

para todo $i \in[0, \infty]$ e $w \in \Omega$. Assim, obtemos da proposiça 3.32 m macod o Shiryate [1985. pg. 99]. (1)

$$
H_{i}=V_{i}-\int_{0}^{t} r_{s} \lambda(s) d s
$$

$\therefore$ um martingale com respeite à filtragào gerada por N. Além disso, sabemos que a distribuiço de probabilidade do processo. I' i determinada (on caracterizala) pelo com pensarlor [ver c apín ulo 2]

$$
\Lambda_{1}(\omega)=\int_{0}^{t} Y_{s} \lambda(s) d s=\int_{0}^{t} r_{s} d \cdot I_{s}
$$




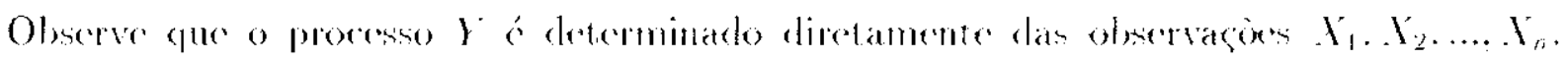
Ascim, para o compensador lo processo pontual basta doterminarmos a taxa de falha atcumuladal.

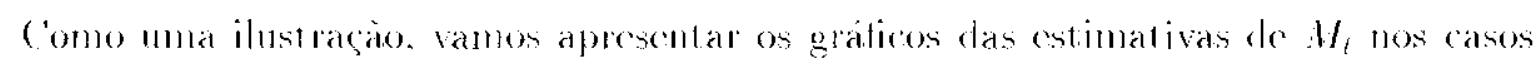

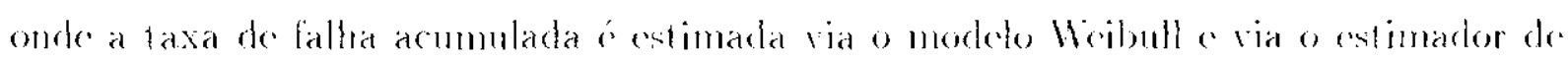

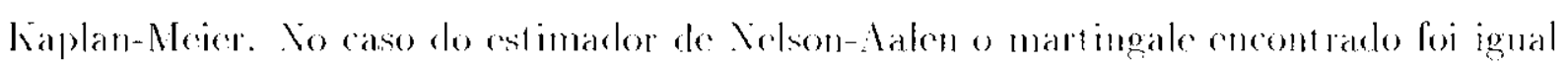
a zoro. As cstimativas de

$$
H_{t}=v_{i}-\int_{0}^{i} y_{s} d l_{s}
$$

foram oltidas conforme o procedinemto:

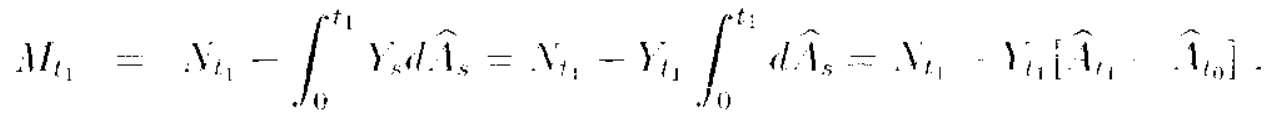

$$
\begin{aligned}
& M_{t_{2}}-N_{t_{2}}-\int_{11}^{t_{2}} Y_{s} d \hat{A}_{s}=N_{t_{2}}-\left[\int_{11}^{t_{1}} Y_{s} d \hat{A}_{s}+\int_{t_{1}}^{t_{2}} Y_{s} d \hat{l}_{s}\right]
\end{aligned}
$$

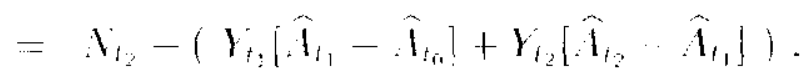

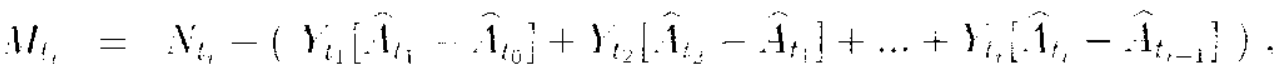

e assim por diante. 


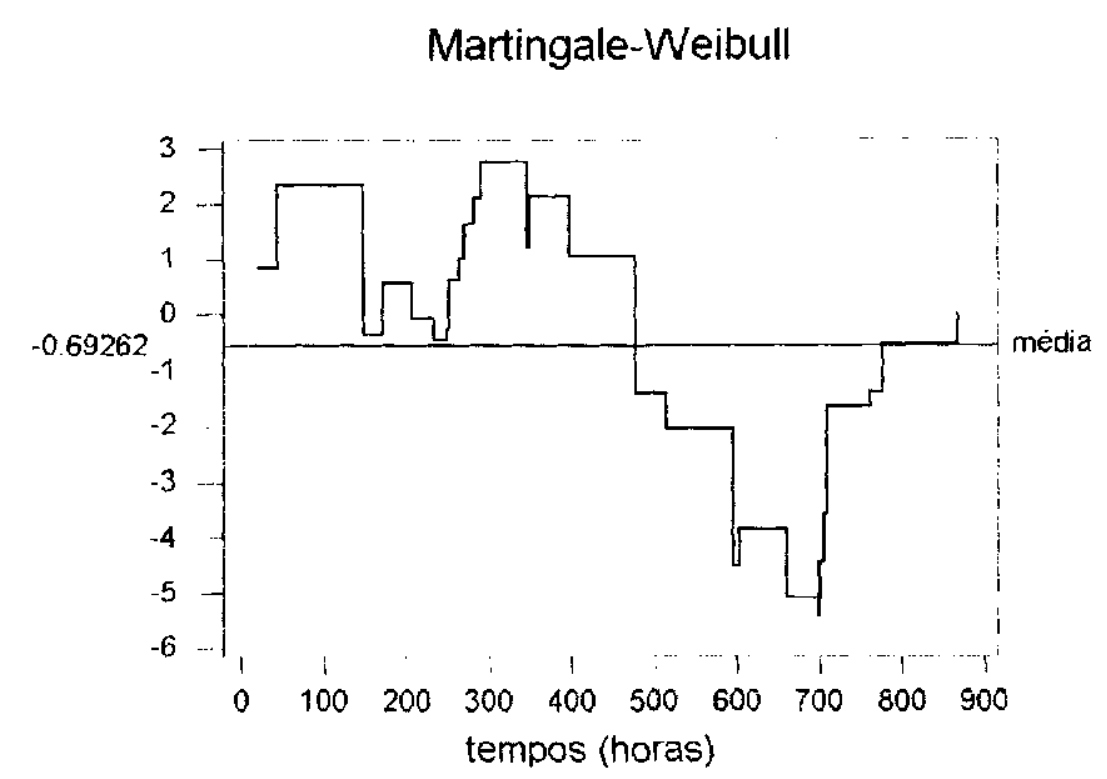

Figura 1.1: Mardingale pata a laxa actumbtada rle uma Wiribull

\section{Martingale-Kaplan-Meier}

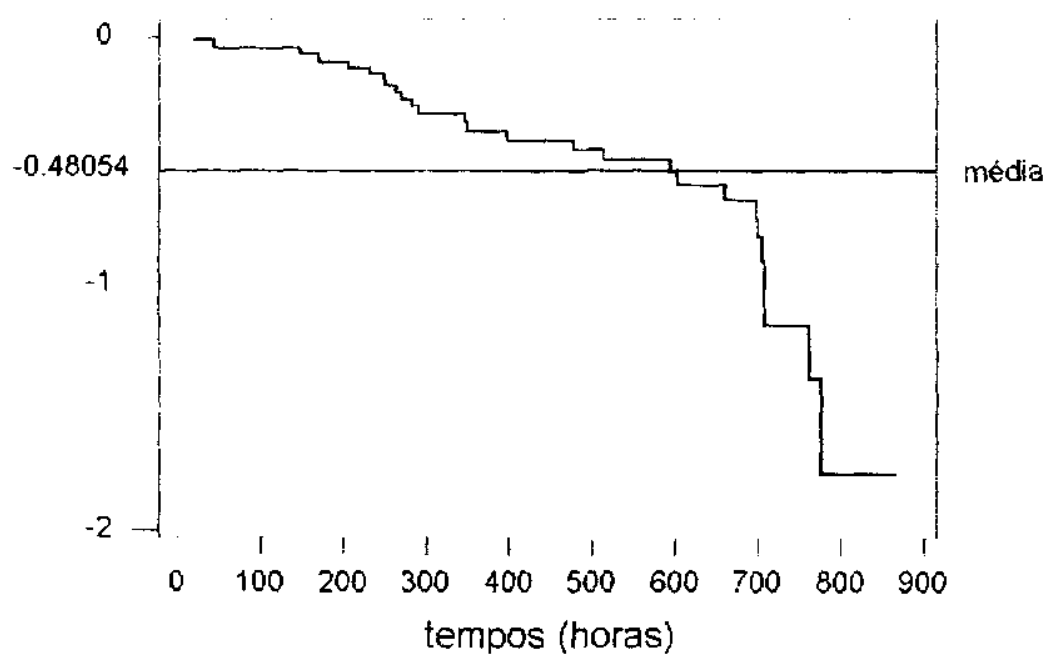

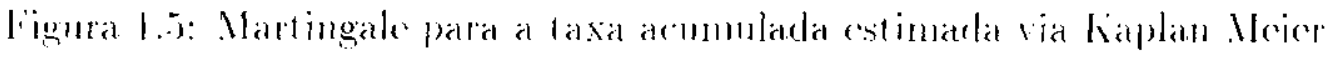

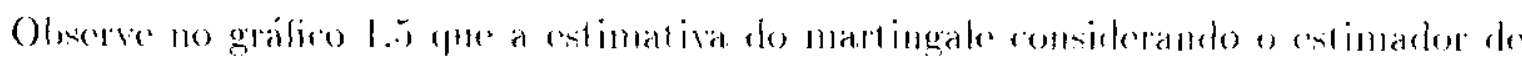
Kaplan Meier é negativo, o que sugere que o estimador de Kaplan-.Keier scja um esti-

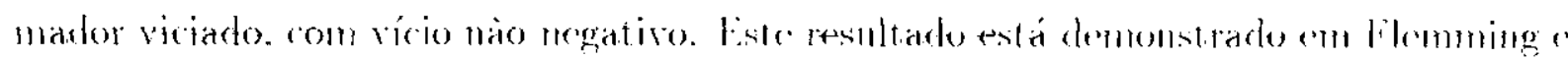


Harrington [Lema 3.2.1, pg. 99].

\subsubsection{Modelo Estatístico}

A seguir, vamos apresentar um modelo para descrever os dados. Considere $\left(x_{1}, x_{2}, \ldots, x_{n}\right)$ as observaçōes do tempo de falha. Conforme proposto anteriormente, vamos admitir que não temos falhas coincidentes, isto é, $x_{i} \neq x_{j}$ para todo $i \neq j=1, \ldots, n$. Sejam $t_{1}<t_{2}, \ldots<t_{n}$ a ordenação das observações $\left(x_{1}, x_{2}, \ldots, x_{n}\right)$. A ordenação é feita para simplificar a notação e esta não altcra o processo pontual associado,

$$
\sum_{i=1}^{n} I_{\left\{x_{i} \leq s\right\}}=\sum_{i=1}^{n} I_{\left\{t_{i} \leq s\right\}}
$$

para todo $s \in[0, \infty)$.

Considere $0=t_{0}<t_{1}<\ldots<t_{k} \in[0, \infty)$ os sucessivos tempos de falha de itens em teste, para algum $k \in \mathbb{N}$, onde $\mathbb{N}$ é o conjunto dos números naturais. Assim, definimos uma função $x:[0, \infty) \rightarrow S^{\infty}$ por

$$
\begin{gathered}
x(0)=(0,0,0, \ldots) \\
x(s)=\left(I_{\left.\left\{t_{1}, \infty\right)\right\}}(s), \ldots, I_{\left\{\left[t_{k}, \infty\right)\right\}}(s), 0,0, \ldots\right) ; s \geq 0
\end{gathered}
$$

onde $I_{\{\}}($.$) é a funçäo indicadora de conjunto e S^{\infty}=\{0,1\} \times\{0,1\} \times \ldots$ corresponde ao espaço de Cantor (ver apêndice).

Denotamos por $\Omega=\left\{x:(0, \infty) \longrightarrow S^{\infty} \mid 0=t_{0}<t_{1}<\ldots<t_{k} \in[0, \infty), k \in \mathbb{N}\right\}$, o espaço de trajetórias e sobre ele vamos definir uma $\sigma$-álgebra $\Im$. Tomamos uma classe $\Theta$ de subconjuntos de $\Omega$

$$
\Theta=\left\{C\left[k ; n ; w_{n}\right]: w_{n} \in S, k \in \mathbb{Q}, k \geq 0, n \in \mathbb{N}\right\},
$$

onde $C\left[k ; n ; w_{n}\right]-\left\{x \subset \Omega: x(k) \in \pi_{n}^{-1}\left(\left\{w_{n}\right\}\right)\right\}$ são chamados de cilindros e $\mathbb{Q}$ é o conjunto dos números racionais. Aplicando algumas operações de conjunto em $\Theta$ construímos uma álgebra $\Upsilon$, chamada álgebra dos cilindros e a $\sigma$-álgebra $\Im$ sobre $\Omega$ é a $\sigma$-álgebra gerada por $\Upsilon$, isto é $\Im-\sigma\{\Upsilon\}$. A filtração gerada pelas trajetórias do processo pontual é definida pela seguinte família crescente de $\sigma$-álgebras

$$
\Im_{t}=\sigma\left\{C\left[k ; n ; w_{n}{ }^{\jmath}, w_{n} \in S, k \in \mathbb{Q} \cap[0, t], k \geq 0, n \in \mathbb{N}\right\}, t \in[0, \infty) .\right.
$$

No capítulo 2 faremos uma análise detalhada do espaço de trajetórias e suas propriedades. Assim, para definirmos o processo pontual considere uma probabilidade $P$ sobre $(\Omega, \Im)$ e 
$T_{j}: \Omega \longrightarrow[0, \infty)$ uma variável aleatória definida por

$$
\begin{aligned}
T_{j}(x) & =\inf \{s \in[0, \infty]: x(s)=\underbrace{(1,1,1, \ldots, 1}_{j \text { vezes }}, 0,0, \ldots)\}= \\
& =t_{j} ; \text { se } j \leq k \\
& =+\infty ; \text { se } j>k .
\end{aligned}
$$

Esta variável aleatória é um tempo de parada [Teorema 2.2.1] e o processo definido por

$$
N_{t}(x)=\sum_{j=1}^{\infty} I_{\left\{T_{j}<t\right\}}(x), x \in \Omega, \quad t \in[0, \infty]
$$

em $\left(\Omega, \Im_{t}, \Im, P\right)$ é um processo pontual

O processo $N_{t}$ é um submartingale e de acordo com a decomposição de Doob-Meyer, Liptser e Shiryayev [1978, Teorema 3.8, pgr. 65], existe $11 \mathrm{~m}$ único processo previsível crescente $\Lambda_{i}$, tal que

$$
M_{t}=N_{t}-\Lambda_{t}
$$

é um martingale quadrado integrável. Aalen (1978) considera que o processos taxa de falha possa ser escrito como o produto de uma função determinística não-negativa desconhecida $\lambda$ e um processo estocástico observável $Y$ sobre um intervalo de tempo. Este modelo é denominado modelo de intensidade multiplicativa. Assim, temos que

$$
\Lambda_{t}(x)=\int_{0}^{t} \lambda(s) Y_{s}(x) d s-\int_{0}^{t} Y_{s}(x) d A_{s}, t \in[0, \infty), x \in \Omega .
$$

Observe que o modelo de intensidade multiplicativa generaliza o modelo apresentado na Equação 1.4. Aalen propôs que ao invés de $\lambda$ fosse estimada uma função dela

$$
A_{t}=\int_{0}^{t} \lambda(s) d s .
$$

Utilizando a decomposição de Doob-Mcyer podemos escrever de modo heurístico que $d N_{t}=d M_{t}+d A_{t}=d M_{t}+\lambda(t) Y_{t} d t . \Lambda$ través do método dos momentos, obtemos como estimador para $A_{t}$,

$$
\widehat{A}_{t}=\int_{0}^{t} \frac{d N_{s}}{Y_{s}}
$$

Levando em conta que $\mathrm{Y}$ possa assumir um valor nulo, resscrevermos o estimador $\hat{A}_{t}$ na forma.

$$
\widehat{A}_{\ell}=\int_{0}^{t} \frac{J_{s} d N_{s}}{Y_{s}}
$$


onde $J_{s}=\lim _{h \downarrow 0} I_{\left\{Y_{s-h}>0\right\}}$ e quando $Y_{s}=0$ definimos $\frac{I_{s}}{Y_{s}}=0$. Esse estimador corresponde ao estimador 1.2, que é estimador de Nelson-Aalen na forma de uma integral de Stieltjes. Utilizando a decomposição de Doob-Meyer, obtemos que,

$$
\int_{0}^{\iota} \frac{J_{s} d M_{s}}{Y_{s}^{-}}=\int_{0}^{t} \frac{J_{s} d N_{s}}{Y_{s}}-\int_{0}^{t} \frac{J_{s} d \Lambda_{s}}{Y_{s}}
$$

é um martingale quadrado integrável. Assim,

$$
E\left[\int_{0}^{t} \frac{J_{s} d N_{s}}{Y_{s}}\right]=E\left[\int_{0}^{t} \frac{J_{s} d \Lambda_{s}}{Y_{s}}\right]
$$

Agora, vamos calcular o erro quadrático médio e apresentar um estimador para a variância do estimador de Nelson-Aalen.

$$
E\left[\left(\int_{0}^{t} \frac{J_{s} d N_{s}}{Y_{s}}-\int_{0}^{t} \frac{J_{s} d \Lambda_{s}}{Y_{s}}\right)^{2}\right]=E\left[\left(\int_{0}^{t} \frac{J_{s} d M_{s}}{Y_{s}}\right)^{2}\right] .
$$

Desde que

$$
\int_{0}^{t} \frac{J_{s} d M_{s}}{Y_{s}}
$$

é um martingale quadrado integrável, concluímos da decomposição de Doob-Meyer, do Teorena $2.4 .2 \mathrm{~cm}$ Flemming e Harrington (1991, pg.67) e do modelo de intensidade multiplicativa, que

$$
\begin{aligned}
E\left[\left(\int_{0}^{t} \frac{J_{s} d M_{s}}{Y_{s}}\right)^{2}\right] & =E\left[\int_{0}^{t} \frac{J_{s}^{2} d \Lambda_{s}}{Y_{s}^{2}}\right]=E\left[\int_{0}^{t} \frac{J_{s} Y_{s} d A_{s}}{Y_{s}^{2}}\right] \\
& =E\left[\int_{0}^{t} \frac{J_{s} d A_{s}}{Y_{s}}\right] .
\end{aligned}
$$

Substituindo $d A_{s}$ por $J_{s} d N_{s} / Y_{s}$, obtemos um estimador empírico para a variância do estimador de Nelson-Aalen, conforme descrito abaixo

$$
\widehat{\operatorname{Var}}[\widehat{\Lambda}(t)]=\int_{0}^{t} \frac{J_{s} d N_{s}}{Y_{s}^{2}} .
$$

Maiores informaçòes sobre o estimador de Nelson-Aalen, como a consistência assintótica, podem ser encontradas em Aalen (1978) e Flemming e Harrington (1991).

\subsubsection{Estimador de Bayes}

Ao invés de utilizarmos o estimador não paramétrico de Nelson-Aalen, vamos utilizar um estimador Bayesiano não-paramétrico para a taxa de falha acumulada $A$, conforme proposto em Hjort (1990) e Kim (1999). Além disso, vamos derivar um estimador para a taxa de falha $\lambda$. 
Sejam $X_{1}, \ldots, X_{n}$ variáveis aleatórias positivas independentes e identicamente distribuídas com função de distribuição $F$. O objetivo consiste em estimar $F$, onde o parâmetro de interesse toma valores em um espaço de funçōes. Assim, para aplicar o enfoque Bayesiano, precisamos definir uma probabilidade sobre o espaço das funções de distribuição acumulada. Para isto, basta construirmos um processo estocástico cujas trajetórias são funções de distribuição acumulada. Neste sentido, destacamos os processos de Dirichlet [Ferguson (1973),(1974)], processos tailfree e neutral to the right, [Doksum (1974)]. Se $F$ é absolutamente contínua com função densidade $f$, Dikstra e Laud (1981) utilizaram processos gama estendidos como distribuição à priori para a taxa de falha $\lambda$. Ao invés do estimar a função de distribuição acumulada $F$, Hjort (1990) propòs um método Bayesiano para estimar a taxa de falha acumulada $A$. Se a $F$ é absolutamente contínua, entäo, os resultados obtidos para estimar $F$ podem ser facilmente adaptados para estimar $A$, pois

$$
F(t)=1-\exp \{-A(t)\}, \forall t \in[0, \infty) .
$$

Além disso, sabemos que $F$ é absolutamente contínua se, e somente se, $A$ também é absolutamente contínua. Por outro lado, todos os processos estocásticos utilizados para definir a distribuição à priori são processos de Lévy puro salto. Desta forma, os resultados apresentados para estimar $F$ não são diretamente adaptados para estimar $A$ e vice-versa. Assim, todos os processos estocásticos à priori apresentam trajetórias do tipo escada, consequentemente não são absolutamente contínuas. Neste trabalho, vamos apresentar métodos para estimar a taxa de falha acumulada $A$, como aprescntado em IIjort (1990) Kim (1999).

Considere o espaço de probabilidade $(\Omega, \Im, P)$ definido na sç̧āo 2.2 , vamos assumir que o processo pontual $N_{t}$ está definido em $[0,1]$; embora ele possa ser generalizado para o intervalo $[0, \tau]$ e que o processo $\Lambda_{t}$ satisfaz o modelo de intersidade multiplicativo

$$
\Lambda_{t}=\int_{0}^{t} Y_{s} d A(s)
$$

onde $Y$ é um processo previsível (observável) e $A$ é uma função determinística desconhecida, denominada taxa de falha acumulada. Abaixo, definimos o espaço das taxas de falha acumulada.

$$
\begin{aligned}
\mathcal{A}= & \{\text { classe das funçôes não decrescentes } A \text { contínuas à direita } \\
& \text { definidas em }[0,1], \Delta A \leq 1, A(0)=0\}
\end{aligned}
$$

Para esta classe considere $\Sigma_{\mathcal{A}}$ a $\sigma$-álgebra gerada pelos cilindros. O espaço das observações corresponde ao $\Omega$ definido na seção $2.2 \mathrm{com}$ a $\sigma$-álgehra associada $\Im$. Para definir 
uma probabilidade à priori sobre $\Sigma_{\mathcal{A}}$, vamos construir um processo estocástico com trajetórias em $\mathcal{A}$ chamados de processo de Lévy puro salto, conforme a definição abaixo

Definição 1.2.1 (Processo de Lévy puro salto). Um Processo de Lévy puro salto é um processo estocástico $A$, satisfazendo:

a) A é càdlàg (contínuo à direita e com limites à esquerda) e adaptado à filtração $\Im_{t:} t \geq 0, A \in \mathcal{A}$;

b) Para todo $t \in[0,1]$ ew $\in \Omega$

$$
A_{l}(\omega)=\sum_{s} \Delta A_{s}(\omega) I_{\left\{\Delta A_{s}(\omega)>0\right\}}(\omega) I_{\{[0, c]\}}(s)
$$

c) A tem incrementos independentes;

d) $A_{0}(\omega)=0 ; \Delta A_{t}(\omega)<1 ; \forall \omega \in \Omega ; \forall t \in[0,1]$.

No Capítulo 3, fazomos um estudo detalhado sobre os processos de Lévy puro salto e suas representaçôes. Esta classe de processos foi introduzida por Lévy e pode ser caracterizada pela estrutura da função característica. Também neste capítulo, mostramos que a classe de processos de Lévy puro salto pode ser caracterizada pelo compensador de saltos $\nu$, também conhecido como medida de Lévy. Para isso, associamos uma medida aleatória $\mu$, na forma

$$
\mu(\omega ;[0, t] \times B)=\sum_{s} I_{\left\{\Delta A_{s}(\omega)>0\right\}}(\omega) I_{\{0, t\}\}}(s) I_{\{B\}}\left(\Delta A_{s}(\omega)\right),
$$

para $\omega \in \Omega, t \in[0,1], B \in \beta((0,1])$ e o compensador

$$
\nu([0, t] \times B)=E[\mu(. ;[0, t] \times B)], B \in \beta((0,1]) .
$$

da medida alcatória $\mu$. Do lema 3.1.3 temos que

$$
\int_{0}^{1} x \mu\left(\omega:[0, t j \times d x)=A_{t}(\omega), t \in[0,1], \omega \in \Omega .\right.
$$

Dessa forma o processo de Lévy $A$ é totalmente caracterizado pelo compensador $\nu$. O processo beta é um processo de Lévy com o compensador definido por

$$
\nu([0, t] \times B)=\int_{0}^{t} \int_{D} \frac{1}{x} b(x: \alpha(s), \beta(s)) d x d A_{0}(s), \quad t \in[0,1], \quad B \in \beta((0,1])
$$

onde

$$
b(x: a, b)=\frac{\Gamma(a+b)}{\Gamma(a) \Gamma(b)} x^{u-1}(1-x)^{b-1}, 0<x<1 .
$$


ć a funçāo densidade da distribuição beta com parâmetros $a>0$ e $b>0$,

$$
A_{0}=\int_{0}^{t} \lambda_{0}(s) d s
$$

é uma taxa de falha acumulada inicial e $\alpha, \beta$ são funçoes contínuas estritamente positivas definidas $\mathrm{cm}[0,1]$. Alćm disso, a classe dos processos de Lévy é conjugada [Doksum (1974), Ferguson e Phadia (1979)]. Além disso, o processo beta também é conjugado e o compensador à posteriori ó dado por [Kim (1999)]:

$$
\begin{aligned}
\nu^{p}([0, l] \times D)= & \int_{0}^{t} \int_{D}(1-x)^{Y_{s}} \frac{1}{x} b(x: \alpha(s), \beta(s)) d x d A_{0}(s) \\
& +\int_{0}^{t} \int_{D} \frac{1}{x} b\left(x: \alpha(s), \beta(s)+Y_{s}-1\right) d x d N_{s},
\end{aligned}
$$

$N_{s}=\sum_{i} I_{\left\{T_{i} \leq s\right\}}$ \& $Y_{s}=\sum_{i} I_{\left\{T_{i} \geq s\right\}}=n-N_{s-}$.

Assim, o estimador de Bayes para o processo beta com relação à função de perda quadrática será dado pela esperança da distribuição à posteriori. Logo, do Teorema 3.2.1, temos que o estimador de Bayes é

$$
\begin{aligned}
\widehat{A}_{t}= & E\left[A_{t} \mid N\right]=\int_{0}^{t} \int_{0}^{1} x \nu^{P}(d s, d x)=\int_{0}^{t} \int_{0}^{1}(1 \cdots x)^{Y_{s}} b(x: \alpha(s), \beta(s)) d x d A_{0}(s) \\
& +\int_{o}^{t} \int_{0}^{1} b\left(x: \alpha(s), \beta(s)+Y_{s}-1\right) d x d N_{s} \\
= & \int_{0}^{t} \frac{\Gamma(\alpha(s)+\beta(s)) \Gamma\left(\beta(s)+Y_{s}\right)}{\Gamma(\beta(s)) \Gamma\left(\alpha(s)+\beta(s)+Y_{s}\right)} \lambda_{0}(s) d s+\int_{0}^{t} \frac{\alpha(s)}{\alpha(s)+\beta(s)+Y_{s}-1} d N_{s} .
\end{aligned}
$$

Kim e Lee (2002) mostraram que $\widehat{A}_{t}$ é consistente para estimar $A_{t}$ se, e somente se, $\alpha(s)=1, \forall s \in[0,1]$. Além disso, quando $\alpha(s)-1$ obtemos o processo beta de: Hjort(1990) com estimador de Bayes dado por:

$$
\widehat{A}_{t}=\int_{0}^{t} \frac{\beta(s) \lambda_{0}(s)}{\beta(s)+Y_{s}} d s+\int_{0}^{t} \frac{d N_{s}}{\beta(s)+Y_{s}^{r}} .
$$

Vamos assumir que as taxa de falha acumulada e taxa de faltha iniciais $A_{0}(s)$ e $\lambda_{0}(s)$ são da distribuição Weibull, e dadas respectivamente por

$$
A_{0}(s)=\left(\frac{s}{a}\right)^{b}
$$

$$
\lambda_{0}(s)=\frac{b}{a^{b}} s^{b-1} .
$$

Além disso, propomos que a função $\beta(s)$ seja determinada por $n S(s)$ onde

$$
S(s)=\exp \left\{-\left(\frac{s}{a}\right)^{b}\right\} \text {, }
$$


é a função de confiabilidade da distribuição Weibull, pois, conforme notado por Hjort [1990, pg. 1264 ], $\beta(s)$ pode ser interpretada como o conjunto de risco em $s$ à priori. Vamos considerar $v=1 / a^{b}$ para simplificar a notação. Logo,

$$
\widehat{A}_{t}=\int_{J_{0}^{t}}^{t} \frac{n \exp \left\{-\widehat{v} s^{b}\right\} \hat{v} \hat{b}^{b-1}}{n \exp \left\{-\widehat{v} s^{\hat{b}}\right\}+Y_{s}} d s+\int_{0}^{t} \frac{d N_{s}}{n \exp \left\{-\widehat{v} s^{\hat{b}}\right\}+Y_{s}}
$$

onde $\widehat{a}$ e $\widehat{b}$ são os estimadores de máxima verossimilhança e $\widehat{v}=1 / \widehat{a}^{\hat{b}}$.

Além disso no capítulo 4 propomos um estimador para a taxa de falha como sendo

$$
\begin{aligned}
\widehat{\lambda}_{s} & =\frac{\beta(s) \lambda_{0}(s)}{\beta(s)+Y_{s}} ! \frac{1}{\beta(s)+Y_{s}} \\
& =\frac{n \exp \left\{-\widehat{v} s^{b}\right\} v \widehat{b s}^{b-1}}{n \exp \left\{-\widehat{v} s^{b}\right\}+Y_{s}}+\frac{1}{n \exp \left\{-\widehat{b}^{b}\right\}+Y_{s}} .
\end{aligned}
$$

As estimativas de máxima verossimilhança para os parâmetros $a$ e $b$ fapresentados na seção 1.1.3] - são, respectivamente, $\widehat{a}=462.13 \mathrm{e} \widehat{b}=1.6099, \log 0 \widehat{v} \simeq 5.128 \times 10^{-5}$.

O gráfico da Figura 1.6 mostra o comportamento dos estimadores da taxa de faIha acumulada estimada via Kaplan-Meier, via Weibull e via inferência Bayesiana nãoparamétrica [conforme 1.10] para os dados do freio do ERJ-145.

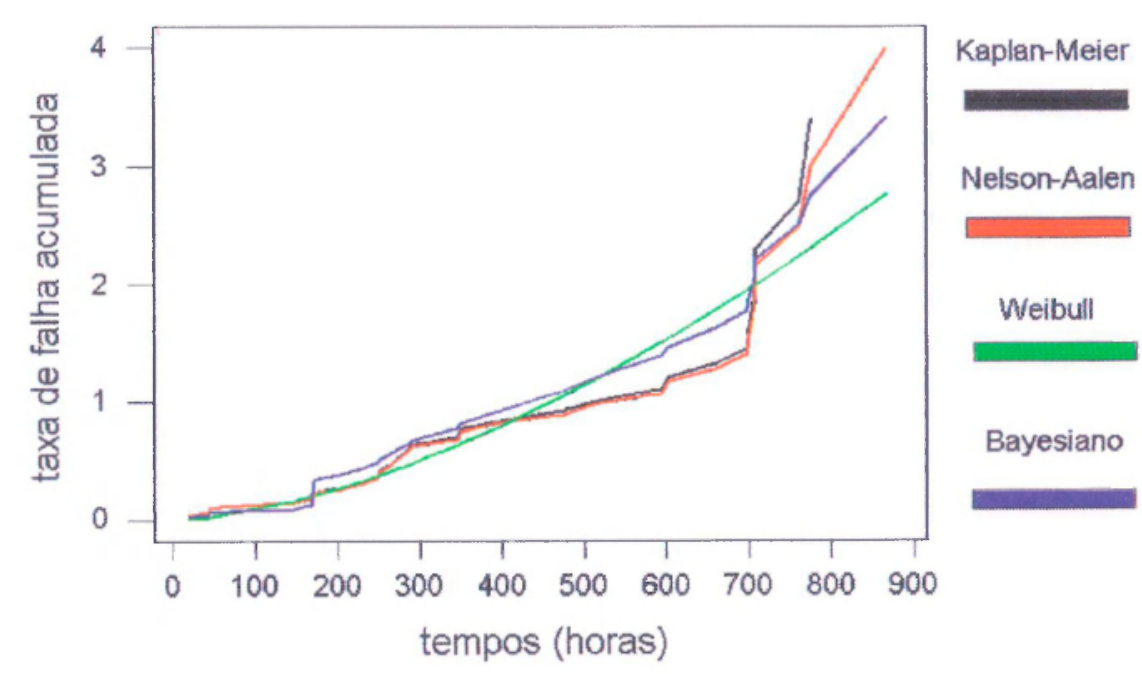

Figura 1.6: Gráficos da Taxa de Falha acumulada estimada via Kaplan-Meier, NelsonAalen, Weibull e Bayesiano Empírico 


\section{Capítulo 2}

\section{O Processo Pontual}

Neste capítulo iremos estudar o processo pontal, a decomposição de Doob-Meyer, a taxa de falha do processo pontual e apresentar os teoremas de existência e unicidade para o compensador do processo pontual. Para isso, deveremos ter bem definidos, o espaço amostral, a $\sigma$-álgebra e uma probabilidade com os quais iremos trabalhar.

\subsection{O Espaço de Trajetórias}

Considere $0=t_{0}<t_{1}<\ldots<t_{k} \in[0, \infty)$ para algum $k \in \mathbb{N}$, os sucessivos tempos de fallas de itens em teste. Assim, definimos a função $x:[0, \infty) \longrightarrow S^{\infty}$ por

$$
\begin{gathered}
x(0)=(0,0,0,0,0, \ldots) \\
x(s)=\left(I_{\left.\left\{i t_{1}, \infty\right)\right\}}(s), I_{\left.\left\{t_{2}, \infty\right)\right\}}(s), \ldots, I_{\left\{\left[t_{k}, \infty\right)\right\}}(s), 0,0,0, \ldots\right),
\end{gathered}
$$

onde $I_{1,\}}($.$) é a função indicadora de conjunto.$

A função $x$ definida acima representa todas as possibilidades de ocorrência para um experimento de confiabilidade onde, a cada item do experimento corresponde um tempo de falha $t_{3}$

Lema 2.1.1. A funçäo $x:[0, \infty) \longrightarrow S^{\infty}$ é mensurável.

Dem:

$$
\begin{aligned}
x^{-1}\left[\pi_{j}^{-1}(\{1\})\right]=\left[t_{j}, \infty\right) ; & j \leq k \\
x^{-1}\left[\pi_{j}{ }^{1}(\{0\})\right]=\left[0, t_{j}\right) ; & j<k \\
x^{1}\left[\pi_{j}^{-1}(\{1\})\right]=\varnothing ; & j \geq k+1 \\
x^{-1}\left[\pi_{j}^{-1}(\{0\})\right]=[0, \infty) ; & j>k+1
\end{aligned}
$$


Mostramos que para qualquer $C \in \mathcal{H}$ (ver Apêndice) temos, $x^{-1}(C) \in \beta([0, \infty))$. Temos que mostrar que qualquer que seja $A \in \mathcal{G}$ (ver Apendice) temos $x^{-1}(A) \in \beta([0, \infty)$ ). Considere então,

$$
\left.\mathcal{E}=\left\{A \subset S^{\infty}: x^{-1}(A) \in \beta(0, \infty)\right)\right\} .
$$

Dessa definição temos que $\mathcal{H} \subset \mathcal{E}$, então

$$
\mathcal{G}=\sigma(\mathcal{H}) \subset \sigma(\mathcal{E})
$$

Se $\mathcal{E}$ for uma $\sigma$-álgebra então $\mathcal{E}=\sigma(\mathcal{E})$. Basta então mostrar que $\mathcal{E}$ é umá $\sigma$-álgebra. () conjunto vazio e $S^{\infty} \in \mathcal{E}$. Seja $A \in \mathcal{E}$ então, $x^{-1}\left(A^{c}\right)=\left[x^{-1}(A)\right]^{c}$, desde que $x^{-1}(A) \in$ $\beta\left([0, \infty)\right.$ ), temos que $A^{c} \in \mathcal{E}$. Considere agora $A_{1}, A_{2}, \ldots \in \mathcal{E}$ cntão,

$$
x^{-1}\left(\bigcup_{i=1}^{\infty} A_{i}\right)-\bigcup_{i=1}^{\infty}\left[x^{-1}\left(A_{i}\right)\right],
$$

desde que $x^{-1}\left(A_{i}\right) \in \beta([0, \infty))$, temos que $\bigcup_{i-1}^{\infty} A_{i} \in \mathcal{E}$. Portanto $\mathcal{E}$ i uma $\sigma$-álgebra .

Com isso fica definido nosso espaço de trajetórias, cujos elementos são as funções $x$, isto é

$$
\Omega=\left\{x:[0, \infty) \longrightarrow S^{\infty} \mid 0-t_{0}<t_{1}<\ldots<t_{k} \in[0, \infty), k \in \mathbb{N}\right\}
$$

Vamos construir agora uma estrutura de mensurabilidade sobre o espaço de trajetórias $\Omega$. Considere o cilindro

$$
C\left[k ; n ; w_{n}\right]=\left\{x \in \Omega: x(k) \in \pi_{n}^{-1}\left(\left\{w_{n}\right\}\right)\right\}
$$

onde $w_{n} \in S=\{0,1\}, k \in \mathbb{Q}, k \geq 0, n \in \mathbb{N}$.

Denotamos

$$
\Theta=\left\{C\left[k ; n ; w_{n}\right]: w_{n} \in S, k \in \mathbb{Q}, k \geq 0, n \in \mathbb{N}\right\}
$$

Definição 2.1.1. Uma classe $\mathcal{A}$ separa pontos em um conjunto $\Omega$ se, para todo $x, y \in \Omega$ distintos, existe $A \in \mathcal{A}$ tal que

$$
I_{\{A\}}(x)+I_{\{A\}}(y),
$$

onde $I_{\{.\}}($.$) é a função indicadora de conjunto.$

Lema 2.1.2. A classe $\Theta$ é enumerável e separa pontos no espaço $\Omega$.

Dem: Desde que $S$ é finito, $\mathbb{Q}$ cnumerável e $\mathbb{N}$ enumerável, obtemos do Teorema $2 \mathrm{~cm}$ Kolmogorov e Fomim [1975,pag. 12] que $\Theta$ também é enumerável. Na sequência, vamos 
mostrar que $\Theta$ separa pontos no espaço $\Omega$. Considere $x_{1}$ e $x_{2}$ elementos de $\Omega$ distintos, isto é, $x_{1}(s) \neq x_{2}(s)$, para algum $s \in[0, \infty)$. Assim, existem sequências

$$
0=t_{0}^{i}<t_{1}^{i}<\ldots<t_{k_{i}}^{i} \in[0, \infty)
$$

para algum $k_{i} \in \mathbb{N}, i=1,2$, tais que

$$
\begin{aligned}
& x_{1}(s)=\left(I_{\left.\left\{i t_{1}^{1}, \infty\right)\right\}}(s), I_{\left.\left\{i t_{2}^{2}, \infty\right)\right\}}(s), \ldots, I_{\left.\left\{t_{k_{1}}^{2}, \infty\right)\right\}}(s), 0,0,0, \ldots\right), s \in[0, \infty) \\
& x_{2}(s)=\left(I_{\left\{\left[t_{1}^{2}, \infty\right)\right\}}(s), I_{\left.\left\{\mid t_{2}^{2}, \infty\right)\right\}}(s), \ldots, I_{\left\{\left[t_{k_{2}}^{2}, \infty\right)\right\}}(s), 0,0,0, \ldots\right), s \in[0, \infty)
\end{aligned}
$$

Desde que $x_{1} \neq x_{2}$, o conjunto de pontos $\left\{t_{1}^{1}, \ldots, l_{k_{1}}^{1}\right\}$ é diferente do conjunto de pontos $\left\{t_{1}^{2}, \ldots, t_{k_{2}}^{2}\right\}$ para pelo menos um $t_{j}^{i}$. Considere $l o$ primeiro índice tal que $t_{l}^{1} \neq t_{l}^{2}$, admitimos que $t_{l}^{1}<t_{l}^{2}$. Desde que $\mathbb{Q}$ é denso em $[0, \infty)$, existe um número racional $m$ tal que $t_{l}^{1}<m<t_{l}^{2}$. Com isso, obtemos que

$$
I_{\{C[m ; i ; ;]\}}\left(x_{1}\right) \neq I_{\{C\{[m ; ; ; 1]\}}\left(x_{2}\right),
$$

onde $I_{\{.\}}($.$) é a função indicadora de conjunto. Portanto, a classe \Theta$ separa pontos em $\Omega$.

Na seqüiência, vamos definir una classe de subconjuntos de $\Omega$ satisfazendo a propriedade de compacidade introduzida por Marczewski(1953). Esta propriedade será utilizada para a construção de probabilidades sobre o espaço de trajetórias.

Definição 2.1.2. Uma classe $\mathcal{A}$ de subconjuntos de um conjunto $\Omega$ é dita ser compacta se para cada sequência $\left\{C_{n}, n \geq 1\right\}$ em $\mathcal{A}$ com interseç̧āo $\bigcap_{n=1}^{\infty} C_{n}=\varnothing$ existe um inteiro $N$ tal que $\bigcap_{n=1}^{N} C_{n}=\varnothing$

$\Lambda$ seguir, vamos mostrar que a classe $\Theta$ satisfaz a propriedade da compacidade.

Lema 2.1.3. A classe $\Theta$ é compacta.

Dem: Considere a sequência $\left\{C_{n}\right\} \subset \Theta$ com $\bigcap_{n=1}^{\infty} C_{n}=\varnothing$. Então, existem dois índices $j_{1}$ e $j_{2} \in \mathbb{N}$ tais que

$$
\begin{aligned}
& C_{j_{1}}=\left\{x \in \Omega: x(k) \in \pi_{n}^{-1}(\{1\})\right\} \\
& C_{j_{2}}=\left\{x \in \Omega: x(k) \in \pi_{n}^{-1}(\{1\})\right\}
\end{aligned}
$$


para algum $k \in \mathbb{Q} \subset n \in \mathbb{N}$. Assim, se tomarmos $n_{0}=\max \left\{j_{1}, j_{2}\right\}$ e teremos

$$
\bigcap_{n=1}^{n_{0}} C_{j}=\varnothing .
$$

Portanto $\Theta$ é uma classe compacta.

Definição 2.1.3. Una classe $\mathcal{E}$ de subconjuntos de um conjunto E é chamada uma semiálgebra se ela satisfaz as seguintes condições:

1) $\varnothing$ e E estão em $\mathcal{E}$;

2) $\mathcal{E}$ é fechada sobre intersecção finita;

3) se $S \in \mathcal{E}$, então $S^{c}$ é a uniäo de uma famain finita de subconjuntos disjuntos dois a dois de $\mathcal{E}$.

Considere a classe $\Delta$ formada por intersecção finita de elementos de $\Theta$ união com o $\varnothing$ e $\Omega$,

$$
\Delta=\left\{C: C=B_{1} \cap \ldots \cap B_{n}, \quad B_{i} \in \Theta, \quad n \in \mathbb{N}\right\} \cup\{\varnothing\} \cup\{\Omega\} .
$$

Lema 2.1.4. A classe $\Delta$ é uma semi-álgebra compacta.

Dem: Desde que $\Delta$ é formada via intersecção finita de elementos de uma classe compacta $(\Theta)$, obtemos que $\Delta$ também é compacta, Neveu $[1965$, lema I.6.1, pg.26]. Só falta mostrar que $\Delta$ é uma semi-álgebra. Por construção $\varnothing$ e $\Omega$ estão contidos em $\Delta$. Considere agora dois elementos $C_{1}$ e $C_{2}$ de $\Delta, C_{1}=B_{1}^{1} \cap \ldots \cap B_{k_{1}}^{1}$ e $C_{2}=B_{1}^{2} \cap \ldots \cap B_{k_{2}}^{2}$, assim

$$
C_{1} \cap C_{2}=B_{1}^{1} \cap B_{1}^{2} \cap \ldots \cap B_{k_{1}}^{1} \cap B_{k_{2}}^{2}
$$

É a intersecção finita de elementos de $\Theta$ pois cada $B_{j}^{i} \in \Theta$, portanto $C_{1} \cap C_{2} \in \Theta$. Seja $A \in \Delta$ então, $A=B_{1} \cap \ldots \cap B_{k}, B_{i} \in \Theta$. Assim $A^{c}-\cup\left[B_{1}^{\prime} \cup \ldots \cup B_{k}^{\prime}\right]$, onde $B_{\jmath}^{\prime}=B_{j}$ ou $B_{j}^{\prime}=B_{j}^{c}$ e a união é tomada sobre todas as possíveis combinações de $B_{j}^{\prime}$, exceto $B_{j}^{\prime}=B_{j}, \forall j=1, \ldots, n$. Desde que todos os termos da união são disjuntos dois a dois, concluímos que $\Delta$ é uma semi-álgebra. $\square$ A partir da semi-álgebra compacta $\Delta$ podemos construir uma álgebra compacta de forma trivial.

Lema 2.1.5. A classe

$$
\Upsilon=\left\{A: A-B_{1} \cup \ldots \cup B_{n}, B_{i} \cap B_{j}=\varnothing(i \neq j), n \in \mathbb{N}, B_{i} \in \Delta\right\}
$$

é uma álgebra. 
Dem: Desde que $\Upsilon$ é formada por união finita disjunta de elementos da classe compacta $\Delta$, obtemos que $\Upsilon$ também é compacta, ver Neveu(1965, lema I.6.1, pg. 26). Agora, vamos provar que $\Upsilon$ é uma álgebra. Para isto, basta mostrarmos que a classe $\Upsilon$ é fechada. por interseç̧ão finita e pcla operação de complementar. Considere $A_{1}$ e $A_{2}$ dois elementos em $\Upsilon$. Hutão temos que

$$
A_{i}=\bigcup_{j=1}^{n_{i}} B_{j}^{i} \quad, \quad i=1,2
$$

onde $\left\{B_{j}^{i}: j=1, \ldots n_{i}, i=1,2\right\}$ é uma família de elementos de $\Delta$, tal que

$$
B_{j}^{i} \cap B_{l}^{i}=\varnothing \quad(j \neq l), \quad i-1,2
$$

Fintão, temos que

$$
A_{1} \cap A_{2}=\bigcup_{j=1}^{n_{1}} \bigcup_{l=1}^{n_{2}}\left(B_{j}^{1} \cap B_{l}^{2}\right)
$$

Desde que os elementos da união são disjuntos dois a dois, concluímos que $\Upsilon$ é fechada por intersecção finita. Para finalizar vamos mostrar que $\Upsilon$ é fechada por operaçāo de complementar. Seja $A \in \Upsilon$, então existe $B_{1}, \ldots, B_{n}$ pertencentes a $\Delta$ com $B_{i} \cap B_{j}=$ $\varnothing:(i \neq j)$,

$$
A=\bigcup_{j=1}^{n} B_{j} .
$$

Então, temos que

$$
A^{c}=\bigcap_{j=-1}^{n_{t}} B_{j}^{\mathrm{c}} .
$$

Descle que $B_{j} \in \Delta$ obtcmos que $B_{j}^{c} \in \Upsilon$. Desta forma, concluímos que $A^{c} \in \Upsilon$. Portanto $\Upsilon$ é umáálgebra.

๑. A classe $\Upsilon$ é chamada álgebra dos cilindros.

Definição 2.1.4. Um espaço mensurável $(E, \mathcal{E})$ é separável se existe uma sequência $\left\{E_{n}\right\}$ que gera a $\sigma$-álgebra, isto é, $\mathcal{E}=\sigma\left\{E_{n}\right\}$

Definição 2.1.5. Um espaço mensurável separável $(E, \mathcal{E})$ é IIausdorff se existe uma sequência $\left\{E_{n}\right\} \subset \mathcal{E}$ que separa pontos em $(E, \mathcal{E})$, isto é, para cada $x_{1}$ e $x_{2} \in E$ distintos, existe um $m \in \mathbb{N}$ tal que $I_{\left\{E_{m}\right\}}\left(x_{1}\right) \neq I_{\left\{E_{m}\right\}}\left(x_{2}\right)$.

Considere $\Im$ a $\sigma$-álgebra gerada pela álgebra dos cilindros $\Upsilon$. Tendo $\Omega$ e $\Im$ obtemos um espaço mensurável $(\Omega, \Im)$ que é separável e Hausdorff, pois a álgebra $\Upsilon$ é enumerável e separa pontos $\mathrm{em}(\Omega, \Im)$. Vamos definir agora uma função de conjunto sobre a álgebra $\Upsilon$. Dada uma função de conjunto $\lambda: \Upsilon \longrightarrow[0,1]$ satisfazendo 
a) $\lambda(\varnothing)=0 ; \lambda(\Omega)=1$

b) se $A_{1}, A_{2} \in \Upsilon \operatorname{com} A_{1} \cap A_{2}=\varnothing$, então

$$
\lambda\left(A_{1} \cup A_{2}\right)=\lambda\left(A_{1}\right)+\lambda\left(A_{2}\right),
$$

dizemos que $\lambda$ é uma função de conjunto finitamente aditiva sobre a álgebra dos cilindros $\Upsilon$.

Lema 2.1.6. Toda funçäo de conjunto $\lambda: \Upsilon \longrightarrow[0,1]$ finitamente aditiva é $\sigma$-aditiva.

Dem: Desde que $\Upsilon$ é uma álgebra, é suficiente mostrarmos que $\lambda$ é contínua no vazio. Seja $\left\{C_{n}\right\} \subset \Upsilon$ uma sequência tal que $C_{n} \supset C_{n-1}$ ○ $\bigcap_{n=1}^{\infty} C_{n}=\varnothing$, vamos mostrar que

$$
\lim _{n} \lambda\left[C_{n}\right]=0
$$

mas $\Upsilon$ é uma classe compacta, nesse caso existe $n_{0} \in \mathbb{N}$, satisfazendo $\bigcap_{n=1}^{n_{n}} C_{n}=\varnothing$, cntão $\lambda\left[C_{n}\right]=\lambda\left[\bigcap_{k=1}^{n} C_{k}\right] \leq \lambda\left[\bigcap_{k=1}^{n_{0}} C_{k}\right]=\lambda[\varnothing]=0, \forall n \geq n_{0}$.

Portanto, quando $C_{n} \downarrow \varnothing$ nós temos que $\lambda\left[C_{n}\right]$ i 0 .

Considere $\Gamma$ a classe formada por intersecção enumerável de elementos de $\Upsilon$, isto é,

$$
\Gamma=\left\{A: A=B_{1} \cap B_{2} \cap B_{3} \cap \ldots=\bigcap_{n=1}^{\infty} B_{n} ; B_{i} \in \Upsilon, i \in \mathbb{N}\right\}
$$

Desde que $\Upsilon$ é umá álgebra, podemos formar elementos $D_{n}=B_{1} \cap B_{2} \cap \ldots \cap B_{n}$ satisfazendo

a) $D_{n} \in \Upsilon ; \forall n \in \mathbb{N}$

b) $D_{n} \supset D_{n+1} ; \forall n \in \mathbb{N}$;

c) $\bigcap_{n=1}^{\infty} B_{n}=\bigcap_{n=1}^{\infty} D_{n}=A$

Desta forma, os elementos de $\Gamma$ podem ser representados como intersecção enumerávol de sequências monótonas decrescentes de elementos de $\Upsilon$.

Lema 2.1.7. Considere $\lambda: \Upsilon \longrightarrow[0,1]$ uma funçăo de conjunto finitamente aditiva. Então, existe uma única probabilidade $P$ sobre $(\Omega, \Im)$, tal que

a.) $P[B]-\lambda[B] ; \forall B \in \Upsilon$; 
b) Para todo $K \in \Gamma$, existe uma sequência $\left\{E_{n}\right\} \subset \Upsilon \operatorname{com} E_{n} \supset E_{n+1} e \bigcap_{n=1}^{\infty} E_{n}=K$. Além disso, temos que

$$
P[K]=\lim _{n \rightarrow \infty} \lambda\left[E_{n}^{i}\right]
$$

c) Para $A \in \Im$ temos que

$$
P[A]=\sup \{P[K]: K \subset A, K \in \Gamma\}
$$

Além disso, a classe $\Gamma$ é compacta.

Dem: Desde quo I' é formarla por interseçãa enumerável de elementos da álgebra compacta $\Upsilon$, obtemos que $\Gamma$ também é uma classe compacta, ver Neveu [1965, lema I.6.1, pg. 26] Como consequência do lema 2.1.6 temos que a função de conjunto $\lambda$ é $\sigma$ aditiva. Então, obtemos do teorcma da extensão de Carathéodory, que cxiste uma única probabilidade $P$ sobre $(\Omega, \Im)$ satisfazendo as propricedades $(a), b)$ e $c)$.

A seguir, vamos mostrar que $(\Omega, \Im)$ é um espaço de Radon, conforme introduzido cm Leão, Fragoso, Ruffino (1999). Esta classe de espaços mensuráveis é importante para garantir a existência de probabilidade condicional regular, que serão utilizadas para garantir uma relaçào de existência e unicidade entre um processo previsível crescente e o processo pontual.

Teorema 2.1.1. O espaço mensurável $(\Omega, \Im)$ é Radon, isto é, para toda probabilidade $P$ sobre $(\Omega, \Im)$, temos que.

$$
P[A]=\sup \{P[K]: K \subset A, K \in \Gamma\}
$$

onde $\Gamma$ é uma classe compacta.

Prova: Basta observarmos que toda probabilidade $P$ sobre $(\Omega, \Im)$ é uma função de conjunto $P: \Upsilon \longrightarrow[0,1]$ finitamente aditiva, e aplicamos o lema 2.1.7.

\subsection{Processo Pontual}

Nesta seção vamos estudar probabilidades sobre $(\Omega, \Im)$ e o processo estocástico associado. Para isto, começamos estudando filtrações sobre este espaço mensurável.

Definição 2.2.1. Uma familia de sub-o-álgebras $\left\{\Im_{t}, t \geq 0\right\}$ de uma $\sigma$-álgebra $\mathcal{F}$ é chamada crescente se $s \leq t$ implica $\mathcal{F}_{s} \subset \mathcal{F}_{\ell}$. Uma famitia crescente de sub- $\sigma$-álgebras é chamada uma filtraçăo. 
Definição 2.2.2. Quando $\left\{\Im_{t,}, t \geq 0\right\}$ é uma filltração, a $\sigma$-álgebra $\bigcap_{h>0} \Im_{t+h}$ é denotada. por $\Im_{+1}$. O limite à esquerda $\Im_{t-}$ é a menor $\sigma$-álgebra contendo todos os conjuntos em $\bigcup_{h>0} \Im_{t-h}$ e é escrito como $\sigma\left\{\bigcup_{h>0} \Im_{t-h}\right\}$

Definiçāo 2.2.3. Uma filtraçăo é contínua à direita se $\Im_{t+}=\Im_{t}$.

Jefinimos uma filtração sobre o espaço do trajetórias $\Omega$ por

$$
\begin{aligned}
\Im_{0} & =\{\varnothing, \Omega\} ; \\
\Im_{t} & =\sigma\left\{C\left[k ; n ; w_{n}\right]: w_{n} \in S, k \in \mathbb{Q} \cap[0, t], n \in \mathbb{N}\right\}, \quad \iota \in[0, \infty) \\
\Im_{\infty} & =\sigma\left\{\bigcup_{t \in[0, \infty]} \Im_{t}\right\}-\Im .
\end{aligned}
$$

A seguir, vamos mostrar que a filtração é contínua à direita.

Lema 2.2.1. Temos que

$$
\Im_{t}=\left\{A \in \Im: \text { se } x \in A \text { e } x^{\prime}(s)=x(s), s \leq t \text {, então } x^{\prime} \in A\right\}
$$

Além disso, temos que $\left\{\Im_{t}\right\}$ é contínua à direita.

Prova: Denotamos por

$$
\begin{aligned}
G_{0} & =\Im_{0} \\
G_{t} & =\left\{A \in \Im: \text { se } x \in A \text { e } x^{\prime}(s)=x(s), s \leq t, \text { então } x^{\prime} \in A\right\}
\end{aligned}
$$

Temos que mostrar que $\Im_{t}=G_{t}, t \in[0, \infty)$. Provemos que $\Im_{t} \subset G_{t}$. Para isto, basta mostrarmos que todo cilindro $C\left[k ; n ; w_{n}\right], w_{n} \in S, k \in \mathbb{Q} \cap[0, t], n \in \mathbb{N}$, pertence at $G_{t}$. Temos que,

$$
C\left[k ; n ; w_{n}\right]:\left\{x \in \Omega: x(k) \subset \pi_{n}^{-1}\left(\left\{w_{n}\right\}\right)\right\} .
$$

Assim, se $x \in C\left[k ; n ; w_{n}\right]$ e $x^{\prime}(s)-x(s), s \leq t$, cutão

$$
x^{\prime}(s) \in \pi_{n}^{-1}\left(\left\{u_{n}\right\}\right)
$$

para todo $k \in \mathbb{Q} \cap[0, t], n \in \mathbb{N}$ e $w_{n} \in S$. Desta forma, obtemos que $\Im_{t} \subset G_{t}$. Agora vamos mostrar a volta, isto é, que $\Im_{t} \supset G_{t}, t \in[0, \infty)$. Se $A \in G_{l}$ tomos que

$$
A \nsubseteq C\left[k ; n ; w_{n}\right]
$$


para todo racional $k>t, n \in \mathbb{N} e w_{n} \in S$. Se $A=\Omega$ nada temos a fazer. Considere $A \subset \Omega$ e $A \neq \Omega$, existem elementos $s \in[0, t], n_{0} \in \mathbb{N}$ e $w_{u_{0}} \in S$ tais que

$$
A \subset C\left[s ; n_{0} ; w_{n_{0}}\right]=\left\{x \in \Omega: x(s) \in \pi_{n_{0}}^{-1}\left(\left\{w_{n_{0}}\right\}\right)\right\}
$$

Como $\mathbb{Q} \cap[0, t]$ é denso en $[0, t]$, existe uma seqüência $\left\{k_{j}\right\} \subset \mathbb{Q} \cap[0, t]$ tal que $k_{n} \downarrow s \quad\left(k_{j} \geq k_{j+1} \geq s\right)$. Então, utilizando a propriedade de contimuidade à direita das funçöes indicadoras $I_{\left.\left\{i_{j}, \infty\right)\right\}}($.$) , concluímos que$

$$
C\left[s ; n_{0} ; w_{n_{0}}\right]=\bigcap_{j=1}^{\infty} C\left[k_{j} ; n_{0} ; w_{n_{0}}\right] \in \Im_{i} .
$$

Desde: que $A \in \Im$, obtemos que

$$
A \in \sigma\left\{C\left[k ; n ; w_{n}\right]: k \in \mathbb{Q} \cap[0, t], n \in \mathbb{N}, w_{n} \in S\right\}=\Im_{t}, t \in[0, \infty)
$$

Desta forma conchúmos que $G_{t} \subset \Im_{t}$. Considere, $A \in \Im_{t+.}$ Seja $x \in A$ e $x^{\prime} \in \Omega$ tal que $x(s)=x^{\prime}(s)$ para $s \leq t$. Usando a continuidade das funçoes indicadoras, temos que $x^{\prime} \in A$ e $A \in \Im_{t}$ pois do resulatalo anterior $G_{t}=\Im_{t}$. Dessa forma $\Im_{t+} \subset \Im_{t}$, portanto $s_{t-}-S_{l}$.

A seguir, apresentamos algumas definições que estaremos utilizando durante o trabalho.

Definição 2.2.4. Uma variável aleatória $T: \Omega \rightarrow[0, \infty]$ é denorninada tempo de parada se $\{x: T(x) \leq t\} \in S_{t}$ para todo $t \in[0, \infty]$

Para todo $x \in \Omega$, temos $0=t_{0}<t_{1}<\ldots<t_{k} \in[0, \infty)$ corn $k \in \mathbb{N} e$

$$
\begin{aligned}
& x(0)=(0,0,0,0,0, \ldots), \\
& x(s)=\left(I_{\left.\left\{! t_{1}, \infty\right)\right\}}(s), I_{\left\{\left[t_{2}, \infty\right)\right\}}(s), \ldots, I_{\left\{\left[t_{k}, \infty\right)\right\}}(s), 0,0,0,0, \ldots\right), s>0 .
\end{aligned}
$$

Assim, definimos uma função $T_{j}: \Omega \rightarrow[0, \infty]$ satisfazendo

$$
\begin{aligned}
T_{j}(x) & =\inf \{s \in[0, \infty]: x(s)=\underbrace{(1,1,1, \ldots, 1}_{j \text { vezes }}, 0,0, \ldots)= \\
& =t_{j} ; \text { se } j \leq k \\
& =+\infty ; \text { se } j>k
\end{aligned}
$$

Teorema 2.2.1. A funçäo $T_{j}: \Omega \longrightarrow[0, \infty]$ é um tempo de parada com respeito à filtraçäo $\Im_{t}$. 
Prova: Para todo $t \in[0, \infty]$, temos que

$$
\begin{aligned}
\left\{x \in \Omega: T_{j}(x)=0\right\} & =\varnothing \\
\left\{x \in \Omega: T_{j}(x) \leq+\infty\right\} & =\Omega \\
\left\{x \in \Omega: T_{j}(x) \leq t\right\} & =\left\{x \in \Omega: 0=t_{0}<t_{1}<\ldots<t_{k}, \quad l_{j} \leq t, k \geq j .\right\}
\end{aligned}
$$

Seja $k_{i} \in \mathbb{Q} \operatorname{com}\left\{k_{i}\right\} \downarrow t$, então

$$
C\left[k_{i} ; ; 1\right]=\left\{x \in \Omega: x\left(k_{i}\right) \in \pi_{l}^{-2}(\{1\})\right\} \in \Im_{k_{z}}
$$

Além disso, temos que

$$
\left\{x \in \Omega: T_{j}(x) \leq k_{i}\right\}=C\left[k_{i} ; 1 ; 1\right] \cap C\left[k_{i} ; 2 ; 1\right] \cap C\left[k_{i} ; 3 ; 1\right] \cap \ldots \cap C\left[k_{i} ; j ; 1\right] \in \Im_{k_{i}}
$$

Desde que $\left\{\Im_{t}\right\}$ é contínua à direita, concluímos que

$$
\left\{x \in \Omega: T_{j}(x) \leq t\right\}=\bigcap_{i=1}^{\infty}\left\{x \in \Omega: T_{j}(x) \leq k_{i}\right\} \in \Im_{t+}=\Im_{t} .
$$

Portanto, $T_{j}: \Omega \longrightarrow[0, \infty]$ é um tempo de parada.

Com isso obtemos uma sequência de tempos de parada $\left\{T_{j}\right\}$ satisfazendo

1) $T_{j}(x)>0 ; \forall x \in \Omega$;

2) $T_{j}(x)>T_{j+1}(x) ; \forall x$ t.q. $T_{j}(x)<\infty$;

3) $T_{j}(x)=T_{j+?}(x) ; \forall x$ t.q. $T_{j}(x)-\infty$

Dados os tempos de parada $\left\{T_{j}\right\}$ podemos definir uma função de contagem $N:[0, \infty) \times \Omega \rightarrow \mathbb{N} \cup\{\infty\}$, por

$$
N_{t}(x)=\sum_{j=1}^{\infty} I_{\left\{T_{j} \leq t\right\}}(x), x \in \Omega, t \in[0, \infty),
$$

Fsta função é crescente cm t, tem saltos unitários e admite limite à direita em $t$ para todo $x \in \Omega$. Para mostrarmos que a função $N_{t}$ é crescentee em t, scjam $j \in \mathbb{N}$ e $t_{1}<t_{2} \in \mathbb{R}$, assim

$$
I_{\left\{T_{j}(x) \leq t_{1}\right\}}(x) \leq I_{\left\{T_{j}(x) \leq t_{2}\right\}}(x) \quad \forall x \in \Omega .
$$

Então a função de contagem satisfä

$$
N_{t_{1}}(x) \leq N_{t_{2}}(x) \quad \forall x \in \Omega
$$


Além disso, segne do teorema da convergência dominada de Lebesgue, que

$$
\lim _{h \rightarrow 0} N_{t}(x)=\lim _{h \rightarrow 0} \sum_{j=1}^{\infty} I_{\left\{T_{j} \leq t, h\right\}}(x)=\sum_{j=1}^{\infty} I_{\left\{T_{j} \leq t\right\}}=N_{t}(x), \quad x \in \Omega .
$$

Assim, $N_{t}$ é contínua à direita e do mesmo teorema segue que $N_{t}$ tem limite à esquerda

$$
\lim _{h \rightarrow 0} N_{t+h}(x)=\lim _{h \rightarrow 0} \sum_{j=1}^{\infty} I_{\left\{T_{j} \leq t+h\right\}}(x)=N_{t \vdash}(x)<+\infty, x \in \Omega .
$$

Dado $N_{t}$, também podemos encontrar os tempos de parada, basta tomar $T_{j}=\inf \left\{t: N_{t}-j\right\}$. Na secquencia vamos introduzir uma probabilidade sobre $(\Omega, \Im)$ e estudar as propriedades dos tempos de parada.

Definiçāo 2.2.5. Dado uma probubilidade $P$ sobre $(\Omega, \Im)$, dizemos que o processo estocástico $N=\left(N_{t}, \Im_{t}, \Im, F^{\prime}\right)$ é um processo pontual.

O processo pontual será investigado mais profundamente quando utilizarmos alguns resultados importantes da teoria martingale, a decomposição de Doob-Meyer e os teoremas de existencia e unicidade do compensador.

A seguir apresentamos alguns exemplos de processos pontuais que estarão servindo de ilustraçäo para alguns resultados no decorrer desse capítulo, o processo de Poisson homogenen e não-homogèneo.

Exemplo 2.2.1. (Processo de Poisson Homogêrueo)

O processo de Poisson $\left\{N_{t}: t \geq 0\right\}$ pode ser encontrado em muitas aplicaçôes, ele é usado para modelar processos de chegada, como número de clientes que chegam em um caixa cletrônico, enissaio de partículas radioativas, número de chamadas telefônicas que chegam num determinado intervalo de tempo etc. Considere $T_{1}, \ldots, T_{k}$ os tempos de chegada sucessivos em um processo de Poisson definido sobre um espaço de probabilidade $(\Omega, \Im, P)$, então $T_{1}, T_{2}-T_{1}, \ldots, T_{k}-T_{k-1}$ são independentes e identicamente distribuídos como uma distribuição exponencial com parâmetro $1 / \lambda$ e a probabilidade induzida por $\left\{N_{t}: t \geq 0\right\}$ é a distribuiçăo de Poisson com parâmetro $\lambda t$,

$$
P\left[N_{t}=k\right]=\frac{\exp ^{-\lambda t}(\lambda t)^{k}}{k !}
$$

segue daí, que o valor esperado de $\left\{N_{t}: t \geq 0\right\}$ é dado por $E\left[N_{t}\right]=\lambda t$ e a variància por $\operatorname{var}\left[N_{t}\right] \cdots \lambda t$. O parânetro $\lambda$ é chamado de intensidade ou taxa de falha do processo e no caso de $\lambda$ ser uma função de $t$, teremos o processo de Poisson não-homogêneo. Quando 
ele for igual a um, diremos processo de Poisson com parâmetro unitário. Para o processo de Poisson homogêneo temos que, para qualquer $t, s \geq 0, N_{s+t} \cdots N_{s}$ é independento de $\left\{N_{u}: u \leq t\right\}$, isto é, o processo de Poisson tem incrementos independentes e para qualquer $t, s \geq 0$, a distribuição de $N_{s+t} \cdot N_{s}$ é independente de t, isto é, o processo de Poisson tem incrementos estacionários. As trajetórias $t \rightarrow N_{t}$ do processo são funçōes escada, contínuas à direita, crescentes com saltos unitários e $N_{0}=0$. A deusidade de probabilidade do n-ésimo tempo $T_{n}$ de chegada do processo é uma Gama $(n, \lambda(t))$ dada por

$$
f_{T_{n}}(t)=\lambda e^{-\lambda t} \frac{(\lambda t)^{n-1}}{(n-1) !}
$$

descle que $P\left[T_{n} \leq t\right]-P\left[N_{t} \geq n\right]$.

Exemplo 2.2.2. (Processo de Poisson Nẫo-Homogêneo)

O processo de Poisson $\left\{N_{t}: t \geq 0\right\}$ definido em um espaço de probabilidade $(\Omega, \Im, P)$ é um processo de Poisson não-homogêneo quando os incrementos não são estacionários, isto é, para qualquer $t, s \geq 0$, a distribuç̧̃o de $N_{s+t}-N_{s}$ não é independente de t. Nesse caso a taxa de falha do processo para $N_{t}$ ć dada por uma função determinística $\lambda(t)$ e a taxa de falha acumulada é $A(t)=\int_{0}^{t} \lambda(s) d s$. A distribuição de probabilidade de $N_{t} e^{\prime}$ uma distribuição de Poisson com parâmetro $A(t)=\int_{0}^{t} \lambda(s) d s$.

$$
\begin{aligned}
P\left[N_{t}=k\right] & =\exp \left\{-\int_{0}^{t} \lambda(s) d s\right\} \cdot \frac{\left\{\cdot \int_{0}^{t} \lambda(s) d s\right\}^{k}}{k !}-\frac{\{A(t)\}^{k}}{k !} \\
& =\exp \{-A(t)\} \frac{\{}{k}
\end{aligned}
$$

$\Lambda$ densidade de probabilidade do n-ésimo tempo $T_{n}$ de chegada do processo é dada por

$$
f_{T_{n}}(t)-\lambda(t) \exp \left\{-\int_{0}^{t} \lambda(s) d s\right\} \frac{\left(\int_{0}^{t} \lambda(s) d \cdot s\right)^{n \cdots 1}}{(n-1) !} .
$$

\subsection{Decomposição de Doob-Meyer}

A decomposição de Doob-Meyer é um resultado fundamental na teoria des processos estocásticos, ele garante que un processo pontual $N_{t}$ pode ser decomposto como a soma de um martingale com um dado processo, chamado compensador. Para prosseguirmos, daremos algumas explicaçós e definições de alguns termos que serão essenciais e que serão bastante utilizados. 
Definição 2.3.1. Um processo estocástico $\left\{X_{t}, t \geq 0\right\}$ é adaptado para uma filtração se, para cada $t \geq 0, X_{t}$ é $\Im_{t}$-mensurável.

Definição 2.3.2. Seja $X=\left\{X_{t}: t \geq 0\right\}$ um processo estocástico contínuo à direita com limites à esquerda e $\left\{\Im_{t}: t \geq 0\right\}$ uma filtração, definida num espaço de probabilidade $(\Omega, \Im, P) . X$ é chamado um martingale com respeito à $\left\{\Im_{t}: t \geq 0\right\}$ se

1) $X$ é udaptado para $\left\{\Im_{t}: t \geq 0\right\}$,

2) $\left.E \mid X_{t}\right\}<\infty$ para todo $t<\infty$,

3) $E\left[X_{\ell+s} \mid \Im_{l}\right]=X_{l} \quad(P-q . c)$ para $s \geq 0$ et $\geq 0$.

$X$ será chamado um submartingale se 3) for trocado por

$$
E\left[X_{t+s} \mid \Im_{t}\right] \geq X_{t} \quad(P-q . c .)
$$

e um supermartingale se 3) for trocado por

$$
E\left[X_{t+s} \mid \Im_{t}\right] \leq X_{t} \quad\left(f^{\prime}-q . c .\right)
$$

O processo $N_{t}$ definido em 2.1 é um submartingale. O primeiro item de 2.3 .2 verificamos do fato que cada tempo de parada $T_{j}$ é $S_{t}$-mensurável, isto é, $\left\{x \in \Omega: T_{j}(x) \leq t\right\}$ c $\Im_{t}$, a função $\left.I_{\{x \in \Omega} \Omega: T_{j}(x) \leq t\right\}$ á $\Im_{t}$-mensurável e a soma de funções mensuráveis é mensurável. Para verificar o segundo item de 2.3 .2 basta verificar que para todo intervalo finito o processo $N_{t}$ salta somente un número finito de vezes, portanto é integrável para todo $t<\infty$. O terceiro item de 2.3 .2 segue de

$$
\begin{aligned}
E\left[N_{t} \mid \Im_{s}\right] & =E\left[N_{t}+N_{s}-N_{s} \mid \Im_{s}\right]=E\left[N_{t}-N_{s} \mid \Im_{s}\right]+E\left[N_{s} \mid \Im_{s}\right] \\
& =E\left[N_{t}-N_{s} \mid \Im_{s}\right]+N_{s} \geq N_{s}, \quad t \geq s, \quad(P-q . c .)
\end{aligned}
$$

pois $N_{t}$ é uma função crescente. Assim, $N_{t}$ é um submartingale e segue desse resultado que o processo de Poisson também é um submartingale.

Definição 2.3.3. Scja $(\Omega, \Im, P)$ um espaço de probabilidade com uma filtração $\left\{\varsigma_{t}: t \geq 0\right\} . A$ o-ályebra $\mathrm{em}[0, \infty) \times \Omega$ gerada por todos os conjuntos da forma

$$
[0] \times A, \quad A \subset \Im_{1},
$$

$$
(a, b] \times A, \quad 0 \leq a<b<\infty, A \in \Im_{a},
$$

é chamada a $\sigma$-álgebra previsivel para a filtração $\left\{\Im_{t}: t \geq 0\right\}$ e será denotada por $\Im^{p}$. 
Definição 2.3.4. Um processo $X$ é chamado previsivel com respeito a uma filtraçäo $\Im_{t}$ se, como uma aplicação de $[0, \infty) \times \Omega$ em $R$, ele é mensurável com respeito a $\sigma$-álgebra previsivel. Nós chamamos $X$ de um processo $\Im_{t}$-previsível.

A previsibilidade de um processo implica que nós podemos prever valores em qualquer ponto dado os valores em pontos precedentes como no caso de processos que são contínuos à esquerda. Qualquer processo com trajetórias contínuas quase-certamenté é previsivel Por exemplo, o processo de Wiencr é um processo previsível enquanto que o processo do Poisson não é, pois suas trajetórias são do tipo escada. [B.L.S. Prakasa Rao(1987)]

Definição 2.3.5. Um tempo de parada $\theta$ (com respeito à filtração $\Im_{t}, t \geq 0$ )é chamado previsivel se um processo pontual $N_{t}=I_{\{\theta \leq t\}}$ é previsivel.

De Liptser e Shiryayev [1978, pg. 239] um tempo de parada $\theta$ é previsível se e soment. se existe uma sequência crescente de tempos de parada $\left\{\theta_{n}, n \geq 1\right\}$, tais que $\theta_{n}<\theta$ e $\lim _{n} \theta_{n}=\theta(P-q . c)$.

Definição 2.3.6. Um tempo de parada $\sigma$ (com respeito à filtração $\Im_{t}, t \geq 0$ ) é dito ser totalmente inacessivel se $P[\theta=\sigma<\infty]=0$ para cada tempo de parada $\theta$ prenisível.

Definição 2.3.7. Um processo estocástico $X=\left\{X_{t}: \iota \geq 0\right\}$ é quadrado intergrável se $\sup _{t} E\left|M_{t}\right|^{2}<\infty, t \geq 0$.

Considere um martingale $M$ quadrado integrável sobre $\left(\Omega, \Im_{l}, \Im, P\right)$ então,

$$
E\left[M_{t}^{2} \mid \Im_{s}\right] \leq\left(E\left[M_{t} \mid \Im_{s}\right]\right)^{2}=M_{s}^{2}, t>s
$$

o sinal de desigualdade é garantido pela desigualdade de Jensen. Dessa forma, $M^{2}$ ó um submartingale e segue da decomposição de Doob-Meyer a seguinte definição,

Definição 2.3.8. A variação quadrática previsível de um martingale $M$ quadrado integrável é um processo previsivel crescentc contínuo à direita $\langle M, M\rangle$ tal que.

$$
M=M^{2}-<M, M>
$$

é um martingale.

Teorema 2.3.1. Supondo que $E\left[N_{t}\right]<\infty, t \in[0, \infty)$, entäo, existe um único (no sentido estocástico) processo crescente previsível $A_{t}$, adaptado para $\varsigma_{\text {s }}$ com $A_{0}=0$, tal que:

$$
M=N-A
$$


onde $M$ é um martingale quadrado integrável e,

$$
<M, M>=<M>-A,
$$

também, o compensador $A_{t}, t \geq 0$, é contínuo $t<\infty(P-q . c)$ se e somente se os tempos de salto do processo $N_{t}, t \geq 0$, são totalmente inacessiveis.

Prova $O$ processo $N_{t}$ é um submartingale com trajetórias contínuas à direita e portanto de classe DL (ver [Teorema 3.7, pag.62, Lipster e Shyriayev]) e da decomposição de Doob-Meyer [Liptser e Shiryayev Teorema 3.8, pag. 65]

$$
M=N-A
$$

é um martingale. Agora precisamos mostrar que $M$ é quadrado integrável, portanto uniformemente integrável. Considercmos os seguintes instantes de salto $T_{n}: n=1,2, \ldots$ de $\mathrm{N}$, totalmente inacessíveis. Dado que o processo $\left(N_{t \wedge T_{n}}, \Im_{t}\right)$ é quadrado integrável, pois cle é não-decrescente e limitado, isto é,

$P\left[0<N_{t \wedge T_{n}} \leq n_{\mathrm{j}}^{\prime}=1\right.$, para todo t. Assim, (ver [Meyer: Probabilités et Potenticl, seção VIII, teorema 31]),

$$
M_{t \wedge T_{n}}=N_{t \wedge T_{n}}-A_{t \wedge T_{n}}
$$

ó martingale quadrado integrável. Agora precisamos mostrar que:

$$
A_{t \wedge T_{n}}-<M, M>_{t \wedge T_{n}}
$$

que é o mesmo que mostrar

$$
M_{t \wedge T_{t}}^{2}-A_{t \wedge T_{n}}
$$

ć um martingale. Assim, para qualquer $\epsilon>0$ e $s<t$ arbitrário, define-se uma sequência $S_{0} \leq S_{1} \leq S_{2} \leq \ldots$ de tempos de parada, tal que

$$
\begin{gathered}
S_{0}=s \quad, \quad \lim _{k} S_{k}=t \\
0 \leq A_{t \wedge T_{n} \wedge S_{k}}-A_{t \wedge T_{n} \wedge S_{k-1}} \leq \epsilon(P-q . c .) \\
0 \leq N_{t \wedge T_{n} \wedge S_{k}}-N_{t \wedge T_{n} \wedge S_{k-1}} \leq \epsilon(P-q . c .)
\end{gathered}
$$

A existência da sequência está garantida pela continuidade do compensador. Então,

$$
\sum_{k=1}^{\infty}\left[M_{i \wedge T_{n} \wedge S_{k}}-M_{t \wedge T_{n} \wedge S_{k-1}}\right]^{2}=
$$




$$
\begin{gathered}
\sum_{k=1}^{\infty}\left[N_{t \wedge T_{n} \wedge S_{k}}-N_{t \wedge T_{n} \wedge S_{k-1}}-A_{t \wedge T_{n} \wedge S_{k}}+A_{\left.t \wedge T_{n} \wedge S_{k-1}\right]^{2}=}\right. \\
\sum_{k=1}^{\infty}\left[N_{\left.t \wedge T_{n} \wedge S_{k}-N_{t \wedge T_{n} \wedge S_{k-1}}\right]^{2--}}\right. \\
-2 \sum_{k=1}^{\infty}\left[N_{t \wedge T_{n} \wedge S_{k}}-N_{\left.t \wedge T_{n} \wedge S_{k-1}\right]}\right]\left[A_{t \wedge T_{n} \wedge S_{k}}-A_{t \wedge T_{n} \wedge S_{k-1}}\right]+ \\
+\sum_{k=1}^{\infty}\left[A_{t \wedge T_{n} \wedge S_{k}}-A_{t \wedge T_{n} \wedge S_{k-1}}\right]
\end{gathered}
$$

Como, $\left(N_{t \wedge T_{n} \wedge S_{k}}-N_{l \wedge T_{n} \wedge S_{k-1}}\right)$ só pode assumir os valores f) ou 1, temos que

$$
\begin{gathered}
\sum_{k=1}^{\infty}\left[N_{t \wedge T_{n} \wedge S_{k}}-N_{t \wedge T_{n} \wedge S_{k-1}}\right]^{2}= \\
\lim _{m} \sum_{k=1}^{m}\left[N_{t \wedge T_{n} \wedge S_{k}}-N_{\left.t \wedge T_{n} \wedge S_{k-1}\right]=N_{t \wedge T_{n}}-N_{s \wedge T_{n}}}\right.
\end{gathered}
$$

Então,

$$
\begin{aligned}
& \left|E\left[\sum_{k=1}^{\infty}\left[M_{t \wedge T_{n} \wedge S_{k}}--M_{t \wedge T_{n} \wedge S_{k-1}}\right]^{2}-\left[N_{t \wedge T_{n} \wedge S_{k}} \quad N_{t \wedge T_{n} \wedge S_{k-1}}\right] \mid \Im_{s}\right]\right| \leq \\
& \left|2 \sum_{k=1}^{\infty}\left[N_{t \wedge T_{n} \wedge S_{k}}-N_{t \wedge T_{n} \wedge S_{k} \cdot 1}\right]\left[A_{t \wedge T_{n} \wedge S_{k}}-A_{t \wedge T_{n} \wedge S_{k-1}}\right]\right|+ \\
& \left|\sum_{k=1}^{\infty}\left[A_{t \wedge T_{n} \wedge S_{k}}-A_{t \wedge T_{n} \wedge S_{k-1}}\right]^{2}\right|=
\end{aligned}
$$

usando 2.2, temos que o termo do lado direito da desigualdade fica

$$
\left.=2 \epsilon\left[N_{t \wedge T_{n}}-N_{t \wedge T_{n}}\right]+\epsilon \mid N_{t \wedge T_{n}} \cdots N_{t \wedge T_{n}}\right]
$$

e como $\epsilon$ é arbitrário,

$$
E\left[\sum_{k=1}^{\infty}\left(M_{t \wedge T_{n} \wedge S_{k}}-M_{t \wedge T_{n} \wedge S_{k-1}}\right)^{2}-\left(N_{t \wedge T_{n} \wedge S_{k}}-N_{t \wedge T_{n} \wedge S_{k-1}}\right) \mid \Im_{s}\right]=0
$$

Usando o fato de que

$$
\begin{gathered}
E\left[\left(M_{t \wedge T_{n} \wedge S_{k}}-M_{t \wedge T_{n} \wedge S_{k}, 1}\right)^{2} \mid \Im_{s}\right]= \\
E\left[M_{t \wedge T_{n} \wedge S_{k}}^{2}-M_{t \wedge T_{n} \wedge S_{k-1}}^{2} \mid \Im_{s}\right]
\end{gathered}
$$

e como,

$$
N_{t \wedge T_{n t}}-A_{t \wedge T_{n}}
$$


é um martingale segue que

$$
E\left[N_{t \wedge T_{n}}-N_{s \wedge T_{n}} \mid \Im_{s}\right]=E\left[A_{t \wedge T_{n}}-A_{s \wedge T_{n}} \mid \Im_{s}\right\}
$$

Daí,

$$
\begin{gathered}
E\left[\sum_{k=1}^{\infty}\left(M_{t \wedge T_{n} \wedge S_{k}}^{2}-M_{t \wedge T_{n} \wedge S_{k-1}}^{2}\right)-\left(A_{t \wedge T_{n} \wedge S_{k}}-A_{t \wedge T_{n} \wedge S_{k-1}}\right) \mid \Im_{s}\right]= \\
E\left[\lim _{m} \sum_{k=1}^{m}\left(M_{l \wedge T_{n} \wedge S_{k}}^{2}-M_{t \wedge T_{n} \wedge S_{k \sim 1}}^{2}\right)-\left(A_{t \wedge T_{n} \wedge S_{k}}-A_{t \wedge T_{n} \wedge S_{k-1}}\right) \mid \Im_{s}\right]= \\
=E\left[\left(M_{t \wedge T_{n}}^{2}-M_{s \wedge T_{n}}^{2}\right)-\left(A_{t \wedge T_{n}}-A_{s \wedge T_{n}}\right) \mid \Im_{s}\right]=0
\end{gathered}
$$

Portanto

$$
M_{t \wedge T_{n}}^{2}-A_{s \wedge T_{n}}
$$

é um martingale. Com isso conclui-se que

$$
A_{t \wedge Y_{n}^{\prime}}-<M, M>_{t \wedge T_{n}}
$$

Desde que $M_{t \wedge T_{n}}$ converge para $M_{t}$, segue do lema de Fatou que

$$
E\left[M_{t}^{2}\right] \leq \lim _{n \rightarrow \infty} E\left[N_{t \wedge T_{n}}\right]=E\left[N_{t}\right]
$$

E pelo teorema da convergencia dominada

$$
\begin{gathered}
E\left[M_{t}^{2} \mid \Im_{s}\right]=E\left[\lim _{n} M_{t \wedge T_{n}}^{2} \mid \Im_{s}\right]= \\
=\lim _{n} E\left[M_{t \wedge T_{n}}^{2} \mid \Im_{s}\right] \leq \lim _{n} M_{s \wedge T_{n}}^{2}=M_{s}^{2}
\end{gathered}
$$

Assim, $M^{2}$ é um submartingale positivo, pela decomposição de Doob-Meyer existe um único processo crescente previsível $B$, tal que

$$
M^{2}-B
$$

6 um martingale. Agora usando 2.6 segue que.

$$
B_{t}=\lim _{n} B_{t \wedge T_{n}}=\lim _{n} A_{t \wedge T_{n}}=A_{t}
$$

a prova da segunda parte segue de [Liptser, Shiryayev, coroláriol,cap.18]. Portanto a prova está concluída.

\section{Exemplo 2.3.1.}


Considere o processo de Poisson homogêneo $N_{t}$ definido em um espaço de probabilidade $(\Omega, \Im, P)$ e adaptado para uma filtraçào $\Im_{\ell}$. Fle ć um submartingale e sua taxa de falha acumulada é $A_{t}=\lambda t$, usando a decomposição de Doob-Meyer podemos verificar que $M_{t}=N_{t}-A_{t}$ é um martingale

$$
\begin{aligned}
E\left[M_{t} \mid \Im_{s}\right] & =E\left[N_{t}-A_{t} \mid \Im_{s}\right]=E\left[N_{t}-\lambda t \mid \Im_{s}\right]=E\left[N_{t} \mid \Im_{s}\right]-E\left[\lambda t \mid \Im_{s}\right] \\
& =E\left[N_{t}+N_{s}-N_{s} \mid \Im_{s}\right]-\lambda t=E\left[N_{t}-N_{s} \mid \Im_{s}\right]+E\left[N_{s} \mid \Im_{s}\right]-\lambda t \\
& =E\left[N_{t}-N_{s}\right]+N_{s}-\lambda l-N_{s}-\lambda s=M_{s} .
\end{aligned}
$$

O item 3) da definição de martingale está provado, os itens 1) e 2) seguem de forma trivial. Assim, $A_{t}$ é o compensador do processo de Poisson homogêneo. Como ele é contínuo, do teorema segue que os tempos de salto desse processo são totalmente inacessíveis, isto quer dizer que para cada $T$ instante de salto não existe uma sequência de tempos $\left\{T_{n}\right\}$ tais que $T_{n}<T$ e $\lim _{n} T_{n}=T$.

\subsection{Taxa de Falha do Processo Pontual}

Nesta seção faremos um estudo da taxa de falha $\alpha_{t}$ de um processo pontual $N_{t}$ conforme Aalen (1978). Seja $\left(\Omega, \Im, \Im_{t}, P\right), t \in[0,1]$, o espaço de probabilidade adaptado com a filtração $\Im_{t}$ (contínua à dircita) definidos no início deste capítulo [Seções 2.1 e 2.2]. Assim, vamos assumir que as seguintes suposições são válidlas

1) $E\left[N_{\ell}\right]<\infty, t<\infty$;

2) Os tempos de salto $T_{1}<T_{2}<\ldots$ de $N_{t}$ são totalmete inacessíveis.

Além disso, vamos assumir que existe um processo näo negativo $\alpha$ com trajetórias contínuas à esquerda, com limites à direita $\mathrm{cm}$ cada ponto, adaptado à filtração $\Im_{t} \mathrm{e}$

$$
\Lambda_{t}=\int_{0}^{t} \alpha_{s} d s
$$

O processo $\alpha$ é chamado taxa de falha (ou processo intensidade) de $N$ com respeito à filtração $\Im_{t}$ e a probabilidade $P$.

Teorema 2.4.1. Suponha $N$ é um processo de pontual, e seja $T_{1}, T_{2}, \ldots$ os sucessivos tempos de salto de $N$, isto é, os tempos l lais que $\Delta N_{t}=N_{t}-N_{t-}=1$. Assim,

$$
P\left[T_{n+1}-T_{n} \leq t \mid \Im_{T_{n}}\right] \text { : é absolutamente contínuo em t }
$$


se e somente se o compensador $\Lambda$ de $N$ pode ser escrito como

$$
\Lambda_{t}=\int_{0}^{\iota} \alpha_{s} d s
$$

onde $\alpha_{s}$ é un processo estocástico.

Esse Teorema diz que se a distribuição entre os tempos de falha for absolutamente contínua o compensador do processo será absolutamente contínuo. O seguinte teorema pode scr encontrado em Fleming e Harrington [1991,pg. 131, Teorema 4.2.1] ou Aalen [1978, Lema 3.3, i e ii].

Teorema 2.4.2. Seja $\left\{N_{t}: t \geq 0\right\}$ um processo pontual e $\left\{\Lambda_{t}: t \geq 0\right\}$ o compensador com respeito a uma filtração contínua à direita $\left\{\Im_{t}: t \geq 0\right\}$. Assuma $\Lambda_{t}=\int_{0}^{t} \alpha_{s}$ ds para algum processo a que é contínuo à esquerda, tem limite direita e é limitado por uma variável aleatória integrável $Q$, isto é, $\alpha_{t} \leq Q$, para todo te e $E[Q]<\infty$. Entäo,

(1) $\lim _{h \rightarrow 0} \frac{1}{h} E\left[N_{t+h}-N_{t} \mid \Im_{t}\right]=\alpha_{t+}$

(2) $\lim _{h \rightarrow 0} \frac{1}{h} P\left[N_{t+h}-N_{t}=1 \mid \Im_{t}\right]=\alpha_{t+}$

Prova para o ilem (1)

$$
\begin{aligned}
\lim _{h \rightarrow 0} \frac{1}{h} E\left[N_{t+h}-N_{\ell} \mid \Im_{t}\right]= & \lim _{h \rightarrow 0} \frac{1}{h} E\left[M_{t+h}-M_{t}+A_{t+h}-A_{t} \mid \Im_{t}\right]= \\
& \lim _{h \rightarrow 0} \frac{1}{h}\left\{E\left[M_{t+h} \mid \Im_{t}\right]-E\left[M_{t} \mid \Im_{t}\right]+E\left[\Lambda_{t+h}-\Lambda_{t} \mid \Im_{t}\right]\right\}= \\
& \lim _{h \rightarrow 0} \frac{1}{h} E\left[\Lambda_{t+h}-\Lambda_{t} \mid \Im_{t}\right]=\lim _{h \rightarrow 0} \frac{1}{h} E\left[\int_{t}^{t+h} \alpha_{s} d s \mid \Im_{t}\right]= \\
& E\left[\lim _{h \rightarrow 0} \frac{1}{h} \int_{t}^{t+h} \alpha_{s} d s \mid \Im_{t}\right]=E\left[\alpha_{t+} \mid \Im_{t}\right]=\alpha_{t+}
\end{aligned}
$$

vamos provar agora o item (2), fixemos t e seja $S>t$ o instante do primeiro salto depois de $t$. Desde que $S$ é um tempo de parada, segue que $I_{[\iota, S]}(u)$ é uma família de variáveis aletórias adaptada para $\Im_{u}$, desde que ele é contínuo à esquerda, ele é um processo previsivel. Para $h>0$, definimos a integral estocástica

$$
\int_{t}^{t+h} I_{(t, S\}}(u) d M(u)
$$


este processo, com h variando no tempo é um martingale, assim

$$
\begin{aligned}
\lim _{h \rightarrow 0} \frac{1}{h} P\left[N_{t+h}-N_{t} \geq 1 \mid \Im_{t}\right] & =\lim _{h \downarrow 0} \frac{1}{h} E\left[\int_{t}^{t+h} I_{(t, S]}(u) d N(u) \mid \Im_{t}\right]= \\
& =\lim _{h \downarrow 0} \frac{1}{h} E\left[\int_{t}^{t+h} I_{(t, S\}}(u) d \Lambda(u+) \mid \Im_{t}\right]= \\
& =E\left[\lim _{h \downarrow 0} \frac{1}{h} \int_{t}^{t+h} I_{(t, S]}(u) d \Lambda(u+) \mid \Im_{t}\right]= \\
& =E\left[\alpha(u+) \mid \Im_{t}\right]=\alpha(u+)
\end{aligned}
$$

Agora só precisamos mostrar que

$$
\lim _{h \downarrow 0} \frac{1}{h} P\left[N_{t+h}-N_{t} \geq 2 \mid \Im_{t}\right]=0
$$

Considere agora $S_{1}$ o instante do segundo salto do processo $\mathrm{N}$ depois de t.

$$
\begin{aligned}
\operatorname{limn}_{h \downarrow 0} \frac{1}{h} P\left[N_{t+h}-N_{t} \geq 2 \mid \Im_{t}\right] & =\lim _{h \downarrow 0} \frac{1}{h} E\left[\int_{t}^{t+h} I_{\left(S, S_{1}\right]}(u) d N(u) \mid \Im_{t}\right]= \\
& =\lim _{h \downarrow 0} \frac{1}{h} E\left[\int_{t}^{t+h} I_{(t, S]}(u) d \Lambda(u+) \mid \Im_{t}\right]= \\
& =E\left[\lim _{h \downarrow 0} \frac{1}{h} \int_{l}^{t+h} I_{(t, s]}(u) d \Lambda(u+) \mid \Im_{t}\right]=0
\end{aligned}
$$

c portanto o itern (2) está demonstrado.

No Capítulo 4 vamos estimar a taxa de falha acumulada $A$ do processo pontual $N_{t}$ de forma Bayesiana não-paramétrica para o modelo de intensidade multiplicativa |Aalen (1978)], nesse caso a taxa de falha $\alpha$ tem a seguinte forma

$$
\alpha_{s}=Y_{s} d A_{s}
$$

onde $Y$ é um processo observável da amostra e $A$ é uma função determinística desconhecida. Também vamos apresentar um estimador para a taxa de falha desse processo.

\subsection{Representação do Processo Pontual}

Nesta seção nós apresentamos os tcoremas de existência e unicidade do compensador, esses teoremas garantem que?

Teorema 2.5.1. Seja $F_{1}(t)=P\left(T_{1} \leq t\right)$, e seja

$$
F_{i}(t)=P\left(T_{i} \leq t \mid T_{i-1}, \ldots, T_{1}\right), \quad i \geq 2,
$$


funções distribuiçôes condicionais regulares. Então o compensador $A=\left\{A_{t}, \Im_{t}\right\}, t<+\infty$, do processo pontual $N=\left\{N_{t}, \Im_{t}\right\}, t \geq 0$, pode ser definido pela fórmula

$$
A=\sum_{i \geq 1} A_{i}^{(i)}
$$

onde

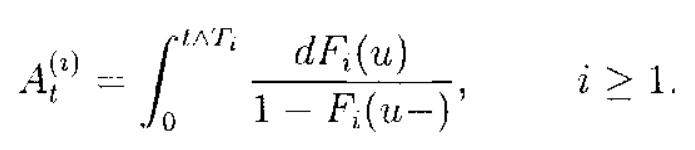

(A) Para fixados $s_{i-1}, \ldots, s_{1}, s_{0}$, a funçäo $Q_{i}\left(s_{i}: s_{i}, \ldots, s_{1}, s_{0}\right)$ é não decrescente, contínua à direita, com saltos que não excedem uma unidade e, para $s_{i-1}<\infty$,

$$
\lim _{s_{i} \downarrow s_{i-l}} Q_{i}\left(s_{i}: s_{i-1}, \ldots, s_{1}, s_{0}\right)=0
$$

(B) $Q_{2}\left(s_{i}: s_{i-1}, \ldots, s_{1}, s_{0}\right)=0$ fora do domínio

$$
\left\{\left(s_{i}, s_{2-1}, \ldots, s_{1}, s_{0}\right): s_{i}>s_{i-1}>\ldots>s_{1}>s_{0}\right\}
$$

(C) $A_{t}^{i}(x)=Q_{i}\left(t \wedge T_{i}(x): T_{i-1}(x), \ldots, T_{1}(x),(0)\right.$.

Teorema 2.5.2 (Teorema de Existência). Seja $Q_{i}\left(s_{i}: s_{i-1}, \ldots, s_{1}, s_{0}\right), i \geq 1$, uma sequência de funçôes Borel satisfazendo $(A)$ e $(B)$. Entäo, no expaço mensurável $(\Omega, \Im)$ existe uma probabilidade $\mu$ lais que o processo

$$
A=\left(A_{t}(x), \Im_{t}, \mu\right), t \geq 0 \quad \text { com } \quad A_{t}(x)=\sum_{i \geq 1} A_{t}^{i}(x)
$$

$$
A_{t}^{i}(x)=Q_{\imath}\left(t \wedge T_{i}(x): T_{i .1}(x), \ldots, T_{1}(x), 0\right),
$$

é um compensador do processo pontual $N=\left(N_{t}, \Im_{t}, \mu\right), t \geq 0$

Teorema 2.5.3 (Teorema de Unicidade). Seja $A=\left(A_{t}(x), \Im_{t}, \mu\right)$ e $B=\left(B_{t}(x), \Im_{t}, \mu\right)$, $t \geq 0$, os compensadores dos processos pontuais $\left(N_{t}(x), \Im_{t}, \mu\right)$ e $\left(N_{t}(x), \Im_{t}, \nu\right), t \geq 0$, respectivamente. Seja $\theta=\theta(x)$ um tempo de parada (com respeito ao sistema $\left(\Im_{t}\right), t \geq 0$ ) tais que, $\mu$ ( ou $\nu)\left(P\right.$-q.c.), $A_{t \wedge \theta}=B_{t \wedge \theta}$. Então as restriçöes $\mu / \Im_{\theta}$ e $\nu / \Im_{\theta}$ das medidas $\mu$ e v para a $\sigma$-álgebra $\Im_{\theta}=\sigma\left(\bigcup_{n} \Im_{\theta \wedge T_{n}}\right)$ coincidem. 


\section{Capítulo 3}

\section{Processos de Lévy Puro Salto}

\subsection{Introdução}

Neste trabalho estaremos assumindo que a taxa de falha acumulada $A$ é um processo Lévy puro salto, chamado de processo Beta. Processos de Lévy têm surgido em vários trabalhos de análise bayesiana não paranétrica, Doksum (1974), Ferguson (1974), Ferguson e Phardia (1979), Ijort (1990), Kim (1999), Kim e Lee (2001) e aparentemente um dos primciros trabalhos sobre o assunto foi de Ferguson e Klass (1972). A importância dessa classe de processos cstá no fato de serem conjugadas, resultado devido a Doksum (1974) e Ferguson e Phadia (1979). Interesse em fazer simulações têm feito alguns autores estudarem a representação desses processos, Damicn, Laud e Smith (1995), Walker e Damien (1998,2000), Wolpert e Ickstad (1998). Aqui, vamos estudar os processos de Lévy puro salto via o compensador.

\subsection{Processos de Lévy Puro Salto}

Para a obtenção de alguns resultados nessa seção precisamos que a base estocástica $\left(\Omega, \Im, \Im_{t}, P\right)_{t \geq 0}$ seja completa. Para completar uma dada base estocástica $\left(\Omega, \Im, \Im_{t}, P\right)_{t \geq 0}$ procedemos conforme Jacod e Shiryaev ¿(1987), pg. 2]. Neste sentido, completamos primeiro a $\sigma$-álgebra $\Im$, denotando por $\mathcal{N}^{P}$ a classe de todos os subconjuntos dos conjuntos de probabilidade nula (com respeito à $P$ ) e por $\Im^{P}$ a menor $\sigma$-álgebra gerada por $\Im \cup \mathcal{N}^{P}$. Da mesma forma, tomamos a menor $\sigma$-álgebra gerada por $\Im_{t}$ e $\mathcal{N}^{P}$ e denotamos por $\Im_{t}^{P}$. Assim, $\left(\Omega, \Im^{P}, \Im_{t}^{P}, P\right)_{t \geq 0}$ é a dada base estocástica completada e, alćm disso, a filtração definida por $\Im_{t}^{P}$ năo perde a propriedade de continuidade à direita 
[Protter (1990), Teorema 31, pg. 22]. Para evitar carregar a notação vamos considerar que $\left(\Omega, \Im, \Im_{t}, P\right)_{t \geq 0}$ é uma base estocástica completa.

Definição 3.2.1 (Processo de Lévy puro salto). Um Processo de Lévy puro salto é um processo estocástico A definido sobre $\left(\Omega, \Im, \Im_{t}, P\right)_{t \geq 0}$, satisfazendo:

a) A é càdlàg e adaptado à filtração $\Im_{t}, t \geq 0$,

b) Para todot $\in[0,1]$ ew $\omega \Omega$

$$
A_{t}(\omega)=\sum_{s} \Delta A_{s}(\omega) I_{\left\{\Delta A_{s}(\omega)>0\right\}}(\omega) I_{\{[0, t]\}}(s)
$$

c) A tem incrementos independentes;

d) $A_{0}(\omega)=0 ; \Delta A_{t}(\omega) \leq 1 ; \forall \omega \in \Omega ; \forall t \in[0,1]$.

Desde que $A$ tem trajetórias càdlàg, ele tem no máximo um número finito de saltos de tamanho maior que $\varepsilon(\varepsilon>0$, fixado) no intervalo $(0,1]$ [Billingsley $(1968)$, Lema 1 , pg. 110]. Logo, $A$ terá no máximo um número enumerável de saltos. Assim, a representação (b) está bem definida. Observe que podemos caracterizar o processo de Lévy puro salto através das distribuições dos saltos e do tamanho do salto. Para isto, associamos uma medida aleatória $\mu$, na forma

$$
\mu(\omega ;[0, t] \times B)=\sum_{s} I_{\left\{\Delta A_{s}(\omega)>0\right\}}(\omega) I_{\{0, t]\}}(s) I_{\{B\}}\left(\Delta A_{s}(\omega)\right)
$$

para $\omega \in \Omega, t \in[0,1]$ e $B \in \beta((0,1])$

Para todo $B \in \beta((0,1])$, definimos tempos de parada com respeito à filtraçăo $\Im_{t}[\mathrm{vcr}$, Delacherie e Meyer (1978), Teorema 44, pg. 66] da seguinte forma

$$
\begin{aligned}
T_{0}^{B}(\omega) & =0 ; \omega \in \Omega \\
T_{1}^{B}(\omega)= & \inf \left\{t>T_{0}^{B}(\omega): I_{\left\{\Delta A_{t}(\omega)>0\right\}}(\omega) I_{\{B\}}\left(\Delta A_{t}(\omega)\right)=1\right\} \\
& \cdot \\
& \cdot \\
T_{n}^{B}(\omega) & =\inf \left\{t>T_{n-1}^{B}(\omega): I_{\left\{\Delta A_{t}(\omega)>0\right\}}(\omega) I_{\{B\}}\left(\Delta A_{t}(\omega)\right)-1\right\}
\end{aligned}
$$


A partir da sequência de tempos de parada $\left\{T_{n}^{B}\right\}$, podemos interpretar $\mu$ como um processo pontual da seguinte forma

$$
\begin{aligned}
\mu(\omega ;\{0, t\} \times B) & =\sum_{s} I_{\left\{\Delta A_{s}(\omega)>0\right\}}(\omega) I_{\{[0, t\}\}}(s) I_{\{B\}}\left(\Delta A_{s}(\omega)\right) \\
& =\sum_{i=1}^{\infty} I_{\left\{T_{i}^{B}<t\right\}}(\omega) .
\end{aligned}
$$

Lema 3.2.1. Para todo $B \in \beta((0,1])$, tomamos uma medida sobre $([0,1] \times(0,1] ; \beta([0,1] \times$ $(0,1]))$, na forma

$$
\nu([0, t] \times B)=E[\mu(\cdot ; 0, t] \times B)]
$$

Além disso, o processo estocástico

$$
M_{t}^{B}(\omega)=\mu(\omega ;[0, t] \times B)-\nu([0, t] \times B)
$$

é um martingale com respeito a filtração $\left\{\Im_{l}\right\}$ e $\nu$ é única, no sentido de que se existe $\widetilde{\nu}$, uma medida sobre $([0,1\} \times(0,1] ; \beta([0,1] \times(0,1]))$ salisfazendo 3.2 , então $\widetilde{\nu}=\nu$.

Prova: Vamos mostrar que de fato $\nu$ é unna medida sobre $([0,1] \times[0,1] ; \beta([0,1] \times$ $(0,1]))$. Assim, devemos mostrar que $u$ satisfaz:

i) $\nu(\emptyset)=0$;

ii) $\nu\left(\bigcup_{i=1}^{\infty}\left(A_{i} \times B_{i}\right)\right)-\sum_{i=1}^{\infty} \nu\left(A_{i} \times B_{i}\right), A_{i} \in \beta([0,1]), B_{i} \in \beta((0,1])$, $\left(A_{i} \times B_{i}\right) \cap\left(A_{j} \times B_{j}\right)=\emptyset, i \neq j$.

O item $i)$ sai direto do fato de $\mu$ ser medida. Considere $\bigcup_{i=1}^{\infty}\left(A_{i} \times B_{i}\right) \operatorname{com}\left(A_{i} \times B_{i}\right) \cap\left(A_{j} \times\right.$ $\left.B_{j}\right)=\emptyset, i \neq j$ c $A_{i} \in \beta([0,1])$ e $B_{i} \in \beta((0,1\})$. Utilizando o Teorema da Convergência Monótona, temos

$$
\begin{aligned}
\nu\left(\bigcup_{i-1}^{\infty}\left(A_{i} \times B_{i}\right)\right) & =E\left[\mu\left(., \bigcup_{i=1}^{\infty}\left(A_{i} \times B_{i}\right)\right)\right]=E\left[\sum_{i=1}^{\infty} \mu\left(., A_{i} \times B_{i}\right)\right] \\
& =E\left[\lim _{n-+\infty} \sum_{i=1}^{n} \mu\left(., A_{i} \times B_{i}\right)\right]=\lim _{n \rightarrow \infty} E\left[\sum_{i=1}^{n} \mu\left(., A_{i} \times B_{i}\right)\right] \\
& =\lim _{n \rightarrow \infty} \sum_{i=1}^{n} E\left[\mu\left(., A_{i} \times B_{i}\right)\right]-\sum_{i=1}^{\infty} E\left[\mu\left(., A_{i} \times B_{i}\right)\right]=\sum_{i=1}^{\infty} \nu\left(A_{i} \times B_{i}\right) .
\end{aligned}
$$


Agora, vamos mostrar que $M_{t}^{B}(\omega)$ é um martingalc. Assim, para $s \leq t$

$$
\begin{aligned}
E\left[M_{t}^{B} \mid \Im_{s}\right]= & E\left[\mu(. ;[0, t] \times B)-\nu([0, t] \times B) \mid \Im_{s}\right] \\
= & E\left[\mu(. ;[0, s] \times B)-\nu([0, s] \times B)+\mu(. ;(s, t] \times B)-\nu((s, t] \times B) \mid \Im_{s}\right] \\
& \text { incrementos independentes } \\
= & \mu(. ;[0, s] \times B)-\nu([0, s] \times B)+E[\mu(. ;(s, t] \times B)-\nu((s, t] \times B)] \\
= & \mu(. ;[0, s] \times B)-\nu([0, s] \times B)-M_{s}^{B}
\end{aligned}
$$

$\Lambda$ unicidade da decomposição de Doob-Meyer [Teorema 2.3.1, capítulo 2] garante que $\nu$ é o compensador do processo pontual $\mu$. Além disso, concluímos que $\mu$ é uma medida aleatória de Poisson no sentido de Jacod e Shiryaev[1987,pag. 70], pois o compensador é determinístico. A medida $\nu$ é única, no sentido que, se uma outra medida $\widetilde{\nu}$ sobre $[0,1] \times(0,1]$ satisfaz as condições do Lema então $\nu=\tilde{\nu}$, de acordo com a decomposição de Doob-Meyer.

Lema 3.2.2. Seja $f:(0,1] \rightarrow \mathbb{R}$ mensurável e integrável (em relaçäo a $\mu$ ). Entäo, para todo $B \in \beta(\{0,1])$

$$
\int_{B} f(x) \mu(\omega ;[0, t] \times d x)=\sum_{s} f\left(\Delta A_{s}(\omega)\right) I_{\left\{\Delta A_{s}\{\omega)>0\right\}}(\omega) I_{\{B\}}\left(\Delta A_{s}(\omega)\right) I_{\{[0, t]\}}(s)
$$

Prova: Segue do fato que $\mu$ é uma medida de contagem.

Assim, se tomarmos $B=(0,1]$ e $f(x)=x$ para todo $x$, concluímos que

$$
\int_{0}^{1} x \mu(\omega ;[0, t] \times d x)-A_{t}(\omega)
$$

para todo $t \in[0,1]$ e $\omega \in \Omega$. Com isso, obtemos que o processo de: Lévy puro salto pode ser caracterizado pela medida alcatória $\mu$.

O seguinte teorema fornece fórmulas que nos permite calcular a esperança e a variância do processo de Lévy $A$.

Teorema 3.2.1. Seja $f:[0,1] \rightarrow \mathbb{R}$ mensurável e integrável (em relação a $\mu$ ) e $\nu$ o compensador do processo pontual $\mu$ associado ao processo de Lévy puro salto A. Entäo, para todo $B \in \beta((0,1])$

$$
\begin{gathered}
E\left[\int_{B} f(x) \mu(. ;[0, t] \times d x) !=\int_{B} f(x) \nu([0, t] \times d x)\right. \\
E\left[\int_{B} f(x) \mu(. ;[0, t] \times d x)-\int_{B} f(x) \nu([0, t] \times d x)\right]^{2}=
\end{gathered}
$$




$$
=\int_{B} f^{2}(x) \nu\left([0, \ell \times d x) \cdots \operatorname{Var}\left[\int_{B} f(x) \mu(\cdot ;[0, t] \times d x)\right]\right.
$$

Prova: Vamos provar 3.4, para isso, vamos primeiro considerar $f(x)$ como sendo uma função simples $f(x)=\sum_{j=1}^{n} a_{j} I_{B_{j}}(x)$. Assim,

$$
\begin{aligned}
E\left[\int_{B} f_{n}(x) \mu(. ;[0, t] \times d x)\right] & =E\left[\int_{B} \sum_{j=1}^{n} a_{j} I_{B},(x) \mu(. ;[0, t] \times d x)\right] \\
& =E\left[\sum_{j=1}^{n} a_{j} \int_{B \cap B_{j}} \mu(. ;[0, t] \times d x)\right] \\
& =E\left[\sum_{j=1}^{n} a_{j} \mu\left(. ;[0, t] \times B \cap B_{j}\right)\right] \\
& =-\sum_{j=1}^{n} a_{j} \nu\left([0, t] \times B \cap B_{j}\right) \\
& =\int_{B} f(x) \nu([0, t] \times d x) .
\end{aligned}
$$

Vamos supor agora que $f(x)$ é uma função mensurável não-negativa. Seja $f_{n}$ uma sequência de funçóes simples, tais que $f_{n}(x) \rightarrow f(x)$ e $f_{n}(x)=\sum_{j-1}^{n} a_{j}^{n} I_{B_{j}^{n}}(x)$. Então, aplicando o Teorema 6.2.1 e o Teorema da Convergência Monótona, temos

$$
\begin{aligned}
E\left[\int_{B} f(x) \mu(. ;[0, t] \times d x)\right] & =E\left[\int_{B} \lim _{n \rightarrow \infty} f_{n}(x) \mu(. ;[0, t] \times d x)\right] \\
& =E\left[\lim _{n} \int_{B} \sum_{j=1}^{n} a_{j}^{n} I_{B_{j}^{n}}(x) \mu(. ;[0, t] \times d x)\right] \\
& =E\left[\lim _{n \rightarrow \infty} \sum_{j=1}^{n} a_{j}^{n} \mu\left(. ;[0, t] \times B \cap B_{j}^{n}\right)\right] \\
& \therefore \lim _{n \rightarrow \infty} E\left[\sum_{j=1}^{n} a_{j}^{n} \mu\left(. ;[0, t] \times B \cap B_{j}^{n}\right)\right] \\
& =\lim _{n \rightarrow \infty} \int_{B} \int_{n}(x) \nu([0, t] \times d x) \\
& =\int_{B} \lim _{n \rightarrow \infty} f_{n}(x) \nu([0, t] \times d x) \\
& =\int_{B} f(x) \nu([0, t] \times d x)
\end{aligned}
$$

Considere $f(x)$ uma função mensurável geral. Temos que toda função mensurável é decomposta como a diferença entre suas partes positiva e negativa, isto é $f=f^{+}-f^{-}$, onde $f^{\prime}$ e $f^{\prime \prime}$ săo funçōes mensuráveis näo-negativas. Dessa forma, aplicando o resultado 


$$
\begin{aligned}
& \text { anterior } \\
& E\left[\int_{B} f(x) \mu(. ;[0, t] \times d x)\right]=E\left[\int_{B}\left[f^{+}(x)-f(x)\right] \mu(. ;[0, t] \times d x)\right] \\
& =E\left[\int_{B} f^{+}(x) \mu(.:[0, t] \times d x)-\int_{I B} f^{-}(x) \mu(\omega ;[0, t] \times d x)\right] \\
& =E\left[\int_{B} f^{+}(x) \mu(. ;[0, t] \times d x)\right]-E\left[\int_{B} f^{-}(x) \mu(. ;[0, t] \times d x)\right] \\
& =\int_{B} f^{+}(x) \nu([0, t] \times d x)-\int_{B} f^{--}(x) \nu([0, t] \times d x) \\
& -\int_{B}\left[f^{+}(x)-f^{-}(x)\right] \nu([0, t] \times d x) \\
& \left.=\int_{B} f(x) \nu(0, t] \times d x\right) .
\end{aligned}
$$

Assim, a prova de 3.4 está terminada. Resta-nos provar 3.5. Seja,

$$
M_{t}^{i}=\mu\left(\omega ;[0, t] \times B_{i}\right)-E\left[\mu\left(. ;[0, t] \times B_{i}\right)\right]
$$

Os $M_{t}^{i}$ são $L^{p}$-martingales, para todo $p \geq 0$ e $L\left[M_{t}^{i}\right]=0$. Supondo que $B_{i}$ e $B_{j}$ são disjuntos. Nós temos

$$
E\left[M_{t}^{i} M_{t}^{j}\right]=E\left[\sum_{k}\left(M_{t_{k+1}}^{i} \cdots M_{t_{k}}^{i}\right) \sum_{l}\left(M_{t_{t+1}}^{j}-M_{t_{i}}^{j}\right)\right]
$$

para qualquer partição $0=t_{0}<t_{1}<\ldots<t_{n}=t$. Usando a propriedade inartingale, temos

$$
E\left[M_{t}^{i} M_{t}^{j}\right]=E\left[\sum_{k}\left(M_{i_{k+1}}^{i}-M_{i_{k}}^{i}\right)\left(M_{t_{k+1}}^{j}-M_{t_{k}}^{j}\right)\right]
$$

e da desigualdade $|a b| \leq\left(a^{2}+b^{2}\right)$

$$
\sum_{k}\left(M_{l_{k+1}}^{i}-M_{t_{k}}^{i}\right)\left(M_{t_{k+1}}^{j}-M_{t_{k}}^{j}\right) \leq\left(\sum_{k}\left(M_{t_{k+1}}^{i}-M_{t_{k}}^{i}\right)\right)^{2}+\left(\sum_{k}\left(M_{t_{k+1}}^{j}-M_{t_{k}}^{j}\right)\right)^{2}
$$

Além disso

$$
\left.\left(\sum_{k}\left(M_{t_{k+1}}^{i}-M_{t_{k}}^{i}\right)\right)^{2} \leq\left(\mu\left(\omega ;[0, t] \times B_{i}\right)\right)^{2}+\left(E\left[\mu(\because ; 0, t] \times B_{i}\right)\right]\right)^{2} .
$$

Portanto as somas são dominadas por uma variável aleatória integrável. Desde que $M_{t}^{i}$ e $M_{t}^{j}$ tem trajetórias de variação finita em $[0, t]$, nós podemos tomar uma sequência $\left(\pi_{n}\right)_{n \geq 1}$ de partições onde o $\max _{k}\left|t_{k}-t_{k-1}\right| \longrightarrow 0$, nós temos

$$
\left.E\left[M_{t}^{i} M_{t}^{j}\right]=E\left[\sum_{0<s \leq t} \Delta M_{s}^{i} \Delta M_{s}^{j}\right]=E\left[\sum_{0<s<t} \Delta \mu(; ; 0, s] \times B_{i}\right) \Delta \mu\left(; ;[0, s] \times B_{j}\right)\right]=0
$$


$A$ esperança acima é 0 porque, se $\Delta \mu\left(\omega ;[0, s] \times B_{i}\right)=1$ no instante $s$ então, $\Delta A_{s} \in B_{i}$, (aso contrário é 0 e como $B_{i}$ e $B_{j}$ são disjuntos, $\Delta A_{s}$ não pode saltar no mesmo instante para lugares distintos. Considere agora $f(x)=\sum_{j=1}^{n} a_{j} I_{B_{j}}(x)$ uma função simples, temos

$$
\begin{aligned}
& E\left[\int_{B} f(x) \mu(. ;[0, t] \times d x) \cdots \int_{B} f(x) \nu([0, t] \times d x)\right]^{2} \\
= & E\left[\int_{B} \sum_{j-1}^{n} a_{j} I_{B_{j}}(x) \mu(. ;[0, t] \times d x)-\int_{B} \sum_{j=1}^{n} a_{j} I_{B_{j}}(x) \nu([0, t] \times d x)\right]^{2} \\
= & E\left[\sum_{j=1}^{n} a_{j} \mu\left(. ;[0, t] \times B \cap B_{j}\right)-\sum_{j=1}^{n} a_{j} \nu\left([0, t] \times B \cap B_{j}\right)\right]^{2} \\
= & E\left[\sum_{j=1}^{n} a_{j}\left[\mu\left(. ;[0, t] \times B \cap B_{j}\right)-\nu\left([0, t] \times B \cap B_{j}\right)\right]\right]^{2} \\
= & E\left[\sum_{j=1}^{n} a_{j} M_{t}^{j}\right]^{2}=\sum_{j=1}^{n} a_{j}^{2} E_{i}\left[M_{t}^{j}\right]^{2}+\sum_{r=1}^{n} \sum_{s=1}^{n} a_{r} a_{s} E\left[M_{t}^{\tau} M_{t}^{s}\right] \\
= & \sum_{j=1}^{n} a_{j}^{2} \nu\left([0, t] \times B \cap B_{j}\right)-\int_{B} \sum_{j=1}^{n} a_{j}^{2} I_{B_{j}}(x) \nu([0, t] \times d x) \\
= & \int_{B} f^{2}(x) \nu([0, t] \times d x) .
\end{aligned}
$$

O resto da prova segue da mesma forma como foi feito para 3.4.

Considere o processo,

$$
\begin{aligned}
H_{t}(\omega) & =A_{t}(\omega)-E\left[A_{t}\right]- \\
& =A_{t}(\omega)-\int_{0}^{1} x \nu([0, t] \times d x)
\end{aligned}
$$

pois,

$$
E\left[A_{t}\right]=L i\left[\int_{0}^{1} x \mu(\cdot ;[0, t] \times d x)\right]=\int_{0}^{1} x \nu\left(\left[0, t_{1} \times d x\right)\right.
$$

Proposiçāo 3.2.1. O processso $H_{t}$ é um martingale com respeito à filtraçâo $\left\{\Im_{t}\right\}$.

Prova: É semelhante à prova feita no lema 3.2.1.

Desde que $A$ é um submartingale, obtemos da decomposição de Doob-Meyer que $E\left[A_{t}\right]$ é o compensador do processo de Lévy puro-salto $A_{t}$. 


\subsection{Representação do Processo de Lévy}

Considere $A: \Omega \times(0,1] \rightarrow[0,1]$ um processo de Lévy puro salto. Então, $A$ tém a seguinte representação

$$
A_{t}(\omega)=\sum_{s} \Delta A_{s}(\omega) I_{\left\{A_{s}(\omega)>0\right\}}(\omega) I_{\{\lfloor[0, t\}\}}(s), t \in[0,1], \omega \in \Omega .
$$

Nesta seção, vamos introduzir uma representação para o processo de Lévy que será utilizada para obtermos um algoritmo de simulação de tais processos. Nossa representação é uma gencralização da representação de Ferguson c Klass(1972) : Walker e Damicn (2000).

Seja $B_{k}^{(n)}=\left(\frac{k}{2^{n}}, \frac{k+1}{2^{n}}\right\rfloor$ um intervalo diádico para $k=0,1, \ldots, 2^{n}-1$ e $n \in \mathbb{N}$. Assim, utilizando a Equação 3.1, temos que

$$
\mu\left(\omega,[0, t] \times B_{k}^{(n)}\right)=\sum_{i=1}^{\infty} I_{\left\{T_{i}^{b_{k}^{(n)}}(\omega) \leq t\right\}}(\omega)
$$

Ao denotarmos

$$
S_{i}^{(n)}(\omega)=\sum_{k=0}^{2^{n}-1} T_{i}^{\beta_{k}^{(n)}}(\omega)
$$

obtemos que $S_{i}^{(n)}$ é um tempo de parada, e

$$
\begin{aligned}
& \sum_{k=0}^{2^{n}-1} \mu\left(\omega,[0, t] \times B_{k}^{(n)}\right)=\sum_{k=0}^{2^{n} \cdots 1} \sum_{i=1}^{\infty} I_{\left\{T_{i}^{B_{k}^{(n)}}(\omega)<t\right\}}(\omega)= \\
& \sum_{i=1}^{\infty} \sum_{k=1}^{2^{2 n}-1} I_{\left\{T_{i}^{B_{k}^{(n)}}(\omega) \leq t\right\}}(\omega)=\sum_{i=1}^{\infty} I_{\left\{S_{i}^{(n)}(\omega) \leq t\right\}}(\omega),
\end{aligned}
$$

pois,

$$
\bigcup_{k-0}^{2^{n} \cdots 1}\left\{T_{2}^{R_{k}^{(n)}}(\omega) \leq t\right\}=\left\{S_{i}^{(n)}(\omega) \leq t\right\} .
$$

Assim, concluímos que

$$
\mu(\omega,[0, t] \times(0,1])=\sum_{k=0}^{2^{n}-1} \mu\left(\omega,[0, t] \times B_{k}^{(n)}\right)=\sum_{i=1}^{\infty} I_{\left\{S_{i}^{(n)}(\omega)<t\right\}}(\omega), \forall n \in \mathbb{N} .
$$

Ao tomarmos,

$$
S_{i}(\omega)=\lim _{n \rightarrow \infty} S_{i}^{(n)}(\omega), \forall \omega \in \Omega,
$$

obtemos que $S_{i}$ é um tempo de parada, e

$$
\mu(\omega,[0, t] \times(0,1])=\lim _{n \rightarrow \infty} \sum_{i=1}^{\infty} I_{\left\{s_{i}^{(n)}(\omega) \leq t\right\}}(\omega)=
$$




$$
-\sum_{i=1}^{\infty} \lim _{n \rightarrow \infty} I_{\left\{S_{i}^{(n)}(\omega) \leq t\right\}}(\omega)=\sum_{i-1}^{\infty} I_{\left\{S_{i}(\omega) \leq t\right\}}(\omega)
$$

pois,

$$
\left\{\omega: S_{i}^{(n)}(\omega) \leq t\right\}=\left\{\omega: S_{i}(\omega) \leq t\right\}
$$

Assim, concluímos que a medida aleatória $\mu$ pode ser representada por

$$
\mu(\omega ;[0, t] \times B)=\sum_{i=1}^{\infty} I_{\{B\}}\left(\Delta A_{S_{i}(\omega)}(\omega)\right) I_{\left\{S_{2}(\omega) \leq t\right\}}(\omega)
$$

para todo $\omega \in \Omega, t \in[0,1]$ e $B \in \beta((0,1])$.

Além disso, obtemos que para todo $t \in[0,1]$ e $\omega \in \Omega$,

$$
A_{t}(\omega)=\int_{0}^{1} x \mu(\omega ;[0, t] \times d x)=\sum_{i=1}^{\infty} \Delta A_{S_{i}(\omega)}(\omega) I_{\left\{S_{\imath}(\omega) \leq t\right\}}(\omega)=\int_{0}^{t} \Delta A_{s} d G_{s}(\omega),
$$

onde $G_{t}(\omega)=\sum_{i=1}^{\infty} I_{\left\{S_{i}(\omega)<t\right\}}(\omega)$ corresponde ao processo pontual que conta o número de saltos do processo de Lévy puro salto A. Com isso, obtemos o seguinte lema.

Lema 3.3.1. Considere A um processo de Lévy puro salto, então

$$
A_{t}(\omega)=\sum_{i=1}^{\infty} \Delta A_{S_{i}(\omega)}(\omega) I_{\left\{S_{i}(\omega)<t\right\}}
$$

Na maioria dos processos de Lévy puro salto encontrados na prática, o compensador $\nu$ é dado por

$$
\nu([0, t] \times B)=\int_{0}^{t} \int_{B} f_{s}(x) d x d s
$$

Lema 3.3.2. O compensador do processo de salto $G_{t}$ é dado por:

$$
\nu([0, t] \times(0,1])=\int_{0}^{t}\left[\int_{0}^{1} f_{s}(x) d x\right] d s ; t \in[0,1] .
$$

Prova: Inicialmente, temos que

$$
\nu(\omega ;[0, t] \times B)-\nu([0, t] \times B)
$$

é um martingale. Assim, concluímos que

$$
\sum_{i=1}^{n} I_{\left\{S_{i} \leq t\right\}}-\int_{0}^{t}\left[\int_{0}^{1} f_{s}(x) d x\right] d s
$$

é um martingale com respeito a filtração $\Im_{t}$. Finalmente, obtemos da unicidade da decomposição de Doob-Meyer que o compensador é único.

Observe que o processo pontual $G_{t}$ é um processo de Poisson năo-homogêneo com taxa de lalha

$$
\int_{0}^{1} f_{s}(x) d x
$$




\subsection{Processos Beta}

Vamos fazer uma aplicação aos processos beta [Kim(1999),Hjort(1990)]. Assim, considere $\lambda_{0}, \alpha$ e $\beta$ funções contínuas estritamente positivas definidas em $[0,1]$ e $A_{0}=\int_{0}^{t} \lambda_{0}(s) d s$, para todo $t \in[0,1]$. Vamos denotar por $b(x: a, b)$ a densidade da distribuição beta com parâmetros $a, b>0$, ou scja

$$
b(x: a, b) \div \frac{\Gamma(a+b)}{\Gamma(a) \Gamma(b)} x^{a-1}(1-x)^{b-1}, 0<x<1 .
$$

Assim, o processo beta com parâmetros $\left(A_{0}, \alpha(t), \beta(t)\right)$ é um processo de Lévy $A$ com compensador dado por

$$
\begin{aligned}
\nu([0, t] \times B) & =\int_{0}^{t} \int_{B} \frac{1}{x} b(x: \alpha(s), \beta(s)) d x d A_{0}(s) \\
& =\int_{0}^{t} \int_{B} \frac{1}{x} b(x: \alpha(s), \beta(s)) d x \lambda_{0}(s) d s \\
& =\int_{0}^{t} \int_{B} \frac{1}{x} \frac{\Gamma(\alpha(s)+\beta(s))}{\Gamma(\alpha(s))+\Gamma(\beta(s))} x^{\alpha(s)-1}(1-x)^{\beta(s)-1} d x \lambda_{0}(s) d s .
\end{aligned}
$$

Considerando o 'Teorema 3.2.1, vamos calcular o valor esperado e a varianciat do processo de Lévy $A$

$$
\begin{aligned}
E\left[A_{t}\right] & =\int_{0}^{1} x \nu([0, t] \times d x)=\int_{0}^{1} \int_{0}^{t} b(x: \alpha(s), \beta s) d A_{0}(s) d x \\
& =\int_{0}^{t} \int_{0}^{1} \frac{\Gamma(\alpha(s)+\beta(s))}{\Gamma(\alpha(s)) \Gamma(\beta(s))} x^{\alpha(s)-1}(1-x)^{\beta(s)-1} d x \lambda_{0}(s) d s \\
& =\int_{0}^{t} \lambda_{0}(s) d s
\end{aligned}
$$

$$
\begin{aligned}
\operatorname{Var}\left[A_{t}\right] & =\int_{0}^{1} x^{2} \nu([0, t] \times d x)=\int_{0}^{1} \int_{0}^{t} x b(x: \alpha(s), \beta s) d A_{0}(s) d x \\
& =\int_{0}^{t} \int_{0}^{1} \frac{\Gamma(\alpha(s)+\beta(s))}{\Gamma(\alpha(s)) \Gamma(\beta(s))} x^{\alpha(s)}(1 \cdots x)^{\beta(s)-1} d x \lambda_{0}(s) d s \\
& =\int_{0}^{t} \frac{\alpha(s)}{\alpha(s)+\beta(s)} \lambda_{0}(s) d s .
\end{aligned}
$$

Quando $\alpha(s) \doteq 1$ para todo $s \in[0,1]$ teremos o processo beta de Hjort(1990) com parâmetros 1 e $\beta(s)$. Logo,

$$
\operatorname{Var}\left[A_{t}\right]=\int_{0}^{t} \frac{\lambda_{0}(s)}{1+\beta(s)} d s
$$


Os saltos do processo beta é um processo de Poisson não-homogêneo com taxa de falha

$$
\int_{0}^{1} \frac{1}{x} b(x: \alpha(s), \beta(s)) d x \lambda_{0}(s)=: E\left[\frac{1}{X_{s}}\right] \lambda_{0}(s),
$$

onde $X_{s}$ tem distribuição beta com parâmetros $\alpha(s)$ e $\beta(s)$, para todo $s \in[0,1]$. 


\section{Capítulo 4}

\section{Inferência Bayesiana}

\subsection{Introdução}

Sejam $X_{1}, \ldots, X_{n}$ variáveis alealórias positivas independentes e identicamente distribuídas com função de distribuição $F$. O objetivo consiste em estimar $F$; onde o parâmetro de interesse (F) pertence a um espaço de funçōes. Assim, para aplicar o enfoque Bayesiano, precisamos definir uma probabilidade sobre o espaço das funçōes de distribuição acumulada. Para isto, basta construirmos um processo estocástico cujas trajetórias são funções de distribuição acumulada. Neste sentido, destacamos os processos de Dirichlet [Ferguson $(1973),(1974)]$; processos tailfree e neutral to the right [Doksum (1974)]. Se F é absolutamente contínua com função densidade $f$, Dykstra e Laud (1981) utilizaram processos gama estendido como distribuição à priori para a taxa de falha $\lambda$. Ao invés de estimar a função de distribuição acumulada $F$, Hjort (1990) propôs um método Bayesiano para estimar a taxa de falha acumulada $A$. Se $F$ é absolutamente contínua, então, os resultados obtidos para estimar $F$ podem ser facilmente adaptados para estimar $A$, pois

$$
F^{\prime}(t)=1-\exp \{-A(t)\}, \forall t \in\{0, \infty) .
$$

Além disso, sabemos que $F$ é absolutamente contínua se, e somente se, $A$ também é absolutamente contínıa [Liptser e Shiryayev, Corolário, pg. 249, (1978)]. Por outro lado, todos os processos estoćásticos utilizados para definir a distribuição à priori são processos de Lévy puro salto. Assim, todos os processos estocásticos à priori apresentam trajetórias do tipo escada, consequentemente não são absolutamente contínuas. Desta forma, os resultados apresentados para estimar $F$ não são diretamente adaptados para estimar $A$ e vice-versa. Neste trabalho, vamos apresentar métodos para estimar a taxa de falha 
acumulada $A$, como apresentado em Hjort (1990) c Kim (1999).

Considere a base cstocástica $\left(\Omega, \Im, \Im_{t}, P\right)_{t \geq 0}$ satisfazendo as condições usuais, vamos assumir que o processo pontual $N_{t}$ está definido em $[0,1]$, embora ele possa ser generalizado para o intervalo $[0, \tau]$ e que o processo $\Lambda_{t}$ satisfaz o modelo de intensidade multiplicativo

$$
\Lambda_{t}=\int_{0}^{t} Y_{s} d A(s)
$$

onde $Y$ é um processo previsível (observável) e $A$ é uma função determinística desconhecida, denominada taxa de falha acumulada. Ahaixo, definimos o espaço das taxas de falha acumulada.

$$
\begin{aligned}
\mathcal{A}= & \{\text { classe das funçôes não decrescentes } A \text { contínuas à direita } \\
& \text { definidas em }[0,1], \Delta A \leq 1, A(0)=0\}
\end{aligned}
$$

Para esta classe considere $\Sigma_{\mathcal{A}}$ a $\sigma$-álgebra gerada pelos cilindros. O espaço das observações corresponde ao $\Omega$ definido na seção $2.2 \mathrm{com}$ a $\sigma$-álgebra associada $\Im$.

\subsection{Estimação Bayesiana Não-Paramétrica}

Para aplicarmos o enfoque Bayesiano, tomamos uma probabilidade à priori sobre o espaço $\left(\mathcal{A}, \Sigma_{\mathcal{A}}\right)$ induzida por um processo de Lévy com trajetórias $\mathrm{em} \mathcal{A}$. Na seqüência, utilizamos o modelo de intensidade multiplicativa de Aalen,

$$
\Lambda_{t}=\int_{0}^{t} Y_{s} d A(s)
$$

para construir uma única probabilidade $P^{A}$ sobre $\left(\Omega \times \mathcal{A} ; \Im \times \Sigma_{\mathcal{A}}\right)$ [Teorema 2.5.2], tal que a marginal sobre $\mathcal{A}$ coincide com a probabilidade induzida pelo processo de Lévy $A$. Além disso, dado $A$, o compensador do processo pontual satisfaz o modelo de intensidado multiplicativa.

A distribuição à posteriori de $A$ dado $N$ está bem definida pois $\Omega$ é um espaço de Radon e $\mathcal{A}$ é um espaço métrico completo (com a topologia de Skorohod). Aqui, vamos estudar o processo à posteriori $A_{i} N$. Hjort (1990) mostrou que se o produto integrado

$$
F_{t}-1-\prod_{s \leq t}\left[1-\Delta A_{s}\right]
$$

for finito $(P-q . c)$ e se $A$ é um processo de Lévy puro salto, entāo, o processo à posteriori $A \mid N$ também é um processo de Lévy puro salto. Além disso, Hjort (1990) introduziu a 
classe dos processos beta e mostrou que se $A$ é um processo beta, então, o processo à posteriori também é beta.

Finalmente, Kim (1999) utilizou a caracterização dos processos de Lévy puro salto via o compensador [Capítulo 3] para generalizar os resultados de Hjort (1990). Sem a utilização da hipótese da existencia do produto integrado, Kim (1999) calculou o compensador do processo à posteriori $A \mid N$ dado como priori um processo de Lévy puro salto.

Um processo de Lévy puro salto tem a seguinte representação [ver Definição 3.2.1, Capítulo 3]

$$
A_{t}(\omega)=\sum_{s} \Delta A_{s}(\omega) I_{\left\{\Delta A_{s}(\omega)>0\right\}}(\omega) I_{\{[0, t]\}}(s)
$$

indicando que sua trajetória é uma função escada crescente com $\Delta A_{s}$ o tamanho do salto c $I_{\left\{\Delta A_{s}(\omega)>0\right\}}(\omega) I_{\{[0, t]\}}(s)$ correspondente ao tempo do salto. Definimos uma medida $\mu \mathrm{em}$ $\Omega \times[0,1] \times(0,1]$ como

$$
\mu(\omega ;[0, t] \times B)=\sum_{s} I_{\left\{\Delta A_{s}\{\omega)>0\right\}}(\omega) I_{\{[0, t]\}}(s) I_{\{B\}}\left(\Delta A_{s}(\omega)\right)
$$

para $\omega \in \Omega, t \in[0,1]$ e $B \in \beta((0,1])$. Com isso, obtemos que

$$
\left.A_{t}(\omega)=\int_{0}^{1} x \mu(. ; 0, t] \times d x\right)
$$

c a medida $\mu$ caracteriza o processo de Lévy puro salto. Obtemos de Jacod [1979, pg. 80] que $\mu$ é uma medida aleatória de Poisson. Se definirmos uma medida $\sigma$-finita $\nu$ em $[0,1] \times(0,1]$ por

$$
\nu([0, t] \times B)=E[\mu(\omega,[0, t] \times B)], t \in[0,1], \quad B \in \beta((0,1]),
$$

obtemos do Teorema 3.2.1 que

$$
E\left[A_{t}\right]=\int_{0}^{1} x \nu([0, t] \times d x)-\int_{0}^{1} \int_{0}^{t} x \nu(d s \times d x)
$$

$$
\operatorname{Var}\left[A_{t}\right]=\int_{0}^{1} x^{2} \nu([0, t] \times d x)=\int_{0}^{1} \int_{0}^{t} x^{2} \nu(d s \times d x)
$$

Além disso, obtemos do Teorema 3.1.1 em Jacod (1979, pg. 70) que o compensador $\nu$ caracteriza a medida alcatória $\mu$. Assim, obtemos da Equação 4.1 que o compensador $\nu$ caracteriza o processo de Lévy puro salto.

Vamos supor que o compensador à priori para o processo de Lévy $A$ é dado por

$$
\nu([0, t] \times B)=\int_{0}^{t} \int_{B} d F_{s}(x) d s, \quad t \in[0,1], \quad B \in \beta((0,1])
$$


onde $F_{s}$ são medidas finitas em $[0,1]$ tais que $\int_{0}^{1} y d F_{s}(y)<\infty$, para todo $s \in[0,1]$.

Concluímos do 'Teorema $3.1 \mathrm{em}$ Kim (1999) que, dado as observaçoes do processo pontual o processo à posteriori é também um processo de Lévy co compensador à posteriori é dado por

$$
\begin{aligned}
\nu^{p}([0, t] \times B)= & \int_{0}^{t} \int_{B}(1-x)^{Y_{s}} d F_{s}(x) d s \\
& +\int_{0}^{t} c^{-1}(s) \int_{B} x^{\Delta N_{s}}(1-x)^{Y_{s}-\Delta N_{s}} d F_{s}(x) \frac{1}{\Delta N_{s}} d N_{s}
\end{aligned}
$$

onde $N_{s}=\sum_{i=1}^{n} I_{\left\{T_{i} \leq t\right\}}, \Delta N_{s}=N_{s}-N_{s-}, Y_{s}=\sum_{i=1}^{n} I_{\left\{T_{i} \geq t\right\}}$ c

$$
c(s)=\int_{0}^{1} x^{\Delta N_{s}}(1-x)^{Y_{s} \cdots \Delta N_{s}} d F_{s}(x)
$$

Desta forma o estimador de Bayes de $A$ será dado pela esperança condicional

$$
\begin{aligned}
E\left[A_{t} \mid N\right]= & \int_{0}^{t} \int_{0}^{1} x \nu^{p}(d s, d x)=\int_{0}^{t} \int_{0}^{1} x(1-x)^{Y_{s}} d F_{s}(x) d s \\
& +\int_{0}^{l} c^{-1}(s) \int_{0}^{l} x x^{\Delta N_{s}}(1-x)^{Y_{s}-\Delta N_{s}} d F_{s}(x) \frac{1}{\Delta N_{s}} d N_{s}
\end{aligned}
$$

\subsection{Estimador Bayesiano Não-Paramétrico para a}

\section{Taxa de Falha Acumulada}

Considere $A$ um processo de Lévy com compensador $\nu$ dado por 4.2. Vamos definir funçöes de distribuição $G_{t}$ sobre $[0,1]$ por

$$
G_{t}(x)=\frac{\int_{0}^{x} y d F_{t}(y)}{\lambda_{0}(t)}
$$

onde $\lambda_{0}(t)=\int_{0}^{1} x d F_{t}(x)$. Logo,

$$
d G_{t}(x) \lambda(t)=x d F_{t}(x)
$$

e a esperança e variância ficam, respectivamente,

$$
\begin{gathered}
E\left[A_{t}\right]=\int_{0}^{t} \int_{0}^{1} x \nu(d s \times d x)=\int_{0}^{t} \int_{0}^{1} x d F_{s}(x) d s-\int_{0}^{t} \int_{0}^{1} x d F_{s}(x) d s \\
=\int_{0}^{t} \int_{0}^{1} d G_{s}(x) \lambda_{0}(s) d s=\int_{0}^{t} \lambda_{0}(s) d s . \\
\operatorname{Var}\left[A_{t}\right]=\int_{0}^{t} \int_{0}^{1} x d G_{s}(x) \lambda_{0}(s) d s .
\end{gathered}
$$

e 
Então para escolhermos um processo de Lévy correspondendo à uma informação à priori dado pela média e variância, simplesmente escolhemos uma função $\lambda_{0}(s)$ e uma função $G_{t}(x)$ apropriadias.

Se considerarmos as funções de distribuição $G_{t}(x)$ como sendo distribuições beta com parâmetros $\alpha(t)$ e $\beta(t)$ e denotando por $b(x: a, b)$ a função densidade de probabilidade da distribuição beta com parâmetros $a$ e $b$, obtemos

$$
\begin{aligned}
\nu^{p}([0, t] \times B)= & \int_{0}^{t} \int_{B}(1-x)^{Y_{s}} d F_{s}(x) d s \\
& +\int_{0}^{t} c^{-1}(s) \int_{B} x^{\Delta N_{s}}(1-x)^{Y_{s}-\Delta N_{s}} d F_{s}(x) \frac{1}{\Delta N_{s}} d N_{s} \\
= & \int_{0}^{t} \int_{B}(1-x)^{Y_{s}} \frac{1}{x} d G_{s}(x) \lambda_{0}(s) d s \\
& +\int_{0}^{t} c^{-1}(s) \int_{B} x(1-x)^{Y_{s}-1} \frac{1}{x} d G_{s}(x) \lambda_{0}(s) d N_{s} \\
= & \int_{0}^{t} \int_{B}(1-x)^{Y_{s}} \frac{1}{x} b(x: \alpha(s), \beta(s)) d x \lambda_{0}(s) d s \\
& +\int_{0}^{t} c^{-1}(s) \int_{B} x(1-x)^{Y_{s}-1} \frac{1}{x} b(x: \alpha(s), \beta(s)) d x \lambda_{0}(s) d N_{s} \\
= & \int_{0}^{t} \int_{B}(1-x)^{Y_{s}} \frac{1}{x} b(x: \alpha(s), \beta(s)) d x \lambda_{0}(s) d s \\
& +\int_{0}^{t} \int_{B} b\left(x: \alpha(s), \beta(s)+Y_{s}-1\right) d x d N_{s} .
\end{aligned}
$$

Com isso, o estimador de Bayes é dado por:

$$
\begin{aligned}
\widehat{A}(t)= & E[A(t) \mid N]=\int_{0}^{t} \int_{0}^{1} x \nu^{p}(d s \times d x) \\
= & \int_{0}^{t} \int_{0}^{1}(1-x)^{Y_{s}} b(x: \alpha(s), \beta(s)) d x \lambda_{0}(s) d s \\
& +\int_{0}^{t} \int_{0}^{1} x b\left(x: \alpha(s), \beta(s)+Y_{s}-1\right) d x d N_{s} \\
= & \int_{0}^{t} \frac{\Gamma(\alpha(s)+\beta(s)) \Gamma\left(\alpha(s)+Y_{s}\right)}{\Gamma(\beta(s)) \Gamma\left(\alpha(s)+\beta(s)+Y_{s}\right)} \lambda_{0}(s) d s \\
& +\int_{0}^{t} \frac{\alpha(s)}{\alpha(s)+\beta(s)+Y_{s}-1} d N_{s} .
\end{aligned}
$$

Como exemplo vamos considerar o processo Beta definido por Hjort $(1990)(\alpha(s)=$ $1 \forall s \in[0,1 ;)$, na forma,

$$
\lambda_{0}(t)=\frac{d A_{0}(t)}{d t}, G_{t}(x)=\int_{0}^{x} \beta(t)(1-y)^{\beta(t)-1} d y
$$


onde $G_{t}(x)$ corresponde à distribuição Beta com parâmetros 1 e $\beta(t)$. Ao tomarmos o processo beta como distribuição à priori, obtemos que

$$
\begin{aligned}
\widehat{A}(t) & =E[A(t) \mid N]=\int_{0}^{t} \int_{B} x \nu^{p}(d s \times d x) \\
& =\int_{0}^{t} \frac{\beta(s) \lambda_{0}(s)}{\beta(s)+Y_{s}} d s+\int_{0}^{t} \frac{d N(s)}{\beta(s)+Y_{s}} .
\end{aligned}
$$

Hjort (1990) propóe que um estimador para a taxa de falha, baseado no estimador Bayesiano da taxa de falha acumulada, seja dado por

$$
\hat{\lambda}(s)=\frac{\beta(s) \lambda_{0}(s) d s}{\beta(s)+Y_{s}}+\frac{1}{\beta(s)+Y_{s}}
$$

Propomos que a função $\beta(s)$ seja determinada por $n S(s)$, onde

$$
S(s)=\exp \left\{\cdot\left(\frac{s}{a}\right)^{b}\right\}
$$

é a função de confiabilidade da distribuição Weibull, pois, conforme notado por Hjort [1990, pg. 1264], $\beta(s)$ pode ser interpretada como o conjunto de risco em $s$ à priori. Tomando $v=1 / a^{b}$ temos,

$$
\widehat{A}(t)=\int_{0}^{l} \frac{n \exp \left\{-\widehat{v} s^{\hat{b}}\right\} \widehat{v} \widehat{b} s^{\hat{b}-1}}{n \exp \left\{-\widehat{v} s^{\hat{b}}\right\}+Y(s)} d s+\int_{0}^{t} \frac{d N_{s}}{n \exp \left\{-\widehat{v} s^{\hat{b}}\right\}+Y(s)}
$$

onde $\widehat{a} \hat{b} \mathrm{c} \widehat{v}=1 / \widehat{a}^{\widehat{v}}$ são os estimadores de máxima verossimilhança de $a, b$ e $v$, respectivamente. Logo,

$$
\begin{aligned}
\hat{\lambda}(s) & =\frac{\beta(s) \lambda_{0}(s)}{\beta(s)+Y_{s}}+\frac{1}{\beta(s)+Y_{s}^{\prime}} \\
& =\frac{n \exp \left\{-\widehat{v} s^{\hat{b}}\right\} \widehat{v} \widehat{b} s^{\hat{b}-1}}{n \exp \left\{-\widehat{v} s^{\hat{b}}\right\}+Y_{s}}+\frac{1}{n \exp \left\{-\widehat{v} s^{\hat{v}}\right\}+\overline{Y_{s}}} .
\end{aligned}
$$

As estimativas de máxima verossimilhança para os parâmetros $a$ e $b$ apresentados na seção 1.1 .3 são, respectivamnnte, $\widehat{a}=462.13$ e $\widehat{b}=1.6099 \operatorname{com} \widehat{v} \simeq 5.128 \times 10^{-55}$. 
A variância do processo posteriori é dada por

$$
\begin{aligned}
\operatorname{Var}\left(\hat{A}_{t}\right)= & \int_{0}^{t} \int_{0}^{t} x^{2}-\sum_{s \leq t}\left(\int_{0}^{1} x \nu^{\nu}(\{x\}, d x)\right)^{2} \\
= & \int_{0}^{t} \int_{0}^{1} x(1 \cdots x)^{Y_{s}} b(x: \alpha(s), \beta(s)) d x d A_{0}(s) \\
& +\int_{0}^{t} \int_{0}^{1} x^{2} b\left(x: \alpha(s), \beta(s)+Y_{s}-1\right) d x d N_{s} \\
& -\int_{0}^{t}\left(\int_{0}^{l} x b\left(x: \alpha(s), \beta(s)+Y_{s}-1\right) d x\right)^{2} d N_{s} \\
= & \int_{0}^{t} \alpha(s) \frac{\Gamma(\alpha(s)+\beta(s)) \Gamma\left(\beta(s)+Y_{s}\right)}{\Gamma^{2}(\beta(s)) \Gamma\left(\alpha(s)+\beta(s)+Y_{s}+1\right)} d A_{0}(s) \\
& +\int_{0}^{t} \frac{\alpha(s)\left(\beta(s)+Y_{s}-1\right)}{\left(\alpha(s)+\beta(s)+Y_{s}-1\right)^{2}\left(\alpha(s)+\beta(s)+Y_{s}\right)} d N_{s} .
\end{aligned}
$$

Considerando novamente o processo beta de Hjort(1990) com $\alpha(s)=1$, obtemos

$$
\begin{aligned}
\operatorname{Var}\left(\widehat{A}_{t}\right)= & \int_{0}^{t} \frac{\beta(s)}{\left(\beta(s)+Y_{s}+1\right)\left(\beta(s)+Y_{s}\right)} \lambda_{0}(s) d s \\
& +\int_{0}^{t} \frac{\left(\beta(s)+Y_{s}-1\right)}{\left.\beta(s)+Y_{s}\right)^{2}\left(\beta(s)+Y_{s}+1\right)} d N_{s} .
\end{aligned}
$$

$\log 0$

$$
\begin{aligned}
\operatorname{Var}\left(\widehat{A}_{\ell}\right)= & \int_{0}^{t} \frac{n \exp \left\{-\widehat{v} s^{\widehat{b}}\right\} \widehat{v} \widehat{b} s^{\hat{b}-1}}{\left(n \exp \left\{-\widehat{v} s^{\hat{b}}\right\} d s+Y_{s}+1\right)\left(n \exp \left\{-\widehat{v} s^{\hat{b}}\right\}+Y_{s}\right)} \\
& +\int_{0}^{t} \frac{\left(n \exp \left\{-\widehat{v} s^{\widehat{b}}\right\}+Y_{s}-1\right)}{\left(n \exp \left\{-\widehat{v} s^{\widehat{b}}\right\}+Y_{s}\right)^{2}\left(n \exp \left\{-\widehat{v} s^{\widehat{b}}\right\}+Y_{s}+1\right)} d N_{s} .
\end{aligned}
$$

onde $v-1 / a^{b}$. A tabela 5.3 apresenta as estimativas da variância do estimador Bayesiano. 


\begin{tabular}{|c|c|c|c|}
\hline tempos & variância & tempos & variância \\
\hline \hline 19 & 0.000006 & 349 & 0.445029 \\
\hline 43 & 0.015270 & 398 & 0.5139938 \\
\hline 44 & 0.016049 & 477 & 0.612978 \\
\hline 148 & 0.137069 & 514 & 0.6153615 \\
\hline 169 & 0.167770 & 595 & 0.729415 \\
\hline 171 & 0.170754 & 603 & 0.735929 \\
\hline 205 & 0.222701 & 662 & 0.778753 \\
\hline 232 & 0.265045 & 700 & 0.801861 \\
\hline 248 & 0.290313 & 701 & 0.802419 \\
\hline 250 & 0.2933469 & 706 & 0.805126 \\
\hline 263 & 0.313941 & 709 & 0.806685 \\
\hline 271 & 0.326492 & 710 & 0.807181 \\
\hline 282 & 0.343659 & 763 & 0.829560 \\
\hline 290 & 0.356061 & 777 & 0.834200 \\
\hline 347 & 0.442106 & 869 & 0.853104 \\
\hline
\end{tabular}

Tabela 4.1: Estimativas da variância da taxa de falha acumulada via estimador Bayesiano

Faremos agora a aplicação do estimador de Bayes 4.3 aos dados dos freios. A T'abela 4.2 apresenta as estimativas para a taxa de falha, taxa de falha acumulada e função de confiabilidade baseadas no estimador Bayesiano não-paramétrico, calculadas conforme o procedimento da Seção 1.1 [veja Equaçäo 1.6]. Apresentamos também, os gráficos com as estimativas para a taxa de falha, taxa de falha acumulada e funçāo de confiabilidade calculados via Kaplan-Meier, Nelson-Aalen, Weibull e Bayesiano não-paramétrico. 


\begin{tabular}{|c|c|c|c|c|}
\hline Tempros & Tiaxa de Fiallata & laxide & - lablha Acumulada & Confiabilidade \\
\hline $1 ! 9$ & $0.011: 721$ & & 0.01913016 & 0.9 and \\
\hline 4.3 & $0.0171: 16$ & & 0.011801 & 0. 45 मी1.31 \\
\hline 44 & $0.01-153$ & & $0.062-26$ & 0.439201 \\
\hline 1.18 & $(1.019041$ & & $0.0 \times$ sitio $_{0}$ & 0.915157 \\
\hline 169 & $0.019-8.8$ & & 0.127118 & $0.8 \sin 20$ \\
\hline $1-1$ & $(1+t) 2 f) 21+1$ & & (1.5364-1) & $0 . \overline{1}+2 \times 24 \bar{i}$ \\
\hline 267 & $0.172131=$ & & 0.385252 & 0.625509 \\
\hline 232 & (1.02) $215 \mathrm{~s}$ & & 0.13795 & 0.14535 \\
\hline $21 x$ & (1.0233:1:) & & $(1.170609)$ & 0.105094 \\
\hline 250 & $0.024016 \mathrm{i}$ & & 0.506024 & 0.602888 \\
\hline 263 & $0.0250 \times 11$ & & (1.5.16453 & ו1ו \\
\hline 27 & $0 .(132,564$ & & $0,5 \times 3 n 18$ & $0.55 \times 211$ \\
\hline 282 & 0.026981 & & 0.621192 & 0.5355333 \\
\hline 2011 & $0.012 \times(1 ; 31$ & & (1.64t2).14s & 0.51507 .2 \\
\hline 3.7 & 0.031310 & & 0.776873 & 0.45942 \\
\hline 319 & 0.032418 & & 0.81227 & $0.4+3841 ;$ \\
\hline 398 & (1.03tilat & & (i.stidefix & (1.3964125 \\
\hline . $17 \pi$ & 0.0426333 & & 1.0953988 & $(1.33 .14063$ \\
\hline 511 & $(1.018: 0101$ & & 1.203102 & 0.300361 \\
\hline 595 & (1.05ti5y:3 & & 1.300725 & 0.215895 \\
\hline $60: 3$ & 0.06076656 & & 1.4t+1406 & 0.231216 \\
\hline $6+62$ & $(1,0-1) 28$ & & 1.63253 & $0.195 .1 \times 9$ \\
\hline $7(3)$ & $0.0 \times 150$ & & 1.753 & $0.1+5 \leqslant 9+27$ \\
\hline 701 & $0.08 x+161$ & & 1.26 .5968 & 0.151746 \\
\hline $70 \%$ & o.ogkinge & & 1.973660 & 0.1389333 \\
\hline 709 & 0.110012 & & 2.1089853 & $0.1 \geq 3705$ \\
\hline (ii) & $0.123 \mathrm{BS}$ & & 2.216959 & 0.100407 \\
\hline$\pi(13$ & 0.161613 & & 2.511446 & 0.0811 .31 \\
\hline$\pi$ & 0.206:11 & & 2.752521 & (1.06534) \\
\hline$x_{1} s_{4}$ & 0.345930 & & 3.118700 & $0.018-3=5$ \\
\hline
\end{tabular}

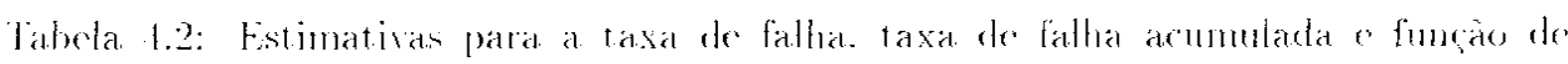
contiabilidado baseadas no estimador Bayesiduo mào-patramétrico 


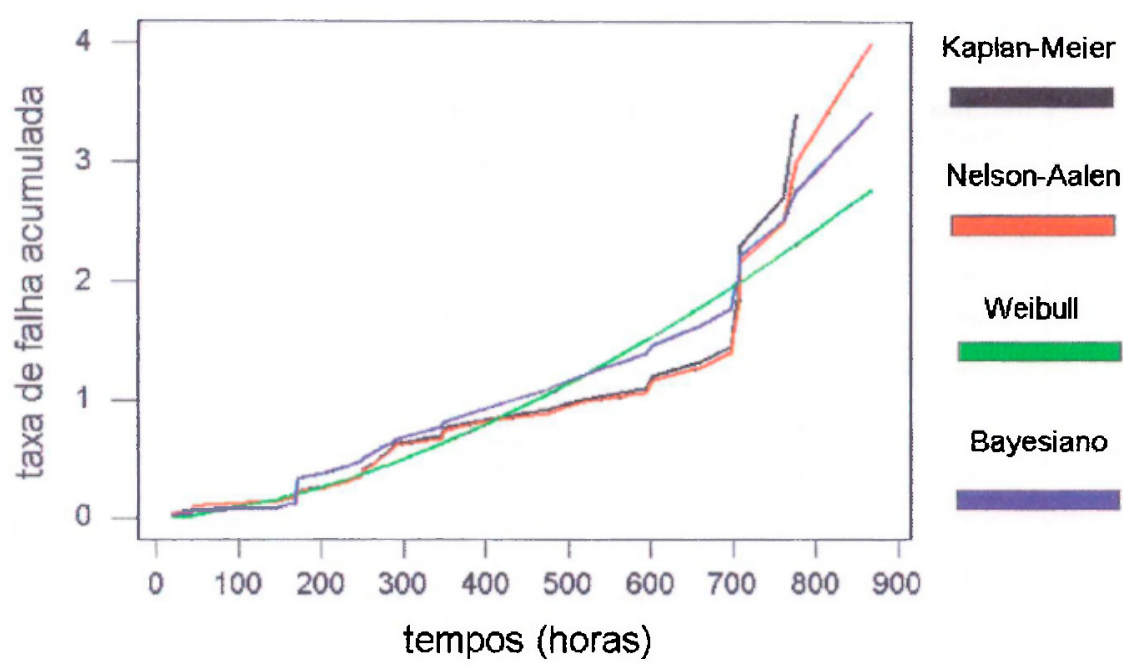

Figura 4.1: Taxa de Falha Acumulada

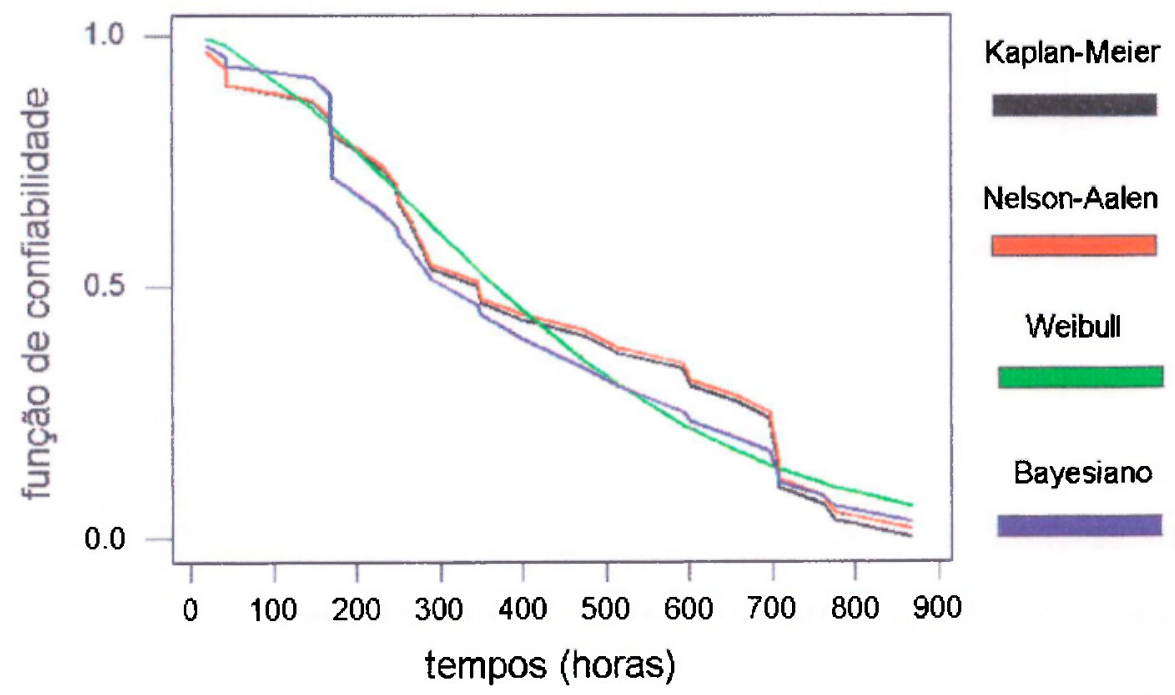

Figura 4.2: Função de Confiabilidade 


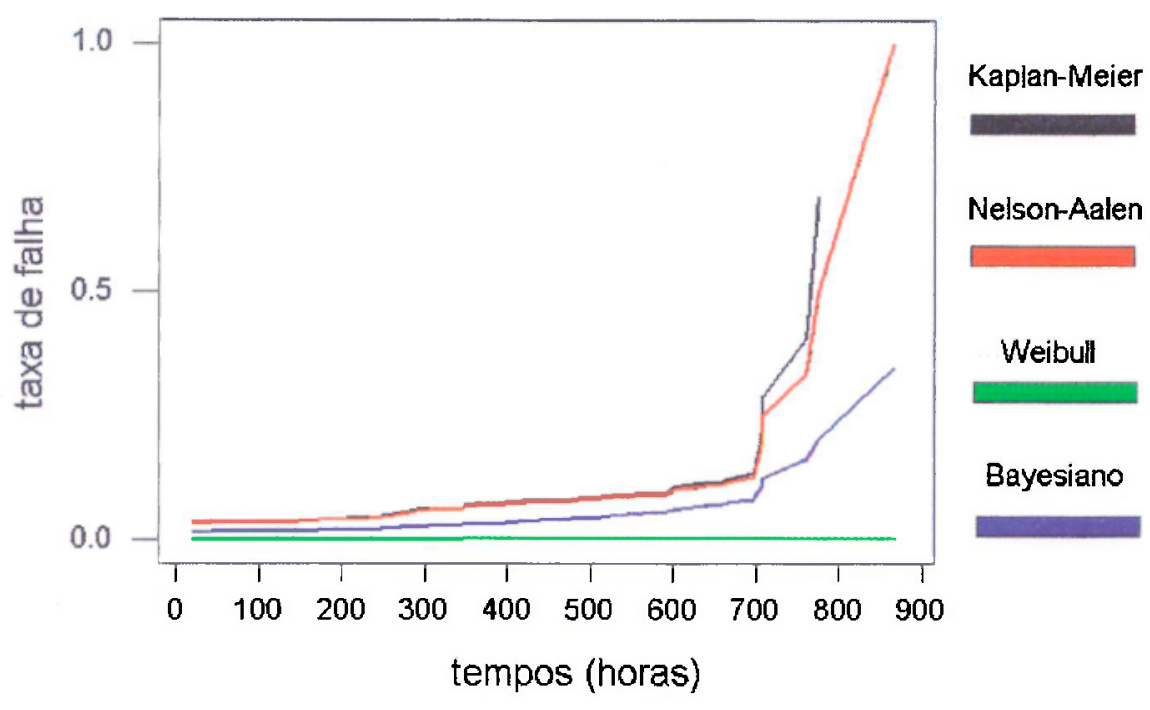

Figura 4.3: Taxa de Falha 


\section{Capítulo 5}

\section{Considerações Finais}

\subsection{Conclusões}

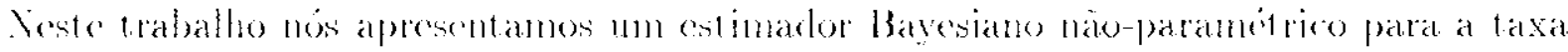

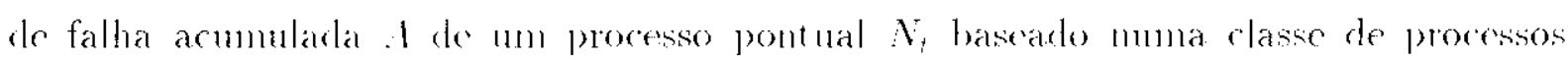
de: Iery puro saltu chamados de processos beta [Hjort (1990). Kim (1999)] c também sugerimos $11 m$ estimador para a lava de fallad $\lambda$. Kim a Iar (2001) mostraram que a

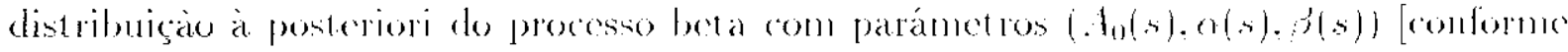

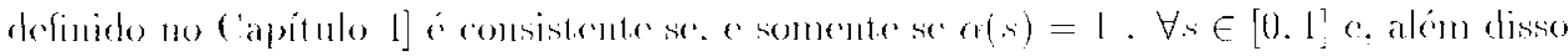

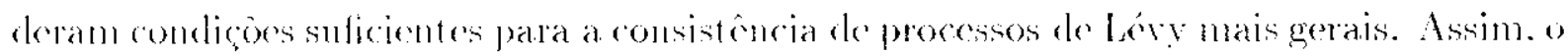
processo beta detinido por lljort. processos gama processos de l)irichlet lem distribuicào à posteriori consistente. Jates estimadores apmesentaram desempenho próximo an dos estimadores de kaplan-Meror e Velson-Aalen. conforme os grálicos c tabelas apresentadon mos apilulos anderiones.

Por excmplo, observe na Figuta 5.1 come os gráficos da taxa do falla acumulada estimada via estimadores de Kaplan-Meice r Nelson-Aalen săo acompanhados pelo estimador Bayesialuo. 


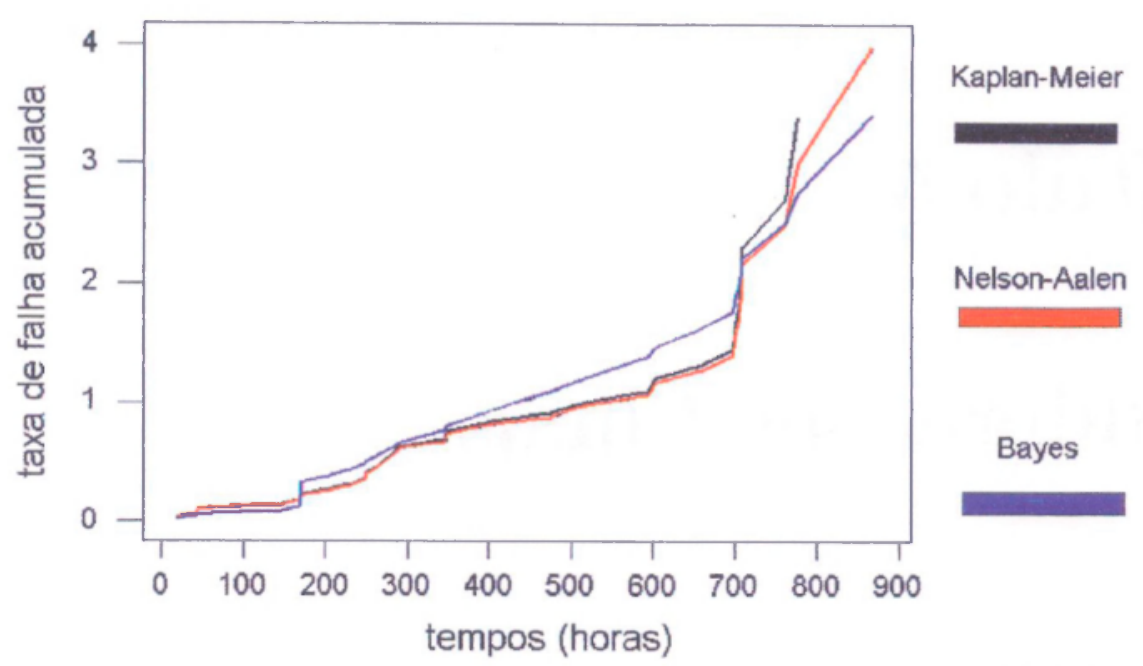

Figura 5.1: Taxa de Falha Acumulada

Isso não surpreende quando observamos o comportamento desse estimador,

$$
\widehat{A}(t)=\int_{0}^{t} \frac{\beta(s) \lambda(s) d s}{\beta(s)+Y(s)}+\int_{0}^{t} \frac{d N(s)}{\beta(s)+Y(s)},
$$

se a função $\beta(s) \rightarrow 0$, o estimador de Bayes converge para o estimador de Nelson-Aalen e se $\beta(s) \rightarrow \infty$ obtemos a taxa acumulada $A_{0}$ dada como priori. No caso do estimador para a taxa de falha,

$$
\hat{\lambda}(s)=\frac{\beta(s) \lambda_{0}(s)}{\beta(s)+Y(s)}+\frac{1}{\beta(s)+Y(s)}
$$

observamos que se $\beta(s) \rightarrow 0$, então $\widehat{\lambda}$ coincide com o estimador proposto por Kaplan-Meier [ver Capítulo 1]. Assim, uma $\beta(s)$ apropriada pode levar a melhores resultados.

As tabelas 5.1, 5.2 e 5.3 apresentam as estimativas da variância dos estimadores da taxa de falha acumulada, baseados nos estimadores de Kaplan-Meier, Nelson-Aalen e Bayesiano. Observe nas tabelas que o estimador

$$
\widehat{A}(t)=\int_{0}^{t} \frac{n \exp \left\{-\widehat{v} s^{\hat{b}}\right\} \widehat{v} \hat{b} s^{\hat{b}-1}}{n \exp \left\{-\widehat{v} s^{\hat{b}}\right\}+Y(s)} d s+\int_{0}^{t} \frac{d N_{s}}{n \exp \left\{-\widehat{v} s^{\hat{b}}\right\}+Y(s)},
$$

possui variância maior que os estimadores empíricos. 


\begin{tabular}{|c|c||c|c|}
\hline tempos & variância & tempos & variância \\
\hline \hline 19 & 0.001149 & 349 & 0.038095 \\
\hline 43 & 0.002381 & 398 & 0.043590 \\
\hline 44 & 0.003704 & 477 & 0.050000 \\
\hline 1.18 & 0.005128 & 514 & 0.057576 \\
\hline 169 & 0.006667 & 595 & 0.0666667 \\
\hline 171 & 0.008333 & 603 & 0.077778 \\
\hline 205 & 0.010145 & 662 & 0.091667 \\
\hline 232 & 0.012121 & 700 & 0.109524 \\
\hline 248 & 0.014286 & 701 & 0.133333 \\
\hline 250 & 0.016667 & 706 & 0.1666667 \\
\hline 263 & 0.019298 & 709 & 0.216667 \\
\hline 271 & 0.022222 & 710 & 0.300000 \\
\hline 282 & 0.025490 & 763 & 0.466667 \\
\hline 290 & 0.029167 & 777 & 0.966667 \\
\hline 347 & 0.033333 & 869 & $*$ \\
\hline
\end{tabular}

Tabela 5.1: Estimativas da variância da taxa de falha acumulada via Kaplan-Meier

\begin{tabular}{|c|c|c|c|}
\hline tempos & variância & tempos & variância \\
\hline 19 & 0.00111 & 349 & 0.03615 \\
\hline 43 & 0.00230 & 398 & 0.04126 \\
\hline 44 & 0.00358 & 477 & 0.04717 \\
\hline 148 & 0.00495 & 514 & 0.05412 \\
\hline 169 & 0.00643 & 595 & 0.06238 \\
\hline 171 & 0.00803 & 603 & 0.07238 \\
\hline 205 & 0.00976 & 662 & 0.08473 \\
\hline 232 & 0.01165 & 700 & 0.10035 \\
\hline 248 & 0.01372 & 701 & 0.12076 \\
\hline 250 & 0.01599 & 706 & 0.14854 \\
\hline 263 & 0.01849 & 709 & 0.18854 \\
\hline 271 & 0.02126 & 710 & 0.25104 \\
\hline 282 & 0.02434 & 763 & 0.36215 \\
\hline 290 & 0.02780 & 777 & 0.61215 \\
\hline 347 & 0.03171 & 869 & 1.61215 \\
\hline
\end{tabular}

Tabela 5.2: Estimativas da variância da taxa de falha acumulada via Nelson-Aalen 


\begin{tabular}{|c|c|c|c|}
\hline tempos & variância & tempos & variância \\
\hline \hline 19 & 0.000006 & 349 & 0.445029 \\
\hline 43 & 0.015270 & 398 & 0.513938 \\
\hline 44 & 0.016049 & 477 & 0.612978 \\
\hline 148 & 0.137069 & 514 & 0.653615 \\
\hline 169 & 0.167770 & 595 & 0.729415 \\
\hline 171 & 0.170754 & 603 & 0.735929 \\
\hline 205 & 0.222701 & 662 & 0.778753 \\
\hline 232 & 0.265045 & 700 & 0.801861 \\
\hline 248 & 0.290313 & 701 & 0.802419 \\
\hline 250 & 0.293469 & 706 & 0.805126 \\
\hline 263 & 0.313941 & 709 & 0.806685 \\
\hline 271 & 0.326492 & 710 & 0.807181 \\
\hline 282 & 0.343659 & 763 & 0.829560 \\
\hline 290 & 0.356061 & 777 & 0.834200 \\
\hline 347 & 0.442106 & 869 & 0.853104 \\
\hline
\end{tabular}

Tabela 5.3: Estimativas da variância da taxa de falha acumulada via estimador Bayesiano

Na Figura 5.2 apresentamos os gráficos da taxa de falha estimada via estimador de Nelson-Aalen e o estimador proposto 5.2 para a taxa de falha. Nota-se no gráfico que até 700 horas, a estimativa da taxa de falla via estimador 5.2 acompanha bem o grálico da taxa de falha estimada via Nelson-Aalen. Esses resultados mostram a flexibilidade do estimador Bayesiano não-paramétrico para modelagem de dados de confiabilidade, apresentando-se como uma alternativa aos modelos existentes.

Embora tenhamos apresentado o estimador baseado cm dados não censurados, podemos admitir dados com diferentes tipos de censuras conforme exemplos de Kim (1999) e além disso, o processo à priori $A$ pode apresentar tempos de descontinuidades fixados [ver Apêndice]. 


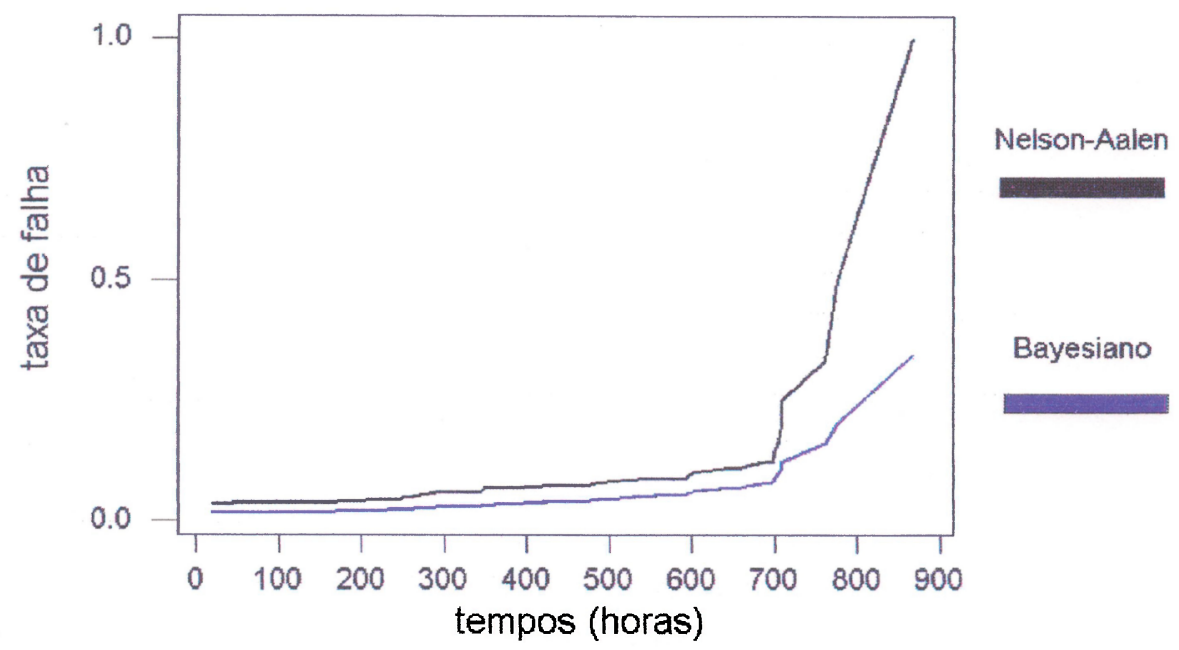

Figura 5.2: Taxa de Falha

\subsection{Propostas Futuras}

Propomos estudar os seguintes tópicos que não foram desenvolvidos durante esse trabalho, embora haja :

a) Propriedades assintóticas dos estimadores obtidos da taxa de falha e da taxa de falha acumulada;

b) simulações dos Processos de Lévy. 


\section{Capítulo 6}

\section{Apêndice}

\subsection{Espaço de Cantor}

O espaço de Cantor é um espaço formado pelo produto cartesiano de infinitas cópias de $S=\{0,1\}$

$$
S^{\infty}=S \times S \times \times S \ldots
$$

Munido da topologia discreta ele é um espaço compacto pois cada $S$ é compacto (teorema de Tychonoff).

A projeção coordenada é uma aplicação

$$
\pi: S^{\infty} \longrightarrow S
$$

dada por

$$
\pi_{k}(w)=w_{k}, k \in \mathbb{N}
$$

e $w=\left(w_{1}, w_{2}, w_{3} \ldots\right) \in S^{\infty}$.

Fssas projeçōes geram uma classe $\mathcal{H}$ definida da seguinte forma

$$
\mathcal{H}=\left\{\pi_{k}^{-1}\left(\left\{w_{k}\right\}\right): w_{k} \in S, k \in \mathbb{N}\right\} .
$$

Desta definição de $\mathcal{H}$ temos os seguintes resultados diretos:
(1) $\pi_{k}^{-1}(\{1\}) \cup \pi_{k}^{-1}(\{0\})=S^{\infty}$
(2) $\pi_{k}^{-1}(\{1\}) \cap \pi_{k}^{-1}(\{0\})=\varnothing$. 
Lema 6.1.1. A classe $\mathcal{H}$ separa pontos em $S^{\infty}$, isto é, dados $x$ e y pontos distintos de $S^{\infty}$ existe um elemento $\triangle$ de $\mathcal{H}$ tal que

$$
I_{\{\Delta\}}(x) \neq I_{\{\Delta\}}(y)
$$

Dem: Tomemos dois clementos x e y em $S^{\infty}$.

$$
\begin{array}{ll}
x=\left(\alpha_{1}, \alpha_{2}, \alpha_{3}, \ldots, \alpha_{k}, \ldots\right), & \alpha_{i}=0 \text { ou } 1 \\
y=\left(\beta_{1}, \beta_{2}, \beta_{3}, \ldots, \beta_{k}, \ldots\right), & \beta_{i}=0 \text { ou } 1
\end{array}
$$

distintos, isto é, x e y são diferentes em pelo menos uma coordenada, digamos na k-ésima coordenada, assim $\alpha_{k} \neq \beta_{k}$. Consideremos as imagens inversas

$$
\pi_{k}^{-1}\left(\left\{\alpha_{k}\right\}\right) \text { e } \pi_{k}^{-1}\left(\left\{\beta_{k}\right\}\right)
$$

pelo resultado anterior (2) temos

$$
\pi_{k}^{-1}\left(\left\{\alpha_{k}\right\}\right) \cap \pi_{k}^{-1}\left(\left\{\beta_{k}\right\}\right)=\varnothing
$$

dessa forma, basta tomarmos $\Delta=\pi_{k}^{-1}\left(\left\{\alpha_{k}\right\}\right)$ para termos

$$
I_{\{\Delta\}}(x)=1 \neq I_{\{\Delta\}}(y)=0
$$

Lema 6.1.2. A classe $\mathcal{H}$ de subconjuntos de $S^{\infty}$ é compacta, isto é, para toda sequência $\left\{C_{n}\right\} \subset \mathcal{H}$ satisfazendo $\bigcap_{n=1}^{\infty} C_{n}=\varnothing$, existe um $n_{0} \in \mathbb{N}$ que satisfaz $\bigcap_{n=1}^{n_{0}} C_{n}=\varnothing$.

Dem:

Considere a sequência $\left\{C_{n}\right\} \subset \mathcal{H} \operatorname{com} \bigcap_{n=1}^{\infty} C_{n}=\varnothing$. Nesse caso existem $\mathrm{m}$ en $\in \mathbb{N}$ tais que

$$
C_{m}=\pi_{k}^{-1}(\{1\}) \quad \text { c } \quad C_{n}=\pi_{k}^{-1}(\{0\})
$$

para algum $k \in \mathbb{N}$. Se nós tomarmos $n_{0}=\max \{m, n\}$, teremos $\bigcap_{n=1}^{n_{0}} C_{n}=\varnothing$.

Vamos considerar agora uma classe formada por intersecçóes finitas de elementos de $\mathcal{H}$

$$
\Delta=\left\{A \subset S^{\infty}: A=B_{1} \cap B_{2} \cap B_{3} \cap \ldots \cap B_{n}, \quad B_{i} \in \mathcal{H}, \quad n \in \mathbb{N}\right\} \cup\{\varnothing\} \cup\left\{S^{\infty}\right\}
$$

Lema 6.1.3. A classe $\Delta$ acima é uma semi-álgebra. 
Considere agora a classe $\mathcal{D}$ de todos os subconjuntos finitos de números naturais

$$
\mathcal{D}=\left\{\left(v_{1}, v_{2}, \ldots, v_{n}\right): v_{i} \in \mathbb{N}, i=1,2, \ldots, n\right\}
$$

Lema 6.1.4. A classe $\Delta$ anterior pode ser escrita como

$\Delta=\left\{\pi_{v_{1}}^{-1}\left(\left\{w_{v_{1}}\right\}\right) \cap \ldots \cap \pi_{v_{n}}^{-1}\left(\left\{w_{v_{n}}\right\}\right):\left(v_{1}, \ldots, v_{n}\right) \in \mathcal{D},\left(w_{v_{1}}, \ldots, w_{v_{n}}\right) \in S^{n}, n \in \mathbb{N}\right\} \cup\{\varnothing\} \cup\left\{S^{\infty}\right\}$

Lema 6.1.5. A álgebra $\mathcal{A}$ gerada pela classe $\Delta$ é dada por

$\mathcal{A}=\left\{A \subset S^{\infty}: A=B_{1} \cup B_{2} \cup \ldots \cup B_{n}, \quad B_{i} \in \Delta, i=1, \ldots, n, B_{i} \cap B_{j}=\varnothing(i \neq j), n \in \mathbb{N}\right\}$

Vamos denotar por $\mathcal{G}$ a $\sigma$-álgebra gerada por $\mathcal{A}$.

\subsection{Alguns Resultados Utilizados}

Definição 6.2.1. Uma função $f$, definida em um espaço mensurável $X$ é chamada simples se existe uma classe finita disjunta $\left\{E_{1}, \ldots, E_{n}\right\}$ de conjuntos mensuráveis e um conjunto finito $\left\{\alpha_{1}, \ldots, \alpha_{n}\right\}$ de números reais tais que

$$
\varphi_{n}(x)=\sum_{i=1}^{n} \alpha_{i}^{n} I_{E_{i}^{n}}(x)
$$

Teorema 6.2.1. Seja $f$ uma função mensurável não negativa. Então existe uma sequência $\left\langle\varphi_{n}\right\rangle$ de funções mensuráveis simples tais que, para cada $x, \varphi_{n}(x) \rightarrow f(x)$.

Definição 6.2.2. Um tempo $t \in \mathbb{R}_{+}$é chamado um tempo de descontinuidade fixado para um processo com incrementos independentes $X$ se $P\left[\Delta X_{t} \neq 0\right]>0$.

O conjunto dos tempos de descontinuidade fixados é no máximo contável (esta propriedade é verdadeira para processos càdlàg). Processos com incrementos independentes estacionários não possiem tempos de descontinuidade fixados. 


\section{Referências Bibliográficas}

[1] O. Aalen (1978) - Nonparametric Inference for a Family of Counting Processes, Ann. Statist., 6,pg. 701-726.

[2] J. I. Ansell e M. J. Phillips (1994) - Practical Methods for Reliability Data Analysis, Oxford Science Publications, New York.

[3] D. P. Bertsekas e S. P. Shireve (1978) - Stochastic Optimal Control: The Discrete Time Case, Academic Press, New York.

[4] P. Billingsley (1968) - Convergence of Probability Measures, John Wiley and Sons, New York.

[5] K. L. Chung (1974) - A course in Probability Theory, Ed. 2, Academic Press, New York.

[6] P. Damien, P. W. Laud e A. F. M. Smith, (1995) - Random Variate Generation from Infinitely Divisible Distributions with Applications to Bayesian Inference, J. R. Statist. Soc. B, 57, pg. 547-564.

[7] K. Doksum (1974) - Tailfree and Neutral Random Probabilities an Their Posterior Distributions, Ann. Probability, 2, pg. 183-201.

[8] R. L. Dykstra e Purushottam Laud (1981) - A Bayesian Nonparametric Approach to Reliability, Ann. Statist., 9, pg. 357-367.

[9] T. R. Flemimg e D. P. IIarrington (1990) - Counting Processes and Survival Analisys, John Wiley and Sons, New York.

[10] T. S. Ferguson e M. J. Klass (1972) - A Representation of Independent Increment Processes Without Gassian Components, Ann. of Math. Statist., V. 43,5, pg. 16341643. 
[11] T. S. Ferguson (1973) - A Bayesian Analysis of Some Nonparametric Problems, Ann. Statist., 1, pg. 209-230.

[12] T. S. Ferguson (1974) - Prior Distributions on Spaces of Probability Measures, Ann. Statist., 2, pg. 615-629.

[13] T. S. Ferguson e E. G. Phadia (1979) - Bayesian Nonparametric Estimation Based on Censored Data, Ann. Statist., 7, pg. 163-186.

[14] N. L. Hjort (1990) - Nonparametric Bayes Estimators Based on Beta Processes in Models for Life History Data, Ann. Stat., 18, N.3 pg. 1259-1294.

[15] J. Jacod (1979) - Calcul Stochastique et Problèmes de Martingales, Lectures Notes in Mathematics,Springer-Verlag, Berlin, 714

[16] J. Jacod e A.N. Shiryaev (1987) - Limit Theorems for Stochastic Processes, SpringerVerlag, New York.

[17] E. L. Kaplan e P. Meier (1958) - Nonparametric Estimation from Incomplete Observations, American Statistical Association Journal, 53, pg. 457-481.

[18] Y. Kim (1999) - Nonparametric Bayesian Estimators for Counting Processes, Ann. Stat. , 27, N.2, pg. 589-599.

[19] Y. Kim e J. Lee (2001) - On Posterior Consistency of Survival Models, Ann. Stat., 29, N.3, pg. 666-686.

[20] A. N. Kolmogorov e S. V. Fomin (1970) - Introductory Real Analysis, Dover Publications, New York.

[21] J. P. Klein (1991) - Small Sample Moments of Some Estimators of the Variance of the Kaplan-Meier and Nelson-Aalen Estimators, Scand. J. Statistics, 18, pg. 333-340.

[22] D. Leão, M. D. Fragoso and P. Ruffino (1999) - Characterization of Radon spaces, Probability and Statistical Letters, 42,pg. 409-413.

[23] D. Leão, M. D. Fragoso and P. Ruffino (2001) - Regular Conditional Probability, Desintegration of Probability and Radon Spaces, Submitted Annals of Probability.

[24] R. S. Liptser e A. N. Shiryayev (1977) - Statistics of Random Processes I: General Theory, Springer-Verlag, New York. 
[25] R. S. Liptser e A. N. Shiryayev (1978) - Statistics of Random Processes II: General Theory, Springer-Verlag, New York.

[26] Jacod, J. e Shiryaev, A. N. (1987) - Limit Theorems for Stochastic Processes, Springer-Verlag, New York.

[27] E. Marczewski (1953) - On compact measures, Fundamentae Mathematica, 40, pg. 409-413.

[28] M. Métivier (1982) - Semimartingales: A course in Stochastic Processes, de Gruyter Studies in Mathematics, 2.

[29] P. Meyer (1966) - Probabilités et Potentiel (Lère édition), Hermann, Paris.

[30] W. Nelson (1972) - Theory and Applications of Hazard Plotting for Censored Failure Data, Technometrics ,42, N.1.

[31] J. Neveu (1965) - Mathematical Foundations of The Calculus of Probability, HoldenDay,Inc, San Francisco, California.

[32] W. J. Padget e L. J. Wei (1981) - A Bayesian Nonparametric Estimator of Survival Probability Assuming Increasing Failure Rate, Comm. Statist. Theory Methods 10, pg. 49-63.

[33] B. L. S. Prakasa Rao (1987) - Asymptotic Theory of Statistical Inference, 21, Wiley, New York.

[34] P. Protter (1990) - Stochastic Integration and Differential Equations: a new approach, Applications of Mathematics, 21, Springer-Verlag, Berlin.

[35] S. G. Walker e P. Damien, (1998) - A full Bayesian Nonparametric Analysis Involving a Neutral to the Right processes, Scand. J. Statist. ,25, pg. 669-680.

[36] S. G. Walker e P. Damien, (2000) - Representations of Lévy processes without Gaussian components, Biometrika,87, 2, pg. 477-483.

[37] R. L. Wolpert e K. Ickstadt, (1998) - Gamma/Poisson Random Field Models for Spatial Statistics, Biometrika, 85, pg. 251-267. 